\title{
Teoria da Confiabilidade em um Modelo de Tempo de Vida Geral: Importância de Componentes Burn-in
}

José Elmo de Menezes

\author{
Tese apresentada \\ ao \\ Instituto de Matemática e Estatística \\ $d a$ \\ Universidade de São Paulo \\ para \\ obtenção do grau \\ de \\ Doutor em Estatística \\ Área de Concentração: Probabilidades \\ Orientador: Prof. Dr. VANDERLei da Costa Bueno
}

Este trabalho contou com apoio financeiro do CNPq, Processo № 141815/2002-7.

São Paulo - Agosto - 2005 


\section{Teoria da Confiabilidade em um Modelo de Tempo de Vida Geral: Importância de Componentes e Burn-in}

Este exemplar corresponde à redação final da tese devidamente corrigida e defendida por José Elmo de Menezes e aprovada pela comissão julgadora.

São Paulo, 12 de agosto de 2005.

Banca examinadora:

- Prof. Dr. Vanderlei da Costa Bueno (Orientador) - IME -USP

- Prof. Dr. Nelson Ithiro Tanaka - IME -USP

- Prof. Dr. João Batista Camargo Júnior - EP -USP

- Prof. Dr. Oswaldo Luiz do Valle Costa - EP -USP

- Prof. Dr. Mauro Sérgio de Freitas Marques - UNICAMP 


\section{Epígrafe}

\section{Tocando em Frente}

Ando devagar porque já tive pressa e levo esse sorriso porque já chorei demais. Hoje me sinto mais forte, mais feliz, quem sabe, eu só levo a certeza de que muito pouco sei, ou nada sei.

Conhecer as manhas e as manhãs, o sabor das massas e das maçãs.

É preciso amor prá poder pulsar, é preciso paz prá poder sorrir, é preciso chuva para florir.

Penso que cumprir a vida seja simplesmente compreender a marcha e ir tocando em frente.

Como um velho boiadeiro levando a boiada eu vou tocando os dias pela longa estrada, estrada eu sou, estrada eu vou.

Todo mundo ama um dia, todo mundo chora, uma dia a gente chega e no outro vai embora.

Cada um de nós compõe a sua propria história e cada ser em si carrega o dom de ser capaz de ser feliz.

Almir Sater e Renato Teixeira 


\section{Agradecimentos}

- A Deus, pelo dom da vida.

- Ao professor Vanderlei Bueno, pela orientação e amizade.

- A minha esposa Cristina e meu filho Gabriel com amor e um pedido de desculpa pelos momentos ausentes.

- Aos meus pais, Carlos e Nair, os grandes responsáveis pela minha existência.

- Aos meus irmãos, Flora, Aloisio, Egidio, Antônio, Cida e Luciano.

- Aos amigos da extinta sala 140, bloco B do IME-USP e adjacentes, Barbara, Domingo, Edijane, Joelmir, Jocirei, Iran, Mauricio, Nelson, Rogério, Ronaldo, Ruldimar, Samuel, Ulisses e Willian pela amizade e as conversas acadêmicas e não acadêmicas.

- Aos professores do IME-USP.

- Aos funcionários da secretaria e do cafezinho (copa) do IME.

- Aos colegas do MAF-UCG, pela amizade e apoio a minha saída para o doutorado, em especial aos professores, Cristian, Ovídio, Pedrão e Vinício e ao nosso diretor Nilton Olimpio.

- A todos aqueles que com probabilidade um foram esquecidos, mas foram personagens essenciais na minha trajetória, meu muito obrigado. 
A minha esposa

Cristina e meu filho

Gabriel, com amor de esposo e pai. 


\section{Abstract}

In this work we analyze three main topics that generalize some classical results on Reliability Theory, all of them associated with a general lifetime model. Firstly, we generalize the Barlow and Proschan concept on component's reliability importance for system reliability under dependence conditions. In a second part, we observe the system at component's levels and define a measure of reliability importance through the innovations gain processes, generalizing a result from Norros (1986b). Finally, we analyze Burn-in models involving rewards and costs processes in order to determine the optimal stopping time, based on a the ILA-stopping rule (infinitesimal-lookahead). 


\section{Resumo}

Neste trabalho abordamos três temas principais que generalizam alguns resultados clássicos da Teoria da Confiabilidade, todos associados a um modelo de tempo de vida geral. Na primeira parte estudamos o conceito da importância da confiabilidade de um componente para a confiabilidade do sistema de Barlow e Proschan. Na segunda parte, observando o sistema a níveis de seus componentes, definimos uma medida de importância através dos processos de ganhos de inovações, generalizando um resultado de Norros (1986b). Finalizamos este trabalho analisando modelos de Burn-in envolvendo processos de custos e ganhos e encontrando um tempo de parada ótimo baseado na regra de parada IOPF ( infinitesimal olhada para frente). 


\section{Sumário}

Introdução

1 Medidas de Importância $\quad 6$

1.1 Introdução . . . . . . . . . . . . . . . . . . . 6

1.2 Medida de Importância para Componentes Independentes . . . . . . 7

1.3 Medida de Importância para Blocos . . . . . . . . . . . . . . . 10

1.3.1 Processos Pontuais Marcados . . . . . . . . . . . . . . . . 10

1.3.2 Medida de Importância através dos Compensadores . . . . . 16

1.4 Comparando medidas de importâncias através de transformações nos

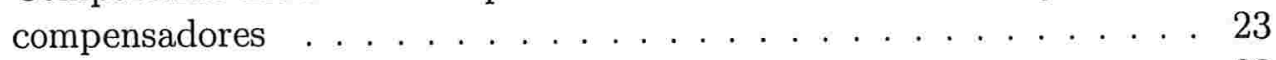

1.5 Importância de um Módulo . . . . . . . . . . . . . . 33

2 Importância em um Sistema Modelado por uma Cadeia de Markov $\begin{array}{ll}\text { não homogênea } & 39\end{array}$

2.1 Introdução . . . . . . . . . . . . . . . . . . . 39

2.2 Formulação Matemática . . . . . . . . . . . . . . . . . . . . . . 40

2.3 Importância do Componente . . . . . . . . . . . . . . . . . . 45

2.4 Aplicação: O Caso para $N=2$ Estados . . . . . . . . . . . . . 50

3 Um modelo de Burn-in $\quad \mathbf{5 6}$

3.1 Introdução . . . . . . . . . . . . . . . . . 56

3.2 Formulação Matemática . . . . . . . . . . . . . . . . . 57

3.3 Soluções para os Problemas . . . . . . . . . . . . . . . . . 6 63

3.3.1 Uma Solução para o Problema $1 \ldots$. . . . . . . . . . . . . . . 63

3.3.2 Uma Solução para o Problema 2 . . . . . . . . . . . . . . . . 68

$\begin{array}{ll}\text { A Algumas Definições } & 78\end{array}$

A.1 Modelos Estocásticos de Falhas . . . . . . . . . . . . . . . . . 78

A.2 Definições em Confiabilidade . . . . . . . . . . . . . . . . 93

$\begin{array}{ll}\text { Bibliografia } & 98\end{array}$ 


\section{Introdução}

Na Teoria de Confiabilidade, a análise de sistemas complexos com componentes dependentes é uma área de pesquisa em desenvolvimento, que começou com os trabalhos de Arjas (1981 e 1989), Jensen (1989 e 1990 ), Norros(1986a e 1986b) e outros. Um livro recente que trata a Teoria de Confiabidade com tal abordagem e resume os resultados alcançados é o de Aven, e Jensen, (1998).

Para analisar a dependência entre os componentes e a dependência estrutural entre o sistema e seus componentes na dinâmica do tempo, vários autores utilizam a teoria dos martingais para processos pontuais e quase sempre definem como modelo geral o semimartingal regular (SMR),

$$
I(T \leq t)=\int_{0}^{t \wedge T} \lambda_{s} d s+M_{t}
$$

onde $T$ é o tempo de vida do sistema, $\lambda=\left(\lambda_{s}\right)_{s \geq 0}, s \in \mathbb{R}_{+}$é um processo estocástico progressivamente mensurável, com $E\left[\int_{0}^{t} \lambda_{s} d s\right]<\infty$, para todo $t \in \mathbb{R}_{+}$, e $M=$ $\left(M_{s}\right)_{s \geq 0}$ é um martingal uniformemente integrável de média zero, com respeito à uma família de sub- $\sigma$-álgebra de $\Im, \mathbf{F}=\left(\Im_{s}\right)_{s \geq 0}$ onde $\Im_{s}$ representa a informação até o instante $s$.

Desde que $I(T \leq t)$ seja um submartingal com respeito a $\Im_{t}$, podemos interpretar a relação (1) como a decomposição de Doob-Meyer

$$
M_{t}=I(T \leq t)-\int_{0}^{t \wedge T} \lambda_{s} d s
$$


que caracteriza completamente a intensidade $\lambda_{s}$ pois o compensador

$$
A(t)=\int_{0}^{t \wedge T} \lambda_{s} d s
$$

é contínuo em $t$ e portanto previsível.

Em geral, na teoria clássica observa-se $\Im_{t}=\sigma\{I(T>s), s \leq t\}$ e a intensidade de falha do sistema $\lambda_{s}$ é justamente a sua taxa de falha no conjunto $\{T>s\}$, isto é, $\lambda_{s}=\frac{f(s)}{\bar{F}(s)}$ onde assumimos que a função de distribuição de $T$, denotada por $F(s)$ é absolutamente contínua com densidade $f(s)$ e função de sobrevivência $\bar{F}(s)=1-F(s)$.

Em nossa abordagem consideramos a informação mais geral

$$
\Im_{t}=\sigma\left\{I\left(S_{i}>s\right), s \leq t, i=1, \ldots, n\right\}
$$

onde $n$ é o número de componentes do sistema, isto é, observamos se o i-ésimo componente sobrevive ou não ao instante $t,\left\{S_{i}>t\right\}$, e conhecemos o instante da falha se esta ocorre antes de $t$. Tal filtragem é conhecida como a pré-t-história dos componentes.

Em relação a qualquer familia de $\sigma$-álgebras, Jacob (1975) prova a existência de uma correspondência biunívoca entre a distribuição conjunta dos tempos de vidas dos componentes $\left(S_{1}, \ldots, S_{n}\right)$ e de seus compensadores nos pontos finais

$$
\left(A_{1}\left(S_{1}\right), \ldots, A_{n}\left(S_{n}\right)\right), A_{i}(t)=\int_{0}^{t \wedge S_{i}} \lambda_{s}(i) d s
$$

que são variáveis aleatórias independentes e identicamente distribuidas com distribuição exponencial de parâmetro 1 , mesmo que exista qualquer relação de dependência entre os componentes, desde que não admitamos falhas simultâneas a menos de um conjunto de medida nula. 
Em certos casos vale a fórmula exponencial, isto é, a função de sobrevivência de um certo tempo de vida $T$ pode ser escrita da seguinte forma

$$
\bar{F}(t)=\prod_{s \leq t}(1-\triangle A(s)) e^{-A^{c}(t)}
$$

onde $\triangle A(s)=A(s)-A\left(s^{-}\right)$e $A^{c}(t)=A(t)-\sum_{s \leq t} \triangle A(s)$ são os saltos e a parte contínua do compensador $A(t)$, respectivamente e neste caso a distribuição do tempo de vida $T$ é determinada completamente pelo seu compensador.

Quando $\Im_{t}=\sigma\left\{I\left(S_{i}>s\right), s \leq t, i=1, \ldots, n\right\}$, Arjas (1981), mostra uma relação entre o compensador do sistema $A(t)$ e o compensador dos componentes $A_{i}(t), i=$ $1, \ldots, n$, que é a seguinte:

$$
A(t)=\sum_{i=1}^{n}\left(A_{i}(t \wedge T)-A_{i}\left(Y_{i}\right)\right)^{+}, q . c .,
$$

onde, $a^{+}=\max \{0, a\}$ e $Y_{i}$ é o nível crítico do componente $i$, isto é, o primeiro instante a partir do qual o sistema falha se o componente $i$ falha.

Segue dos parágrafos acima que podemos estudar o tempo de vida do sistema através de seu compensador e analisar este compensador através dos compensadores dos componentes.

Nesta tese abordaremos três temas principais que generalizam alguns resultados clássicos da teoria da confiabilidade, todos associados a um modelo de tempo de vida geral. Na primeira parte abordaremos o conceito de importância de Barlow e Proschan da confiabilidade de um componente para a confiabilidade do sistema. Em uma segunda parte, considerando as dinâmicas de informações e observando o sistema a níveis de seus componentes, definimos uma medida de importância através dos processos de ganho de inovações, generalizando um resultado de Norros (1986b). Finalizaremos este trabalho analisando modelos de Burn-in envolvendo processos de 
custos e ganhos, onde encontramos um tempo ótimo de parada, baseado em uma regra de parada chamada de regra de parada IOPF ( infinitesimal olhada para frente).

No primeiro capítulo descrevemos brevemente as medidas de importâncias de Birnbaum (1969), Barlow e Proschan (1975) e a de Iyer (1992). Propomos uma nova definição de medida de importância através de compensadores, denominada por "Medidas de Importâncias para Blocos". Mostramos que tal medida generaliza a medida proposta por Iyer (1992) que por sua vez generaliza a medida de Barlow e Proschan, pois não necessita da hipótese de que os tempos de vidas dos componentes de um sistema sejam independentes, hipótese esta essencial na definição de Barlow e Proschan e também relaxar a condição que tenham funções de distribuição absolutamente contínuas que é uma das condições da medida proposta por Iyer. Mostramos ainda que esta nova medida preserva algumas propriedades (mesmo no caso de dependência) da medida de Barlow e Proschan, tais como as propriedades obtidas pelas Proposições (1.17) e (1.18) e a do Teorema (1.28). Nossa contribuição neste capítulo são os resultados alcançados a partir da Seção 1.3.2.

No segundo capítulo consideramos as falhas dos componentes de um sistema como um processo de contagem multivariado. Observando as suas pré-t-histórias e usando o processo de ganho de inovação da Teoria da Inovação (veja Brémaud (1982)) definimos uma medida de importância que, para um caso particular, é a medida proposta por Norros (1986b). Além disso, pela fórmula de recorrência descrevemos o processo ganho de inovação para cadeias de Markov não homogêneas (através do Teorema 2.3), o que generaliza um resultado de Brémaud (1982). No caso em que a cadeia de Markov tem somente dois estados obtemos um valor explicito para o ganho de inovação. A nossa principal contribuição neste capítulo são as 
provas dos Teoremas (2.3) e (2.6).

No terceiro capítulo detalhamos um modelo de burn-in proposto por Aven e Jensen (1999), baseado nas informações dos tempos de vida de $m$ objetos colocados em testes. Usaremos uma representação semimartingal regular (SMR), ou seja, um modelo de tempo de vida geral do tipo (1), para determinar os tempos ótimos de burn-in. Consideramos uma estrutura de custo, devido às falhas ao longo do tempo, descritas por um processo $\left(Z_{t}\right)_{t \geq 0}$ e uma estrutura de ganho por unidade de tempo, caso não haja falhas, determinada por um processo $\left(X_{t}\right)_{t \geq 0}$. Analisamos o problema de burn-in segundo dois critérios:

Problema 1: Encontrar o tempo de burn-in ( $\Im_{t}$-tempo de parada) ótimo que maximize a diferença entre os processos de ganho e de custo, isto é, $K_{t}^{1}=X_{t}-Z_{t}$.

Problema 2: Encontrar o tempo de burn-in ( $\Im_{t}$-tempo de parada) ótimo que minimize a razão entre os processos de custo e de ganho, isto é, $K_{t}^{2}=\frac{Z_{t}}{X_{t}}$.

A nossa contribuição neste capítulo é a proposta e a solução para o Problema 2.

Finalmente, no Apêndice apresentaremos algumas definições e alguns resultados relacionados a teoria de processos estocásticos e a teoria clássica de confiabilidade. 


\section{Capítulo 1}

\section{Medidas de Importância}

\subsection{Introdução}

Existem duas razões principais que nos levam a determinar uma medida de importância de um componente para o sistema. Primeiramente, nos permite que possamos determinar qual é "mérito"do componente na análise de otimização da confiabilidade do sistema a um custo ou esforço mínimo. Além disso, permite gerar uma lista dos componentes mais propensos a causar a falha do sistema, auxiliando assim uma política de manutenção eventual.

Birnbaum (1969) define a importância da confiabilidade de um componente para a confiabilidade do sistema da seguinte maneira: Sejam $T$ e $S_{i}$ os tempos de vidas do sistema e do componente $i$ respectivamente. Então a importância de $S_{i}$ para $T$ no instante $t$ é definida por:

$$
I^{B}(i, t)=P\left(T>t \mid S_{i}>t\right)-P\left(T>t \mid S_{i} \leq t\right) .
$$

Observe que esta medida depende de um determinado instante $t$, no tempo e portanto contém limitações nas aplicações. Para resolver tal problema várias medidas de importância foram sugeridas (Barlow e Proschan (1975), Natvig (1985), e ou- 
tros), sendo que a maioria delas são integrais ponderadas de $I^{B}(i, t)$ sobre $t$. Sob a hipótese de independência, Barlow e Proschan (1975) define a importância da confiabilidade do componente $i$ para a confiabilidade do sistema como $\int_{0}^{\infty} I^{B}(i, t) d F_{i}(t)$ onde $F_{i}(t)$ é a função de distribuição absolutamente contínua do tempo de vida $S_{i}$, $i=1, \ldots, n$.

Existe uma relação bem conhecida entre a classe de todas as funções de distribuições e a classe dos $\Im_{t}$-compensadores, onde $\Im_{t}$ é a $\sigma$-álgebra natural dos componentes (Norros (1986a)), ou seja, $\Im_{t}$ é a pré-t-história dos componentes. Usaremos esta relação para considerar a dependência entre os componentes e a dependência estrutural na formulação de uma medida que generaliza a Importância de Barlow e Proschan, através dos compensadores.

\subsection{Medida de Importância para Componentes Independentes}

Interpretaremos o instante inicial 0 (zero) como sendo o tempo em que o sistema $\Phi$ começa a funcionar. Se o vetor dos tempos de vida dos componentes é denotado por $\mathbf{S}=\left(S_{1}, \ldots, S_{n}\right)$, o tempo de vida do sistema pode ser descrito como sua decomposição série-paralelo como em (A.23),

$$
T=\Phi(\mathbf{S})=\min _{1 \leq j \leq k} \max _{i \in K_{j}} S_{i}
$$

onde $K_{j}, j=1, \ldots, k$ são os conjuntos de cortes mínimais (veja $\left.(A .30)\right)$ e $\Phi$ é chamada função de estrutura do sistema. Podemos considerar também tal decomposição, usando a seguinte notação equivalente:

$$
X(t)=\Phi\left(\mathbf{X}_{t}\right)=\min _{1 \leq j \leq k} \max _{i \in K_{j}} X_{t}(i)
$$


onde $(X(t))_{t \geq 0}$ e $\left(X_{t}(i)\right)_{t \geq 0}$ são processos estocásticos contínuos à direita, com $X(t)=I(T>t), X_{t}(i)=I\left(S_{i}>t\right)$ e $\mathbf{X}_{t}=\left(X_{t}(1), X_{t}(2), \ldots, X_{t}(n)\right)$.

Um componente $i$ é crítico para o sistema no tempo $t$ se

$$
\Phi\left(1_{i}, \mathbf{X}_{t}\right)-\Phi\left(0_{i}, \mathbf{X}_{t}\right)=1
$$

onde $\left({ }_{i}, \mathrm{X}_{t}\right)=\left(X_{t}(1), \ldots, X_{t}(i-1),{ }_{. i}, X_{t}(i+1), \ldots, X_{t}(n)\right)$.

Definição 1.1. (Birnbaum (1969))

A importância da confiabilidade do componente $i$ para a confiabilidade do sistema no tempo t é a probabilidade do componente ser crítico no tempo $t$, i.e.:

$$
\begin{aligned}
I^{B}(i, t) & =P\left(\Phi\left(1_{i}, \mathbf{X}_{t}\right)-\Phi\left(0_{i}, \mathbf{X}_{t}\right)=1\right) \\
& =P\left(T>t \mid S_{i}>t\right)-P\left(T>t \mid S_{i} \leq t\right) .
\end{aligned}
$$

Se os componentes forem independentes, a confiabilidade do sistema é uma função multilinear das funções de distribuição dos tempos de vidas dos componentes, em outras palavras, $\bar{F}(t)=h(\overline{\mathbf{F}}(t))$, onde $\overline{\mathbf{F}}(t)=\left(\bar{F}_{1}(t), \ldots, \bar{F}_{n}(t)\right), F_{1}, F_{2}, \ldots, F_{n}$ são as funções de distribuições de $S_{1}, \ldots, S_{n}$ repectivamente, $F$ é a função de distribuição do tempo de vida do sistema e $h: \mathbb{R}_{+}^{n} \rightarrow \mathbb{R}_{+}$é uma função multilinear. Para um $t$ fixo, é fácil provar que

$$
I^{B}(i, t)=\frac{d h(\overline{\mathbf{F}}(t))}{d \bar{F}_{i}(t)},
$$

isto é, $I^{B}(i, t)$ pode ser interpretada como a taxa de crescimento da confiabilidade do sistema em razão do crescimento da confiabilidade do componente.

Note que, se os componentes forem independentes, temos

$$
\frac{d F(t)}{d t}=-\frac{d h(\overline{\mathbf{F}}(t))}{d t}=-\sum_{i=1}^{n} \frac{d h(\overline{\mathbf{F}}(t))}{d \bar{F}_{i}(t)} \frac{d \bar{F}_{i}(t)}{d t}=\sum_{i=1}^{n} I^{B}(i, t) \frac{d F_{i}(t)}{d t}
$$


portanto

$$
E[I(T \leq t)]=P(T \leq t)=\sum_{i=1}^{n} \int_{0}^{t} I^{B}(i, s) d F_{i}(s),
$$

i.e., podemos melhorar a confiabilidade do sistema através de $\int_{0}^{t} I^{B}(i, s) d F_{i}(s), i=$ $1, \ldots, n$.

A importância de confiabilidade de Birnbaum para um componente é uma função mensurável que depende de um determinado tempo $t$. Barlow e Proschan (1975) define uma medida de importância que independe do tempo.

Definição 1.2. (Barlow e Proschan (1975))

Seja $T=\Phi\left(S_{1}, \ldots, S_{n}\right)$ o tempo de vida de um sistema coerente de $n$ componentes com tempos de vida independentes, $S_{1}, \ldots, S_{n}$, com funções de distribuições absolutamente contínuas, $F_{1}, \ldots, F_{n}$, respectivamente. A importância da confiabilidade de Barlow e Proschan do componente i para a confiabilidade de sistema é definida por,

$$
I^{B P}(i)=\int_{0}^{\infty} I^{B}(i, t) d F_{i}(t)
$$

onde $I^{B}(i, t)$ é a importância de confiabilidade do componente de Birnbaum.

Iyer (1992) generaliza a definição de Barlow e Proschan, considerando o caso onde $S_{1}, \ldots, S_{n}$ são dependentes e com distribuições absolutamente contínuas, mas $P\left(S_{i}=S_{j}\right)=0$ se $i \neq j$, i.e., não admite falhas multiplas. 
Definição 1.3. (Iyer (1992))

Seja $T=\Phi\left(S_{1}, \ldots, S_{n}\right)$ o tempo de vida do sistema, onde $S_{i}, i=1, \ldots, n$ tem distribuição conjunta absolutamente contínua. A importância da confiabilidade do componente i para confiabilidade do sistema é

$$
I(i)=\int_{0}^{\infty} P\left(\Phi\left(1_{i}, \mathbf{X}_{t}\right)-\Phi\left(0_{i}, \mathbf{X}_{t}\right)=1 \mid S_{i}=t\right) d F_{i}(t)
$$

Observação 1.4. Observe que esta medida correponde à de Barlow e Proschan quando os componentes são independentes e absolutamente contínuos.

\subsection{Medida de Importância para Blocos}

\subsubsection{Processos Pontuais Marcados}

Antes de definirmos uma medida que generaliza a medida definida por Iyer (1992)(veja Definição 1.3) descrita na subseção anterior, vamos descrever alguns resultados de processos pontuais marcados.

Suponha que o sistema seja monitorado ao nível dos componentes, $C_{1}, \ldots, C_{n}$, ou seja, a cada momento $t$ o observador sabe se o evento $\left\{S_{i} \leq t\right\}$ (o componente $C_{i}$ falhou em um instante menor ou igual a $t$ ), $1 \leq i \leq n$, ocorre ou não e se ele ocorre, sabe-se exatamente o valor de $S_{i}$. A formulação matemática deste conhecimento é determinada através de uma família de sub- $\sigma$-álgebras de $\Im$, denotada por $\left(\Im_{t}\right)_{t \geq 0}$ onde

$$
\Im_{t}=\sigma\left\{I\left(S_{i}>s\right): 1 \leq i \leq n, s \leq t\right\}
$$

satisfazendo as condições usuais de Dellacherie, isto é, são contínuas à direita e completas (contêm todos os eventos de medida nula). Vamos descrever as falhas dos componentes $C_{1}, \ldots, C_{n}$ que compoêm o sistema que aparecem no decorrer do 
tempo, através de um processo pontual marcado multivariado (veja Definição $(A .4)$ ). Assim, a relação do tempo de vida de um sistema $T$ com os tempos de vida de seus componentes pode ser pensada através de um processo pontual simples derivado de um processo pontual multivariado.

Para qualquer ocorrência $S_{1}(w), \ldots, S_{n}(w)$, seja $q(\omega)$ o número de valores distintos no conjunto $\left\{S_{i}(w) ; 1 \leq i \leq n\right\}$. Denotaremos as estatística de ordem crescente desse conjunto por

$$
T_{(1)}<T_{(2)}<\ldots<T_{(q(w))}
$$

Seja também

$$
J_{(k)}(w)=\left\{i: S_{i}(w)=T_{(k)}(w), 1 \leq i \leq n\right\}
$$

o conjunto de componentes que falham no k-ésimo tempo de falha $T_{(k)}$. Se não existirem falhas múltiplas, os valores de $J_{(k)}$ é um dos conjuntos $\{i\}, i=1, \ldots, n$, mas em geral, $J_{(k)}$ é um conjunto aleatório, com valores em $\Lambda$, onde $\Lambda$ é o conjunto das partes diferente do vazio de $\{1,2, \ldots, n\}$. Chamaremos $T_{(k)}$ o k-ésimo tempo de falha e $J_{(k)}$ o conjunto de componentes que falham em $T_{(k)}$, o qual denominaremos "blocos". Assim a sequência $\left(T_{(k)}, J_{(k)}\right)_{1 \leq k \leq q}$ descreve como os componentes $C_{1}, \ldots, C_{n}$, falharam. Considerando

$$
\begin{gathered}
T_{(q+1)}=T_{(q+2)}=\ldots=\infty \\
J_{(q+1)}=J_{(q+2)}=\ldots=\emptyset,
\end{gathered}
$$

temos:

Definição 1.5. Definimos o Processo de Falhas dos Componentes como o processo pontual multivariado $\left(T_{(k)}, J_{(k)}\right)_{k \geq 1}$. 
Uma maneira equivalente de descrever esse processo é através dos processos de contagem. Para cada conjunto de falha $\emptyset \neq J \in \Lambda$ fixado, sejam $T_{J}$ e $N_{J}(w ; t)$ definidos por:

$$
T_{J}=\inf \left\{T_{(k)}: J_{(k)}=J\right\}
$$

onde $\inf \emptyset=\infty \mathrm{e}$

$$
N_{J}(w, t)= \begin{cases}0 & \text { if } t<T_{J}(w) \\ 1 & \text { if } t \geq T_{J}(w)\end{cases}
$$

Cada realização $\left(S_{i}(w)\right)_{1 \leq i \leq n}$ determina uma realização do processo

$$
\left(N_{J}(t) ; J \in \Lambda\right)_{t \geq 0}
$$

e vice-versa, isto é

$$
\Im_{t}=\sigma\left\{N_{J}(s) ; s \leq t, J \in \Lambda\right\}
$$

é equivalente a

$$
\Im_{t}=\sigma\left\{I\left(S_{i}>s\right), 1 \leq i \leq n, s \leq t\right\}
$$

Na Teoria da Confiabilidade, além de analisarmos o processo das falhas dos componentes, estamos interessados na consequência dessas falhas no tempo de vida do sistema. Podemos descrever o tempo de falha do sistema, que depende das falhas acumuladas dos componentes, da seguinte maneira. Se $\Phi$ é um sistema monótono (ou coerente) com tempo de vida $T$, defina

$$
D(t)= \begin{cases}J_{(1)} \cup \ldots \cup J_{(k)}, & \text { se } T_{(k)} \leq t<T_{(k+1)} \\ \emptyset & \text { se } t<T_{(1)}\end{cases}
$$

o conjunto acumulado de blocos (falhas de componentes) até o tempo $t$. É fácil notar que, para cada $\omega$ fixado $D(w, t)$ é contínua à direita e crescente na ordem parcial de $\Lambda$. Podemos sempre definir $D(t-)=\lim _{s \uparrow t} D(s)$. 
Se $\Lambda_{\Phi}=\left\{K_{1}, \ldots, K_{k}\right\}$ é a coleção de todos os conjuntos de cortes de $\Phi$ (veja Definição A.30), o tempo de vida do sistema $T$, pode ser obtido da seguinte forma:

$$
T=\inf \left\{t \geq 0: D(t) \in \Lambda_{\Phi}\right\}=\min \left\{T_{(k)}: J_{(1)} \cup \ldots \cup J_{(k)} \in \Lambda_{\Phi}\right\}
$$

Portanto podemos pensar no processo pontual da falha do sistema, definido por

$$
N_{\Phi}(t)=I(T \leq t), t \geq 0
$$

como um processo de contagem derivado do processo pontual multivariado

$$
\left(T_{(k)}, J_{(k)}\right)_{k \geq 1}
$$

De outro ponto de vista, podemos fixar um tempo $t$ e observarmos quais os blocos que falharam em $d t$ e que resultaria na falha do sistema. Vamos denotar a classe

$$
\Gamma_{\Phi}(t)=\left\{J \in \Lambda_{\Phi}: D(t-) \notin \Lambda_{\Phi}, D(t-) \cup J \in \Lambda_{\Phi}\right\}
$$

dos blocos que são críticos para o sistemas, no instante $t$. Temos que para cada $\omega$, a função $t \rightarrow \Gamma_{\Phi}(t)$ é crescente na ordem parcial de $\Lambda$ para $t \leq T$, e contínua à esquerda. Observemos que o sistema falha em $t$ quando ocorre algum conjunto crítico em $\Gamma_{\Phi}(t)$ e vice-versa, isto é

$$
\{T \in(t, t+d t]\}=\bigcup_{J \in \Gamma_{\Phi}(t)}\left\{T_{J} \in(t, t+d t]\right\}
$$

onde os eventos $\left\{T_{J} \in(t, t+d t]\right\}$ são disjuntos.

Por conveniência, vamos definir o nível crítico de falha do bloco $J$, como o primeiro instante no qual a falha de $J$ causa a falha do sistema, isto é, o $\Im_{t}$ - tempo de parada $Y_{\Phi}(J)$ definido por

$$
\begin{aligned}
Y_{\Phi}(J) & =\inf \left\{t \geq 0: J \in \Gamma_{\Phi}(t)\right\} \\
& =\inf \left\{t \geq 0: D(t) \cup J \in \Lambda_{\Phi}\right\}
\end{aligned}
$$


onde, por convenção, $\inf \emptyset=\infty$.

Claramente temos

$$
\left\{J \in \Gamma_{\Phi}(t)\right\}=\left\{Y_{\Phi}(J)<t \leq T\right\}
$$

Na Teoria dos Martingais é natural colocar a noção de risco em termos da intensidade estocástica, ou na sua forma integral em termos dos compensadores ( veja Definição A.5) dos processos de contagem $\left(N_{J}(t) ; J \in \Lambda\right)_{t \geq 0}\left(\mathrm{e} / \mathrm{ou}\left(N_{\Phi}(t)\right)_{t \geq 0}\right)$. Para $J \in \Lambda$, o $\Im_{t}$-compensador $\left(A_{J}(t)\right)_{t \geq 0}$ do processo de contagem univariado $\left(N_{J}(t)\right)_{t \geq 0}$ é único, $P$-q.c., contínuo à direita, crescente, previsível, e tal que, para cada $k \geq 1$, o processo diferença até o tempo $T_{(k)}$,

$$
\left.N_{J}\left(t \wedge T_{(k)}\right)-A_{J}\left(t \wedge T_{(k)}\right)\right)
$$

é um $\Im_{t}$-martingal, uniformemente integrável e de média zero. Como $T_{(q+1)}=\infty$, temos que

$$
M_{J}(t)=N_{J}(t)-A_{J}(t)
$$

é um $\Im_{t}$-martingal, uniformemente integrável e de média zero, o que nos permite concluir que

$$
E A_{J}(t)=P\left(T_{J} \leq t\right)
$$

Um interpretação bem conhecida para tal compensador ( veja Aven e Jensen (1999)) é que

$$
A_{J}(d t)=P\left(T_{J} \in(t, t+d t] \mid \Im_{t^{-}}\right) .
$$

Intuitivamente, isto corresponde a predizer se $T_{J}$ vai acontecer "agora," baseado em todas as observações disponíveis até o presente, mas não incluindo o mesmo. Motivado por isto, chamamos $\left(A_{J}(t)\right)_{t \geq 0}$ o processo de risco do bloco $J$ e $\left(A_{J}(t) ; J \in\right.$ $\Lambda)_{t \geq 0}$ o processo de risco multivariado. 
Da mesma forma, podemos estudar o $\Im_{t}$-compensador do processo de contagem $\left(N_{\Phi}(t)\right)_{t \geq 0}$ da falha do sistema, denotado por $\left(A_{\Phi}(t)\right)_{t \geq 0}$. Por razões óbvias, chamaremos este compensador como o processo de risco do sistema. É natural perguntar qual é a contribuição da falha de um bloco para predizer a falha do sistema. Respondendo a esta pergunta, Arjas (1981) caracteriza a relação entre os $\Im_{t^{-}}$compensador dos blocos e o $\Im_{t}$-compensador do sistema, conforme o teorema abaixo.

Teorema 1.6. (Arjas (1981))

Seja $\Phi$ um sistema coerente com tempo de vida $T$ e $\Im_{t}$ é a pré-t-história dos seus componentes então o $\Im_{t}$-compensador do processo $N_{\Phi}(t)=I(T \leq t)$ é

$$
A_{\Phi}(t)=\sum_{J \in \Lambda}\left[A_{J}(t \wedge T)-A_{J}\left(Y_{\Phi}(J)\right)\right]^{+}, \text {q.c. }
$$

onde $[a]^{+}=\max \{a, 0\}$ e $A_{J}(t)$ é os $\Im_{t}$-compensador do processo

$$
N_{J}(t)=I\left(T_{J} \leq t\right), J \in \Lambda \text {. }
$$

Meyer (1971) mostra que sob algumas condições os compensadores dos tempos de vidas dos componentes de um sistema monótono, nos seus tempos finais são variáveis aleatórias i.i.d. com distribuição exponenciais de parâmetro 1, ou seja:

Proposição 1.7. (Meyer (1971))

Sejam $S_{i}, i=1, \ldots, n$ os tempos de vida dos componentes que compõem um sistema coerente $\Phi$ e $\Im_{t}$ a pré-t-história destes componentes. Suponha que as seguintes condições estão satisfeitas:

(i) $P\left(0<S_{i}<\infty\right)=1, i=1, \ldots, n$,

(ii) $P\left(S_{i}=S_{j}\right)=0, i \neq j, i=1, \ldots, n, j=1, \ldots, n$, 
(iii) Os $\Im_{t}$-compensadores $A_{\{i\}}(t)=A_{i}(t)$ correspondentes aos processo

$$
N_{i}(t)=I\left(S_{i} \leq t\right), t \geq 0, i=1, \ldots, n
$$

são contínuos.

Então os $\Im_{t}$-compensadores nos tempos finais, $A_{i}(\infty)=A_{i}\left(S_{i}\right), i=1, \ldots, n$, são variáveis aleatórias independentes e exponencialmente distribuídas com parâmetro 1.

\subsubsection{Medida de Importância através dos Compensadores}

Generalizando a notação do capítulo anterior, seja $\mathbf{x}=\left(x_{1}, x_{2}, \ldots, x_{n}\right) \in\{0,1\}^{n}$ e $J=\left\{j_{1}, j_{2}, \ldots, j_{r}\right\} \subset\{1,2, \ldots, n\}$. Denotamos os vetores $\mathrm{n}$-dimensionais $\left(\mathbf{1}_{J}, \mathbf{x}\right) \mathrm{e}$ $\left(\mathbf{0}_{J}, \mathbf{x}\right)$ pertencente a $\{0,1\}^{n}$ como os conjuntos onde os componentes correspondentes aos índices $j_{1}, j_{2}, \ldots, j_{r}$ de $\mathbf{x}$ são formados por 1's e 0's respectivamente. Note que dessa forma, um bloco $J$ é crítico para o sistema no tempo $t$, se

$$
\Phi\left(\mathbf{1}_{\mathbf{J}}, \mathbf{X}_{t}\right)-\Phi\left(\mathbf{0}_{\mathbf{J}}, \mathbf{X}_{t}\right)=1
$$

Um forma equivalente para a equação (1.10) obtida diretamente pela equações (1.7) e (1.8) é dado por,

$$
\left\{Y_{\Phi}(J)<t \leq T\right\}=\left\{\Phi\left(\mathbf{1}_{\mathbf{J}}, \mathbf{X}_{t}\right)-\Phi\left(\mathbf{0}_{\mathbf{J}}, \mathbf{X}_{t}\right)=1\right\}
$$

Sob o conjunto $\left\{T_{J}=t\right\}$ podemos definir:

Definição 1.8. A importância da confiabilidade do bloco $J$ (no conjunto $\left\{T_{J}=t\right\}$ ) para a confiabilidade do sistema no tempo t é a probabilidade que $J$ seja crítico para o sistema em t, isto é

$$
I_{C}^{B}(J, t)=P\left(Y_{\Phi}(J)<t \leq T \mid T_{J}=t\right)
$$


Observação 1.9. No caso onde não existem falhas simultâneas, $J_{(k)}=\{i\}$, para algum $i \in\{1,2, \ldots, n\}$, e portanto $T_{\{i\}}=S_{i}$,

$$
I_{C}^{B}(\{i\}, t)=P\left(\Phi\left(1_{i}, \mathbf{X}_{t}\right)-\Phi\left(0_{i}, \mathbf{X}_{t}\right)=1 \mid S_{i}=t\right),
$$

como aparece na definição 1.3. Se os componentes são independentes, $I_{C}^{B}(\{i\}, t)$ é a importância de Birnbaum (1.1).

Aplicando a noção de criticalidade (confira (1.10)), podemos generalizar a medida de importância de Barlow e Proschan.

Definição 1.10. Seja $T$ o tempo de vida de um sistema coerente $\Phi$, e seja $T_{J}$ o tempo de vida do bloco $J$ com $\Im_{t}$-compensador $A_{J}(t)$. A importância da confiabilidade do bloco $J$ para a confiabilidade do sistema é definida por

$$
I^{\Phi}(J)=E\left[\left[A_{J}(T)-A_{J}\left(Y_{\Phi}(J)\right)\right]^{+}\right]
$$

onde $Y_{\Phi}(J)$ é o nível crítico do bloco $J$.

No caso onde $T_{J}, J \in \Lambda$ é um $\Im_{t}$-tempo de parada totalmente inacessível (veja Definição $A .14)$, o Lema $A .15$ nos garante que o $\Im_{t}$-compensador é contínuo e

$$
\begin{aligned}
E\left[A_{J}(t \wedge T)-A_{J}\left(Y_{\Phi}(J)\right)\right]^{+} & =E\left[\int_{Y_{\Phi}(J)}^{T \wedge t} d A_{J}(s)\right]^{+} \\
& =E\left[\int_{0}^{t} I\left(Y_{\Phi}(J)<s \leq T\right) d A_{J}(s)\right] .
\end{aligned}
$$

Se os componentes são independentes, então não existe falhas multiplas e os $J_{(k)}$ são conjuntos unitarios $\{i\} \in\{1, \ldots, n\}$. Assim o $\Im_{t}$-compensador do tempo de vida $S_{i}$ é $A_{i}(t)=-\log \left(\overline{F_{i}}\left(t \wedge S_{i}\right)\right)$, onde denotamos por $\overline{F_{i}}(t)=1-F_{i}(t)$ a função de 
sobrevivência do componente $i$ é a confiabilidade do componente $i$ para um tempo fixo $t$. Temos a seguinte proposição:

Proposição 1.11. Se os componentes são independentes com distribuição absolutamente contínuas, a importância da confiabilidade do componente $i$ para a confiabilidade do sistema é

$$
I^{\Phi}(\{i\})=I^{B P}(i)=\int_{0}^{\infty} I^{B}(i, t) d F_{i}(t) .
$$

\section{Prova:}

Por hipótese temos, $I_{C}^{B}(\{i\}, t)=I^{B}(i, t)$ e $Y_{\Phi}(\{i\})=Y_{\Phi}(i)$. Logo,

$$
\begin{aligned}
I^{\Phi}(\{i\}) & =E\left[\int_{0}^{\infty} I\left(Y_{\Phi}(\{i\})<t \leq T\right) d\left(-\log \bar{F}_{i}\left(t \wedge S_{i}\right)\right)\right] \\
& =E\left[E\left[\int_{0}^{S_{i}} I\left(Y_{\Phi}(\{i\})<t \leq T\right) d\left(-\log \left(\bar{F}_{i}(t)\right)\right)\right]\right] \\
& =E\left[\int_{0}^{\infty}\left[\int_{0}^{s} I\left(Y_{\Phi}(\{i\})<t \leq T\right) \frac{d F_{i}(t)}{\bar{F}_{i}(t)}\right] d F_{i}(s)\right] \\
& =E\left[\int_{0}^{\infty}\left[\int_{t}^{\infty} d F_{i}(s)\right] I\left(Y_{\Phi}(\{i\})<t \leq T\right) \frac{d F_{i}(t)}{\bar{F}_{i}(t)}\right] \\
& =E\left[\int_{0}^{\infty} I\left(Y_{\Phi}(i)<t \leq T\right) d F_{i}(t)\right] \\
& =\int_{0}^{\infty} P\left(\Phi\left(1_{i}, \mathbf{X}_{t}\right)-\Phi\left(0_{i}, \mathbf{X}_{t}\right)=1 \mid S_{i}=s\right) d F_{i}(t) \\
& =\int_{0}^{\infty} I^{B}(i, t) d F_{i}(t) .
\end{aligned}
$$

Observe que independência não é uma condição necessária para ambas medidas, como podemos ver na proposição abaixo. 
Proposição 1.12. Sob a notação acima, se $T_{J}$ é um tempo de parada totalmente inacessivel, temos $I^{\Phi}(J)=\int_{0}^{\infty} I_{C}^{B}(J, t) d F_{J}(t)$.

\section{Prova:}

Primeiramente observe que

$$
\int_{0}^{t} I\left(Y_{\Phi}(J)<s \leq T\right) d\left[N_{J}(s)-A_{J}(s)\right]
$$

é um $\Im_{t}$-martingal, pois $I\left(Y_{\Phi}(J)<s \leq T\right)$ é contínuo à esquerda e portanto $\Im_{t^{-}}$ previsivel. Logo

$$
\begin{aligned}
I^{\Phi}(J) & =E\left[\int_{0}^{\infty} I\left(Y_{\Phi}(J)<s \leq T\right) d A_{J}(s)\right] \\
& =E\left[\int_{0}^{\infty} I\left(Y_{\Phi}(J)<s \leq T\right) d N_{J}(s)\right]=P\left(Y_{\Phi}(J)<T_{J} \leq T\right) \\
& =\int_{0}^{\infty} P\left(\Phi\left(1_{J}, \mathbf{X}_{s}\right)-\Phi\left(0_{J}, \mathbf{X}_{s}\right)=1 \mid T_{J}=s\right) d F_{J}(s) \\
& =\int_{0}^{\infty} I_{C}^{B}(J, s) d F_{J}(s) .
\end{aligned}
$$

Observação 1.13. Segue-se dos resultados acima, que a medida $I^{\Phi}(J), J \in \Lambda$ é uma generalização da medida de Barlow e Prochan, onde sob hipótese de independência temos $I^{\Phi}(\{i\})=I^{B P}(i)=I(i)$ e sob hipótese de não existir falhas multiplas temos $I^{\Phi}(\{i\})=I(i)$.

A seguir veremos exemplos que ilustram os resultados obtidos:

Exemplo 1.14. Vamos analisar um sistema de dois componentes dependentes $C_{1}, C_{2}$. Considere a função de distribuição bivariada de Marshall e Olkin, com parâmetros 
$\beta_{1}>0, \beta_{2}>0$ e $\beta_{12} \geq 0$, dada por

$$
\begin{aligned}
P\left(S_{1} \leq s, S_{2} \leq t\right) & =1-e^{-\left(\beta_{1}+\beta_{12}\right) s}-e^{-\left(\beta_{2}+\beta_{12}\right) t} \\
& +e^{-\left(\beta_{1} s+\beta_{2} t+\beta_{12}(s \vee t)\right)}
\end{aligned}
$$

onde $S_{1}$ e $S_{2}$ são os tempos de vidas dos componentes $C_{1}, C_{2}$ respectivamente. Uma interpretação para esta distribuição é a seguinte. Três variáveis $Z_{1}, Z_{2}, Z_{12}$, independentes com distribuição exponenciais de parâmetros $\beta_{1}>0, \beta_{2}>0$ e $\beta_{12} \geq 0$, respectivamente, descrevem tempos de choques que causam as falhas dos componentes $C_{1}$ ou $C_{2}$, com tempos de vidas $S_{1}=Z_{1} \wedge Z_{12}$ e $S_{2}=Z_{2} \wedge Z_{12}$.

Pode-se distinguir três blocos distíntos, $\{1\},\{2\},\{12\}$. Note por exemplo, que $T_{\{1\}} \neq S_{1}$ q.c., pois $T_{\{1\}}=\infty$ em $\left\{S_{1}=S_{2}\right\}$ isto é, em $\left\{Z_{12} \leq Z_{1} \vee Z_{2}\right\}$. Calculando os seus compensadores

$$
A_{\{1\}}(t)=\left\{\begin{array}{lc}
\beta_{1} t & \text { em }\left\{S_{1}>t, S_{2}>t\right\} \\
\left(\beta_{1}+\beta_{12}\right) t & \text { em }\left\{S_{1}>t, S_{2} \leq t\right\} \\
0 & \text { caso contrario }
\end{array}\right.
$$

$A_{\{2\}}(t)$ é obvio, por simetria, $e$

$$
A_{\{12\}}(t)= \begin{cases}\beta_{12} t & \text { em }\left\{S_{1}>t, S_{2}>t\right\} \\ 0 & \text { caso contrario }\end{cases}
$$

As distribuições marginais são exponenciais, onde $P\left(S_{i} \leq t\right)=1-e^{-\left(\beta_{i}+\beta_{12}\right) s}, i=$ 1,2. Se o sistema é série, seu tempo de vida é $T=S_{1} \wedge S_{2}$ com função de distribuição $P(T \leq t)=1-e^{-\left(\beta_{1}+\beta_{2}+\beta_{12}\right) t}$.

O nível crítico do bloco $\{1\}$ é $Y_{\Phi}(\{1\})=0$, e seu compensador é

$$
A_{\{1\}}(t)=\beta_{1} t I\left(S_{1}>t, S_{2}>t\right)+\left(\beta_{1}+\beta_{12}\right) t I\left(S_{1}>t, S_{2} \leq t\right)
$$


portanto

$$
\begin{aligned}
I^{\Phi}(\{1\}) & =E\left[\int_{0}^{\infty} I\left(0<s \leq S_{1} \wedge S_{2}\right) d A_{\{1\}}(s)\right] \\
& =E\left[\int _ { 0 } ^ { \infty } I ( 0 < s \leq S _ { 1 } \wedge S _ { 2 } ) \left[\beta_{1} I\left(S_{1}>s, S_{2}>s\right)\right.\right. \\
& \left.\left.+\left(\beta_{1}+\beta_{12}\right) I\left(S_{1}>s, S_{2} \leq s\right)\right] d s\right] \\
& =\beta_{1} E\left[S_{1} \wedge S_{2}\right] \\
& =\frac{\beta_{1}}{\beta_{1}+\beta_{2}+\beta_{12}} .
\end{aligned}
$$

O nível crítico do bloco $\{12\}$ é também 0 , e $A_{\{12\}}(t)=\beta_{12} t I\left(S_{1}>t, S_{2}>t\right)$. Logo

$$
\begin{aligned}
I^{\Phi}(\{12\}) & =E\left[\int_{0}^{\infty} I\left(0<s \leq S_{1} \wedge S_{2}\right) d A_{\{12\}}(s)\right] \\
& =E\left[\int_{0}^{\infty} I\left(0<s \leq S_{1} \wedge S_{2}\right) \beta_{12} I\left(S_{1}>s, S_{2}>s\right) d s\right] \\
& =\beta_{12} E\left[S_{1} \wedge S_{2}\right] \\
& =\frac{\beta_{12}}{\beta_{1}+\beta_{2}+\beta_{12}} .
\end{aligned}
$$

Se considerarmos um sistema em paralelo, com tempo de vida $T=\Phi\left(S_{1}, S_{2}\right)=$ $S_{1} \vee S_{2}$, o nível crítico para o bloco $\{2\}$ é $Y_{\Phi}(\{2\})=Z_{1}$ e seu compensador é

$$
A_{\{2\}}(t)=\beta_{2} t I\left(S_{1}>t, S_{2}>t\right)+\left(\beta_{2}+\beta_{12}\right) t I\left(S_{1} \leq t, S_{2}>t\right) .
$$

Portanto

$$
\begin{aligned}
I^{\Phi}(\{2\}) & =E\left[\int_{0}^{\infty} I\left(Z_{1}<s \leq S_{1} \vee S_{2}\right) d A_{\{2\}}(s)\right] \\
& =E\left[\int _ { 0 } ^ { \infty } I ( Z _ { 1 } < s \leq S _ { 1 } \vee S _ { 2 } ) \left[\beta_{2} I\left(S_{1}>s, S_{2}>s\right)\right.\right. \\
& \left.\left.+\left(\beta_{2}+\beta_{12}\right) I\left(S_{1} \leq s, S_{2}>s\right)\right] d s\right] \\
& \left.=E\left[\int_{0}^{\infty} I\left(Z_{1}<s \leq Z_{2} \wedge Z_{12}\right)\left(\beta_{2}+\beta_{12}\right)\right] d s\right] \\
& =1-\frac{\beta_{2}+\beta_{12}}{\beta_{1}+\beta_{2}+\beta_{12}} .
\end{aligned}
$$


Seguindo o mesmo raciocínio, obtemos

$$
I^{\Phi}(\{1\})=1-\frac{\beta_{1}+\beta_{12}}{\beta_{1}+\beta_{2}+\beta_{12}},
$$

e pelo fato de que as somas das medidas de importâncias é igual a $1\left(I^{\Phi}(\{1\})+\right.$ $\left.I^{\Phi}(\{2\})+I^{\Phi}(\{12\})=1\right)$, temos que:

$$
I^{\Phi}(\{12\})=\frac{\beta_{12}}{\beta_{1}+\beta_{2}+\beta_{12}} .
$$

Exemplo 1.15. Seja $T=S_{1} \wedge\left(S_{2} \vee S_{3}\right)$ onde os $S_{i}$ são tempos de vidas independentes e $S_{i} \sim \operatorname{Weibull}\left(\beta_{i}, \alpha_{i}\right), \beta_{i}>1, \alpha_{i}>0, i=1,2,3$,são os parâmetros de forma e de escalas respectivamente. Como os tempos de vidas dos componentes são independentes(não existem falhas multiplas) então os unicos blocos são $\{i\}, i=1,2,3$ e seus $\Im_{t^{-}}$ compensadores coincidem com os compensadores dos processos $I\left(S_{i} \leq t\right), i=1,2,3$ onde $\Im_{t}=\sigma\left\{I\left(S_{i} \leq s\right): 0 \leq s \leq t, i=1,2,3\right\}$ os quais são,

$$
A_{\{i\}}(t)=A_{i}(t)=-\ln \left(\bar{F}_{i}\left(S_{i} \wedge t\right)\right)=\left(\frac{t \wedge S_{i}}{\alpha_{i}}\right)^{\beta_{i}}, i=1,2,3 .
$$

Observando a função estrutura do sistema $\left(T=\Phi\left(S_{1}, S_{2}, S_{3}\right)=S_{1} \wedge\left(S_{2} \vee S_{3}\right)\right)$ obtemos os níveis críticos para os blocos que são $Y_{\Phi}(\{1\})=0, Y_{\Phi}(\{2\})=S_{3} e$ $Y_{\Phi}(\{3\})=S_{2}$ segue-se então da definição 1.10 que as medidas de importâncias são:

$$
\begin{aligned}
& I^{\Phi}(\{1\})=E\left[\left(\frac{T \wedge S_{1}}{\alpha_{1}}\right)^{\beta_{1}}\right], \\
& I^{\Phi}(\{2\})=E\left[\left(\frac{T \wedge S_{2}}{\alpha_{2}}\right)^{\beta_{2}}-\left(\frac{S_{3} \wedge S_{2}}{\alpha_{2}}\right)^{\beta_{2}}\right]^{+} \\
& I^{\Phi}(\{3\})=E\left[\left(\frac{T \wedge S_{3}}{\alpha_{3}}\right)^{\beta_{3}}-\left(\frac{S_{3} \wedge S_{2}}{\alpha_{3}}\right)^{\beta_{3}}\right]^{+} .
\end{aligned}
$$

Exemplo 1.16. Seja $\Phi$ um sistema $k$-de-n, isto é:

$$
\Phi\left(\mathbf{X}_{t}\right)= \begin{cases}1 & \text { se } \sum_{i}^{n} X_{t}(i) \geq k \\ 0 & \text { caso contrario }\end{cases}
$$

onde $\mathrm{X}_{t}=\left(X_{t}(1), \ldots, X_{t}(n)\right), X_{t}(i)=I\left(S_{i}>t\right)$ e $S_{i}, i=1, \ldots, n$ são os tempos de vidas dos componentes, com funções de distribuiçõs absolutamente contínuas. 
Neste caso, para todo $i=1, \ldots, n$ os níveis críticos para os blocos $\{i\}, i=1, \ldots, n$ são todos iguais e coincidem com o tempo da ( $n-k$ )-ésima falha (ou tempo que tem exatamente $k$ componentes funcionando), isto é, $Y_{\Phi}(\{i\})=T_{(n-k)}, i=1, \ldots, n$, e o tempo de vida do sistema $T=\Phi\left(S_{1}, \ldots, S_{1}\right)=T_{(n-k+1)}$.

As medidas de importância dos blocos $\{i\}$ são dadas por

$$
\begin{aligned}
I^{\Phi}(\{i\}) & =E\left[A_{\{i\}}\left(T_{(n-k+1)}\right)-A_{\{i\}}\left(T_{(n-k)}\right)\right]^{+} \\
& =E\left[A_{\{i\}}\left(T_{(n-k+1)}\right)\right]-E\left[A_{\{i\}}\left(T_{(n-k)}\right)\right], i=1, \ldots, n .
\end{aligned}
$$

Se os $S_{i}$ são identicamente distribuidos, então $E A_{\{i\}}(t)=E A_{\{j\}}(t)$ para todo $t \geq$ $0,1 \leq i, j \leq n$, e portanto $I^{\Phi}(\{i\})=1 / n, i=1, \ldots, n$.

\subsection{Comparando medidas de importâncias através de transformações nos compensadores}

Comentamos na Seção 1.2 que a medida de importância da confiabilidade dos componentes esta relacionada diretamente com a confiabilidade do sistema. Se os componentes são independentes esta relação é:

$$
\begin{aligned}
P(T \leq t) & =\sum_{i=1}^{n} \int_{0}^{t} I^{B}(i, s) d F_{i}(s) \\
& =\sum_{i=1}^{n} I^{B P}(i, t)
\end{aligned}
$$

onde $I^{B}(i, s)$ é a medida de importância da confiabilidade do componente $i$ para a confiabilidade do sistema, de Birnbaum, $F_{i}(s)$ é a função de distribuição do seu tempo de vida $S_{i}$ e $I^{B P}(i, t)$ é importância da confiabilidade de Barlow e Proschan do componente $i$ até o instante $t$.

Segue que se modificamos a função de distribuição do componente $i$, digamos de $F_{i}(s)$ para $G_{i}(s)$, e não alteramos as distribuições dos outros componentes, esta 
relacão (1.17) transforma-se em

$$
P(T \leq t)=\sum_{j \neq i} \int_{0}^{t} I^{B}(j, s) d F_{j}(s)+\int_{0}^{t} I^{B}(i, s) d G_{i}(s) .
$$

Se $G_{i}(s) \leq F_{i}(s)$, isto é, o componente $i$ torna-se "estocásticamente melhor,"e se $I^{B}(i, s)$ é uma função decrescente em $s$, melhoramos a confiabilidade do sistema através dessa transformação.

Queremos estender estas transformações para o caso onde existe dependência entre os componentes mas que não admita falhas multiplas, isto é:

Considere um sistema coerente $\Phi$, com tempo de vida $T$ e $n$ componentes $C_{1}, C_{2}$, $\ldots, C_{n}$. Cada componente $C_{i}$, tem um tempo de vida aleatório $S_{i}, i=1, \ldots, n$, positivos, definidos em um mesmo espaço de probabilidade completo $(\Omega, \Im, P)$. Assumimos que os componentes são dependentes mas com falhas distintas, isto é, $P\left(S_{i} \neq S_{j}\right)=1$, para $i \neq j$. A formulação matemática de nossas observação são através da familia de sub- $\sigma$-àlgebra $\left(\Im_{t}\right)_{t \geq 0}$ onde

$$
\Im_{t}=\sigma\left\{I\left(S_{i}>s\right), i=1,2, \ldots, n, s \leq t\right\} .
$$

Assumimos que $S_{i}, i=1, \ldots, n$, são $\Im_{t}$-tempos de parada totalmente inacessíveis de forma que os $\Im_{t}$-compensadores $A_{i}(t)=A_{\{i\}}(t)$ correspondentes aos processos $\left(N_{i}(t)\right)=\left(I\left(S_{i} \leq t\right)\right)_{t \geq 0}$ tem a representação $A_{i}(t)=\int_{0}^{t} I\left(S_{i}>s\right) \lambda_{i}(s) d s, i=$ $1, \ldots, n$, onde $\lambda_{i}(s)$ é a $\Im_{s}$-intensidade previsível.

Algumas propriedades clássicas para a importância de componentes podem ser estendidas em nosso contexto, tais como:

Proposição 1.17. Suponhamos que os tempos de vidas dos componentes de um sistema $\Phi, S_{i}, i=1, \ldots, n$, tem distribuições $F_{i}, i=1, \ldots, n$ absolutamente contínuas 
e que o i-ésimo componente está em série com o resto do sistema. Se $F_{i}(t) \geq$ $F_{j}(t), j \neq i$, para todo $t \geq 0$, então

$$
I^{\Phi}(\{i\}) \geq I^{\Phi}(\{j\})
$$

para todo $j \neq i$.

Prova: Como os tempos de vidas dos componentes são absolutamente contínuos então $P\left(S_{i}=S_{j}\right)=0$ se $j \neq i$, isto é, a probabilidade de existir falhas multiplas é nula, portanto $\{j\}, j=1, \ldots, n$ são os unicos blocos e $S_{j}=T_{\{j\}}, j=1, \ldots, n$. Seja $A_{j}(t)$ o $\Im_{t}$-compensador do processo $N_{\{j\}}(t)=I\left(S_{j} \leq t\right), j=1, \ldots, n$ onde $\Im_{t}$ é as historia dos componentes até o instante $t$. Segue-se de (1.9) que $F_{j}(t)=E A_{j}(t)$ para todo $t \geq 0$ e para todo $j=1, \ldots, n$, e por hipótese $E A_{i}(t) \geq E A_{j}(t), j \neq i$. Como o componente $i$ está em serie com o resto do sistema então o seu nível crítico $Y_{\Phi}(\{i\})=0$ e portanto $I^{\Phi}(\{i\})=E A_{i}(T)$, onde $T$ é o tempo de vida do sistema. Segue-se dos argumentos acima e da Definição 1.10 que

$$
I^{\Phi}(\{i\})=E A_{i}(T) \geq E A_{j}(T) \geq E\left[A_{j}(T)-A_{j}\left(Y_{\Phi}(\{j\})\right)\right]^{+}=I^{\Phi}(\{j\}),
$$

para todo $j \neq i$.

Proposição 1.18. Suponhamos que os tempos de vidas dos componentes de um sistema $\Phi, S_{i}, i=1, \ldots, n$, tem distribuições $F_{i}, i=1, \ldots, n$ absolutamente contínuas e que o i-ésimo componente está em paralelo com o resto do sistema. Se $F_{i}(t) \leq$ $F_{j}(t), j \neq i$, para todo $t \geq 0$, e $P\left(S_{i} \leq Y_{\Phi}(i)\right) \leq P\left(S_{j} \leq Y_{\Phi}(j)\right), j \neq i$, então

$$
I^{\Phi}(\{i\}) \geq I^{\Phi}(\{j\}),
$$


para todo $j \neq i$.

Prova: Pela hipótese de que os tempos de vidas dos componentes que compõem o sistema $\Phi$ tem distribuições a.c., os unicos blocos existente são da forma $\{j\}, j=$ $1,2, \ldots, n$ e os seus respectivos tempos de vida $T_{\{j\}}=S_{j} P$-q.c. e portanto não existem distinção entre os tempos de vidas dos componente $j, j=1, \ldots, n$ e os tempos de vidas dos blocos $\{j\}, j=1, \ldots, n$. Seja $\Im_{t}$ a pré-t-história dos componentes $e$ $A_{j}(t), j=1, \ldots, n$ os $\Im_{t}$-compensadores dos processos $I\left(S_{j} \leq t\right), j=1, \ldots, n$.

Pelo fato de que $F_{i}(t) \leq F_{j}(t), j \neq i$, para todo $t \geq 0$, e pela equação (1.9) temos $E A_{i}(t) \leq E A_{j}(t)$ para todo $t \geq 0$, e $j \neq i$. Como o componente $i$ está em paralelo com o resto do sistema, temos $S_{i} \leq T$, onde $T$ é o tempo de vida do sistema. Usando a hipótese de que $P\left(S_{i} \leq Y_{\Phi}(i)\right) \leq P\left(S_{j} \leq Y_{\Phi}(j)\right), j \neq i$, para $j \neq i$, e o fato de que o $\Im_{t}$-compensador $A_{i}(t)$ no seu tempo final $S_{i}$ é uma v.a. com distribuiçãa exponencial de média 1 (veja Proposição 1.7), segue-se que

$$
\begin{aligned}
I^{\Phi}(\{i\}) & =E\left[A_{i}(T)-A_{i}\left(Y_{\Phi}(i)\right)\right]^{+}=E\left[A_{i}\left(S_{i}\right)-A_{i}\left(Y_{\Phi}(i)\right)\right]^{+} \\
& =E\left[A_{i}\left(S_{i}\right)\right]-E\left[A_{i}\left(Y_{\Phi}(i)\right)\right]=1-E\left[A_{i}\left(Y_{\Phi}(i)\right)\right] \\
& \geq 1-E\left[A_{j}\left(Y_{\Phi}(j)\right)\right] \geq E\left[A_{j}(T)-A_{j}\left(Y_{\Phi}(j)\right)\right]^{+}=I^{\Phi}(\{j\}),
\end{aligned}
$$

para $j \neq i$. Note que a desigualdade acima é devido a hipótese $P\left(S_{i} \leq Y_{\Phi}(i)\right) \leq$ $P\left(S_{j} \leq Y_{\Phi}(j)\right)$ e por consequência $E\left[A_{i}\left(Y_{\Phi}(i)\right)\right] \leq E\left[A_{j}\left(Y_{\Phi}(j)\right)\right]$.

Observe que pelas condições estabelecidas acima temos:

$$
E[I(T \leq t)]=P(T \leq t)=\sum_{i=1}^{n} E\left[\int_{Y_{\Phi}(i)}^{t \wedge T} d A_{i}(s)\right]^{+},
$$




\subsection{Comparando medidas de importâncias através de transformações}

nos compensadores

onde $E\left[\int_{Y_{\Phi}(i)}^{t \wedge T} d A_{i}(s)\right]^{+}$é uma extensão da medida de importância de Barlow e Proschan do componente $i$ no intervalo $[0, t]$ a qual denotaremos por $I^{\Phi}(i, t)$.

Neste caso a melhora da confiabilidade do sistema é descrita por meio de transformações dos seus compensadores.

Desenvolveremos a idéia através de um exemplo onde consideramos a transformação por meio de um reparo mínimo

Definição 1.19. (Reparo Mínimo)

O reparo mínimo de um componente $i$ é um reparo que reconstitui a sua intensidade de falha, ou seu compensador, ao instante imediatamente anterior à falha.

No caso de independência o reparo mínimo do componente $i$ transforma a sua função de sobrevivência $\bar{F}_{i}(t)=1-F_{i}(t)$ para $\bar{G}_{i}(t)=\bar{F}_{i}(t)-\bar{F}_{i}(t) \ln \left(\bar{F}_{i}(t)\right)$.

No caso de componentes dependentes o reparo mínimo do componente $i$ gera uma modificação do compensador $A_{i}(t)$ para, digamos, $B_{i}(t)$.

Intuitivamente, interpretamos que $S_{i}$ é o tempo em que a função crescente $A_{i}(t)$ atinge um tempo exponencial $X$ de parâmetro 1. Pela propriedade da falta de memória da distribuição exponencial, podemos ignorar a primeira falha do componente $i$ trocando a distribuição exponencial de parâmetro 1 por uma distribuição Gama de parâmetro $(2,1)$. Enfim a distribuição Gama $(2,1)$ é a soma de duas variáveis aleatórias independentes com distribuições Exponenciais de média 1, que podem ser pensadas como os valores determinando a primeira falha ignorada e o tempo de vida adicional devido ao reparo mínimo.

Note também que a função de risco da distribuição Gama $(2,1)$ é $R(t)=-\ln (\bar{F}(t))$ 
$=t-\ln (1+t)$. Se procurarmos o novo compensador $B_{i}(t)$ que atinja um tempo exponencial em $S_{i}+S_{i}^{\prime}$, onde $S_{i}$ é o tempo de vida original do componente $i$ e $S_{i}^{\prime}$ é o tempo de vida adicional devido ao reparo mínimo, observamos que $A_{i}(t)=X$ é equivalente a $R\left(A_{i}(t)\right)=R(X)$. Se $X$ tem distribuição Gamma $(2,1), R(X)$ é exponencial de parâmetro 1 , e o compensador procurado é

$$
B_{i}(t)=R\left(A_{i}(t)\right)=A_{i}(t)-\ln \left(1+A_{i}(t)\right)
$$

É claro que pelo fato da decomposição de Doob-Meyer ser única esta transformação ocorre através de uma modificação na medida de probabilidade $P$. Os resultados que veremos a seguir são casos particulares do teorema de Girsanov (veja Teorema $A .16$ e suas observações).

Inicialmente consideremos a medida

$$
Q_{i}(A)=\int_{A} A_{i}\left(S_{i}\right) d P
$$

$A \in \Im$, que define a derivada de Radon-Nikodym $\frac{d Q_{i}}{d P}=A_{i}\left(S_{i}\right)$. Norros (1986b) demonstra que $Q_{i}$ é a medida obtida de $P$ através do reparo mínimo do componente $i$ e $B_{i}(t)=A_{i}(t)-\ln \left(1+A_{i}(t)\right)$ é o $\Im_{t}$-compensador do processo $I\left(S_{i} \leq t\right)$ sob esta medida. Para $j \neq i$ prova-se que $B_{j}(t)=A_{j}(t)$, uma vez que o reparo é feito apenas no componente $i$.

Proposição 1.20. (Norros (1986b))

Seja $\Phi$ um sistema coerente composto de $n$ componentes cujos os tempos de vidas satisfazem as condições descritas nesta seção. Se

$$
L_{t}=E_{P}\left[\frac{d Q_{i}}{d P} \mid \Im_{t}\right]=E_{P}\left[A_{i}\left(S_{i}\right) \mid \Im_{t}\right]=1-M_{i}(t)=1-N_{i}(t)+A_{i}(t)
$$




$$
B_{i}(t)=A_{i}(t)-\ln \left(1+A_{i}(t)\right)
$$

e

$$
B_{j}(t)=A_{j}(t)
$$

se $j \neq i$.

Então o $Q_{i}$-compensador de $N_{i}(t)$ é $B_{i}(t), i=1, \ldots, n$.

Segue do teorema seguinte que a transformação do tempo de vida $S_{i}$ através de um reparo mínimo produz um tempo de vida estocásticamente melhor.

Teorema 1.21. Seja $\frac{d Q_{i}}{d P}=A_{i}\left(S_{i}\right)$, onde $S_{i}$ é o tempo de vida do componente $i$, então $P\left(S_{i}>t\right) \leq Q_{i}\left(S_{i}>t\right)$ para todo $t \geq 0$.

Prova: Note que $L_{t}=E_{P}\left[A_{i}\left(S_{i}\right) \mid \Im_{t}\right]=I\left(S_{i}>t\right)+A_{i}(t)$, além disso, $P\left(S_{i} \leq\right.$ $t)=\int_{\left\{S_{i} \leq t\right\}} d P=E_{P}\left[A_{i}(t)\right]$ e $Q_{i}\left(S_{i} \leq t\right)=\int_{\left\{S_{i} \leq t\right\}} d Q_{i}=E_{Q_{i}}\left[B_{i}(t)\right]$.

Assim

$$
\begin{aligned}
Q_{i}\left(S_{i} \leq t\right) & =\int_{\left\{S_{i} \leq t\right\}} d Q_{i}=\int_{\left\{S_{i} \leq t\right\}} L_{t} d P=\int_{\left\{S_{i} \leq t\right\}} A_{i}(t) d P \\
& <\int_{\Omega} A_{i}(t) d P=E_{P}\left[A_{i}(t)\right]=P\left(S_{i} \leq t\right)
\end{aligned}
$$

Além disso, com a condição $\operatorname{Cov}_{P}\left(L_{t}, A_{j}(t)\right) \leq 0$, para $j \neq i$, essa mesma transformação produz melhoras estocásticas nos outros componentes.

Teorema 1.22. Se $\operatorname{Cov}_{P}\left(L_{t}, A_{j}(t)\right) \leq 0$ para $j \neq i$ então $P\left(S_{j}>t\right) \leq Q_{i}\left(S_{j}>t\right)$. 
Prova: Como por hipótese $\operatorname{Cov}_{P}\left(L_{t}, A_{j}(t)\right) \leq 0$, temos $E_{P}\left[L_{t} A_{j}(t)\right] \leq E_{P}\left[A_{j}(t)\right]$ um vez que $L_{t}$ é um $\left(P, \Im_{t}\right)$-martingal de média 1 . Assim:

$$
Q_{i}\left(S_{j} \leq t\right)=E_{Q_{i}}\left[A_{j}(t)\right]=E_{P}\left[A_{j}(t) L_{t}\right] \leq E_{P}\left[A_{j}(t)\right]=P\left(S_{j} \leq t\right)
$$

Usando a definição de importância 1.10 e trocando os compensadores $A_{i}(t)$ por $B_{i}(t)$ e a medida $P$ pela medida $Q_{i}$, pode-se definir a medida de importância do componente $i$ através de reparos mínimos por:

$$
\begin{aligned}
I^{1}(i) & =E_{Q_{i}}\left[\left(B_{i}(T)-B_{i}\left(Y_{\Phi}(i)\right)\right)^{+}\right] \\
& =E_{Q_{i}}\left[\int_{0}^{\infty} I\left(Y_{\Phi}(i)<t \leq T\right) d B_{i}(t)\right] \\
& =E_{Q_{i}}\left[\int_{0}^{\infty} I\left(Y_{\Phi}(i)<t \leq T\right) \frac{A_{i}(t)}{1+A_{i}(t)} d A_{i}(t)\right] \\
& =E_{P}\left[A_{i}\left(S_{i}\right) \int_{0}^{\infty} I\left(Y_{\Phi}(i)<t \leq T\right) \frac{A_{i}(t)}{1+A_{i}(t)} d A_{i}(t)\right],
\end{aligned}
$$

onde $T$ é o tempo de vida do sistema e $Y_{\Phi}(i)$ o nível crítico do componente $i$.

A medida de importância para os componentes $j \neq i$ com respeito a medida $Q_{i}$ é dada por

$$
\begin{aligned}
I^{1}(j) & =E_{Q_{i}}\left[\left(B_{j}(T)-B_{i}\left(Y_{\Phi}(j)\right)\right)^{+}\right] \\
& =E_{Q_{i}}\left[\int_{0}^{\infty} I\left(Y_{\Phi}(j)<t \leq T\right) d B_{j}(t)\right] \\
& =E_{Q_{i}}\left[\int_{0}^{\infty} I\left(Y_{\Phi}(j)<t \leq T\right) d A_{j}(t)\right] \\
& =E_{P}\left[A_{i}\left(S_{i}\right) \int_{0}^{\infty} I\left(Y_{\Phi}(i)<t \leq T\right) d A_{i}(t)\right] .
\end{aligned}
$$

Também podemos calcular a medida de importância de Barlow e Proschan do componente $j=1, \ldots, n$, no intervalo $[0, t]$ por

$$
I^{1}(j, t)=E_{Q_{i}}\left[\left(B_{j}(t \wedge T)-B_{j}\left(Y_{\Phi}(j)\right)\right)^{+}\right]
$$


onde $T$ é o tempo de vida do sistema.

Observação 1.23. Aplicando o Teorema 1.6, temos

i) $Q_{i}(T \leq t)=\sum_{j=1}^{n} I^{1}(j, t)$,

ii) $\sum_{j=1}^{n} I^{1}(j)=1$.

Teorema 1.24. Se $T=\Phi\left(S_{1}, \ldots, S_{n}\right)=\min \left\{S_{1}, \ldots, S_{n}\right\}, \operatorname{Cov}_{P}\left(L_{t}, A_{j}(t)\right) \leq 0$, para todo $j=1,2, \ldots, n$ e para todo $t \geq 0$, então

$$
Q_{i}(T>t) \geq P(T>t)
$$

e conseqüentemente, $E_{P}[T] \leq E_{Q_{i}}[T]$.

Prova: Como $\Phi$ é um sistema em série, os níveis críticos dos componentes $C_{j}, j=1, \ldots, n$. é zero e então as probabilidades (com respeito a $P$ e $Q_{i}$ ) de $T$ falhar em $[0, t]$ podem ser obtidas da seguinte maneira,

$$
\begin{aligned}
P(T \leq t) & =\sum_{j=1}^{n} E_{P}\left[A_{j}(t \wedge T)\right], \\
Q_{i}(T \leq t) & =\sum_{j=1}^{n} E_{Q_{i}}\left[B_{j}(t \wedge T)\right],
\end{aligned}
$$

onde os $Q_{i}$-compensadores são $B_{i}(t)=A_{i}(t)-\ln \left(1+A_{i}(t)\right)$ e $B_{j}(t)=A_{j}(t), j \neq i$. Sendo assim temos

$$
\begin{aligned}
P(T \leq t)-Q_{i}(T \leq t) & =\sum_{j=1}^{n} E_{P}\left[A_{j}(t \wedge T)\right]-\sum_{j=1}^{n} E_{Q_{i}}\left[B_{j}(t \wedge T)\right] \\
& =\sum_{j=1}^{n} E_{P}\left[A_{j}(t \wedge T)\right]-\sum_{j=1}^{n} E_{P}\left[L_{t \wedge T} A_{j}(t \wedge T)\right] \\
& +E_{Q_{i}}\left[\ln \left(1+A_{i}(t)\right)\right] \\
& =\sum_{j=1}^{n} E_{P}\left[\left(1-L_{t \wedge T}\right) A_{j}(t \wedge T)\right]+E_{Q_{i}}\left[\ln \left(1+A_{i}(t)\right)\right] .
\end{aligned}
$$


No entanto, $1-L_{t}=M_{i}(t)$ e portanto $\operatorname{Cov}_{P}\left(L_{t}, A_{j}(t)\right) \leq 0$, se e somente se $\operatorname{Cov}_{P}\left(M_{i}(t), A_{j}(t)\right)=-\operatorname{Cov}_{P}\left(L_{t}, A_{j}(t)\right) \geq 0$, além disso, $E_{Q_{i}}\left[\ln \left(1+A_{i}(t)\right] \geq 0\right.$, pois $A_{i}(t) \geq 0$ para todo $t \geq 0$, logo podemos concluir que $P(T \leq t)-Q_{i}(T \leq t) \geq 0$ e conseqüentemente $Q_{i}(T>t)-P(T>t) \geq 0$.

Como resultado importante por si mesmo temos que sob a condição de que $\operatorname{Cov}_{P}\left(L_{t}, A_{j}(t)\right) \leq 0$ a associação entre os componentes sob a medida $P$ é preservada sob a medida $Q_{i}$.

Teorema 1.25. Se $\operatorname{Cov}_{P}\left(I\left(S_{i} \leq t\right), I\left(S_{j} \leq t\right)\right) \geq 0$ e $\operatorname{Cov}_{P}\left(L_{t}, A_{j}(t)\right) \leq 0$, então $\operatorname{Cov}_{Q_{i}}\left(I\left(S_{i} \leq t\right), I\left(S_{j} \leq t\right)\right) \geq 0$.

Prova: Segue da definição de covariância e usando o fato de que $L_{t}=1-M_{i}(t)$ temos:

$$
\begin{aligned}
\operatorname{Cov}_{Q_{i}}\left(I\left(S_{i} \leq t\right), I\left(S_{j} \leq t\right)\right) & =E_{Q_{i}}\left[I\left(S_{i} \leq t\right) I\left(S_{j} \leq t\right)\right] \\
& -E_{Q_{i}}\left[I\left(S_{i} \leq t\right)\right] E_{Q_{i}}\left[I\left(S_{j} \leq t\right)\right] \\
& =E_{P}\left[\left(1-M_{i}(t)\right) I\left(S_{i} \leq t\right) I\left(S_{j} \leq t\right)\right] \\
& -E_{P}\left[\left(1-M_{i}(t)\right) I\left(S_{i} \leq t\right)\right] E_{P}\left[\left(1-M_{i}(t)\right) I\left(S_{j} \leq t\right)\right] \\
& =\operatorname{Cov}\left(I\left(S_{i} \leq t\right), I\left(S_{j} \leq t\right)\right) \\
& +\{\underbrace{\left.E_{P}\left[M_{i}(t)\right) I\left(S_{j} \leq t\right)\right]}_{I I I} \\
& \times \underbrace{\left.\left[E_{P}\left[I\left(S_{i} \leq t\right)\right]-E_{P}\left[M_{i}(t)\right) I\left(S_{i} \leq t\right)\right]\right]}_{I I}\} \\
& +\underbrace{\left.E_{P}\left[I\left(S_{j} \leq t\right)\right] E_{P}\left[M_{i}(t)\right) I\left(S_{i} \leq t\right)\right]}_{I I} .
\end{aligned}
$$


Como

$$
\begin{aligned}
\left.E_{P}\left[M_{i}(t)\right) I\left(S_{i} \leq t\right)\right] & =E_{P}\left[\left(I\left(S_{i} \leq t\right)-A_{i}(t)\right) I\left(S_{i} \leq t\right)\right] \\
& =E_{P}\left[I\left(S_{i} \leq t\right)\right]-E_{P}\left[A_{i}(t) I\left(S_{i} \leq t\right)\right] \\
& \geq E_{P}\left[I\left(S_{i} \leq t\right)\right]-E_{P}\left[A_{i}(t)\right]=0
\end{aligned}
$$

e pelo fato de que $E_{P}\left[A_{i}(t) I\left(S_{i} \leq t\right)\right] \geq 0$, temos que $I I \geq 0$ e III $\geq 0$. Para mostrarmos que $I \geq 0$, basta observarmos que

$$
\begin{aligned}
\left.E_{P}\left[M_{i}(t)\right) I\left(S_{j} \leq t\right)\right] & =E_{Q_{i}}\left[\frac{M_{i}(t)}{L_{t}} I\left(S_{j} \leq t\right)\right] \\
& =E_{Q_{i}}\left[\frac{1-L_{t}}{L_{t}} I\left(S_{j} \leq t\right)\right] \\
& =E_{Q_{i}}\left[\frac{I\left(S_{j} \leq t\right)}{L_{t}}\right]-E_{Q_{i}}\left[I\left(S_{j} \leq t\right)\right] \\
& =E_{P}\left[I\left(S_{j} \leq t\right)\right]-E_{Q_{i}}\left[I\left(S_{j} \leq t\right)\right] \geq 0,
\end{aligned}
$$

onde a ultima desigualdade é pela hipótese de que $\operatorname{Cov}_{P}\left(L_{t}, A_{j}(t)\right) \leq 0$ e aplicamos o Teorema 1.22, portanto o teorema está provado.

\subsection{Importância de um Módulo}

Vamos considerar uma função de estrutura $\Phi$ representada pela decomposição série-paralelo

$$
\Phi\left(\mathrm{X}_{t}\right)=\min _{1 \leq j \leq k} \max _{i \in K_{j}} X_{t}(i)
$$

onde $K_{j}, j=1, \ldots, k$ são os conjuntos de cortes mínimais e

$$
\mathrm{X}_{t}=\left(X_{t}(1), \ldots, X_{t}(n)\right), X_{t}(i)=I\left(S_{i}>t\right)
$$


Intuitivamente, um módulo de um sistema coerente é um subconjunto de componentes que consideramos como um "supercomponente". Técnicamente a estrutura coerente $\Phi$ de $n$ componentes pode ser representada por

$$
\Phi\left(\mathbf{X}_{t}\right)=\Psi\left[\left(\chi\left(\mathbf{X}_{t}(M)\right), \mathbf{X}_{t}\left(M^{c}\right)\right)\right]
$$

onde $M$ é um subconjunto de $C=\{1, \ldots, n\}$ com complementar $M^{c}=C-M, \mathrm{X}_{t}(M)$ é um vetor cujas as componentes são da forma $I\left(S_{i}>t\right), i \in M, \chi$ é uma função de estrutura de um subsistema de $\Phi$ e $\Psi$ é uma estrutura de organização. Em geral a notação $(M, \chi)$ representa um módulo de $\Phi$ e $\Psi$ é visto com um sistema coerente composto pelos componentes $(M, \chi)$ ( um supercomponente) e $j \in M^{c}$. Neste contexto faz sentido perguntar sobre a importância da confiabilidade do módulo (supercomponente) para a confiabilidade do sistema.

Um resultado clássico (veja Barlow e Proschan (1975)) é que sob a hipótese de que os tempos de vidas dos componentes que compõem o sistema $\Phi$ sejam independentes e tenham distribuições absolutamente contínuas temos

$$
I^{\Psi}(\chi)=\sum_{i \in M} I^{B P}(i)
$$

onde $I^{B P}(i)$ é a importância de Barlow e Proschan do componente $i$ para o sistema $\Phi$. Queremos provar que este resultado se estende para o caso de dependência entre os componentes, utilizando o conceito de medidas de importâncias para blocos definida em 1.10, ou seja, provaremos que

$$
I^{\Psi}(\chi)=\sum_{\emptyset \neq L \subset M} I^{\Phi}(L)
$$

onde $I^{\Phi}(L)$ é medida de importância do bloco $L$ para o sistema $\Phi$, segundo a Definição 1.10 .

Segue de (1.27) que o tempo de vida $T$ do sistema $\Phi$ pode ser escrito como

$$
T=\inf \left\{t: \Psi\left[\left(\chi\left(\mathbf{X}_{t}(M)\right), \mathbf{X}_{t}\left(M^{c}\right)\right)\right]=0\right\},
$$


e o tempo de vida do módulo $\chi, T_{\chi}$, como

$$
T_{\chi}=\inf \left\{t: \chi\left(\mathbf{X}_{t}(M)\right)=0\right\}
$$

Se $A_{\chi}(t)$ é o $\Im_{t}$-compensador do processo $I\left(T_{\chi} \leq t\right)$ calculamos a importância de $\chi$ para o sistema $\Psi$ (veja Definição 1.10)

$$
\begin{aligned}
I^{\Psi}(\chi) & =E\left[A_{\chi}(T)-A_{\chi}\left(Y_{\Psi}(\chi)\right)\right]^{+} \\
& =E\left[\int_{0}^{\infty} I\left(Y_{\Psi}(\chi)<t \leq T\right) d A_{\chi}(t)\right],
\end{aligned}
$$

onde $Y_{\Psi}(\chi)$ é o nível crítico do módulo $\chi$ para a estrutura $\Psi$.

Como o módulo $(M, \chi)$ é um subsistema de $\Phi$, cujo tempo de vida é justamente $T_{\chi}$, a medida de importância para os blocos $L \subseteq M$, para $\chi$ é

$$
\begin{aligned}
I^{\chi}(L) & =E\left[A_{L}\left(T_{\chi}\right)-A_{L}\left(Y_{\chi}(L)\right)\right]^{+} \\
& =E\left[\int_{0}^{\infty} I\left(Y_{\chi}(L)<t \leq T_{\chi}\right) d A_{L}(t)\right]
\end{aligned}
$$

onde $Y_{\chi}(L)$ é o nível crítico do bloco $L$ para o sistema $\chi$. Pelo Teorema 1.6 temos

$$
\begin{aligned}
A_{\chi}(t) & =\sum_{\emptyset \neq L \subset M} \int_{0}^{\infty} I\left(Y_{\chi}(L)<s \leq T_{\chi} \wedge t\right) d A_{L}(s) \\
& =\sum_{\emptyset \neq L \subset M}\left[A_{L}\left(T_{\chi} \wedge t\right)-A_{L}\left(Y_{\chi}(L)\right)\right]^{+} .
\end{aligned}
$$

Para provarmos que $I^{\Psi}(\chi)=\sum_{\emptyset \neq L \subset M} I^{\Phi}(L)$, consideremos a seguinte proposição:

Proposição 1.26. Seja $(M, \chi)$ um módulo de um sistema coerente $\Phi$, com estrutura de organização $\Psi$ e seja $L \subset M$. Então o bloco $L$ é crítico para o sistema $\Phi$ no instante $t$ se e somente se $L$ é crítico para o módulo $(M, \chi)$ e o módulo é crítico 
para estrutura de organização $\Psi$ no instante $t$, isto é,

$$
\left\{\Phi\left(1_{L}, \mathbf{X}_{t}\right)-\Phi\left(0_{L}, \mathbf{X}_{t}\right)=1\right\}=A \cap B
$$

onde,

$$
A=\left\{\Psi\left(1, \mathbf{X}_{t}\left(M^{c}\right)\right)-\Psi\left(0, \mathbf{X}_{t}\left(M^{c}\right)\right)=1\right\}
$$

$e$

$$
B=\left\{\chi\left(\mathbf{1}_{L}, \mathbf{X}_{t}(M)\right)-\chi\left(\mathbf{0}_{L}, \mathbf{X}_{t}(M)\right)=1\right\}
$$

\section{Prova:}

Observe que, para todo $L \neq \emptyset, L \subset M$, temos

$$
\begin{aligned}
\Phi\left(1_{L}, \mathbf{X}_{t}\right)-\Phi\left(\mathbf{0}_{L}, \mathbf{X}_{t}\right) & =\Psi\left(\chi\left(\mathbf{1}_{L}, \mathbf{X}_{t}(M)\right), \mathbf{X}_{t}\left(M^{c}\right)\right) \\
& -\Psi\left(\chi\left(\mathbf{0}_{L}, \mathbf{X}_{t}(M)\right), \mathbf{X}_{t}\left(M^{c}\right)\right) \\
& =\chi\left(\mathbf{1}_{L}, \mathbf{X}_{t}(M)\right) \Psi\left(1, \mathbf{X}_{t}\left(M^{c}\right)\right) \\
& +\left(1-\chi\left(\mathbf{1}_{L}, \mathbf{X}_{t}(M)\right)\right) \Psi\left(0, \mathbf{X}_{t}\left(M^{c}\right)\right) \\
& -\left[\chi\left(0_{L}, \mathbf{X}_{t}(M)\right) \Psi\left(1, \mathbf{X}_{t}\left(M^{c}\right)\right)\right. \\
& \left.+\left(1-\chi\left(0_{L}, \mathbf{X}_{t}(M)\right)\right) \Psi\left(0, \mathbf{X}_{t}\left(M^{c}\right)\right)\right] .
\end{aligned}
$$

Portanto

$$
\Phi\left(\mathbf{1}_{L}, \mathbf{X}_{t}\right)-\Phi\left(\mathbf{0}_{L}, \mathbf{X}_{t}\right)=1
$$

se e somente se

$$
\Psi\left(1, \mathrm{X}_{t}\left(M^{c}\right)\right)-\Psi\left(0, \mathrm{X}_{t}\left(M^{c}\right)\right)=1
$$

$e$

$$
\chi\left(1_{L}, \mathbf{X}_{t}(M)\right)-\chi\left(\mathbf{0}_{L}, \mathbf{X}_{t}(M)\right)=1,
$$

o que prova a proposição. 
Corolário 1.27. Sob as hipóteses da Proposição 1.26, temos

$$
I\left(Y_{\Phi}(L)<t \leq T\right)=I\left(Y_{\Psi}(\chi)<t \leq T\right) I\left(Y_{\chi}(L)<t \leq T_{\chi}\right)
$$

onde $T$ é o tempo de vida do sistema $\Phi$ e $T_{\chi}$ é o tempo de vida do módulo $\chi$.

Teorema 1.28. A importância da confiabilidade do módulo $(M, \chi)$ para a confiabilidade do sistema $\Psi$ (ou $\Phi)$ é

$$
I^{\Psi}(\chi)=\sum_{\emptyset \neq L \subset M} I^{\Phi}(L)
$$

\section{Prova:}

Seja $T_{\chi}=\inf \left\{t: \chi\left(\mathbf{X}_{t}(M)\right)=0\right\}$ o tempo de vida do módulo $(M, \chi)$ e seja $A_{\chi}(t)$ o $\mathbf{F}$-compensador do processo $I\left(T_{\chi} \leq t\right)$. Considerando $Y_{\Psi}(\chi)$ o nível crítico do módulo $(M, \chi)$ para o sistema $\Psi$ e $Y_{\chi}(L)$ o nível crítico do bloco $L \subset M$ para o sistema $\chi$. Segue-se da Definição 1.32 que

$$
I^{\Psi}(\chi)=E\left[\int_{0}^{\infty} I\left(Y_{\Psi}(\chi)<t \leq T\right) d A_{\chi}(t)\right] .
$$

Mas pela equação (1.34)

$$
d A_{\chi}(s)=\sum_{\emptyset \neq L \subset M} I\left(Y_{\chi}(L)<s \leq T_{\chi}\right) d A_{L}(s),
$$

e pelo Corolário 1.27 temos,

$$
\begin{aligned}
I^{\Psi}(\chi) & =\sum_{\emptyset \neq L \subset M} E\left[\int_{0}^{\infty} I\left(Y_{\Psi}(\chi)<s \leq T\right) I\left(Y_{\chi}(L)<s \leq T_{\chi}\right) d A_{L}(s)\right] \\
& =\sum_{\emptyset \neq L \subset M} E\left[\int_{0}^{\infty} I\left(Y_{\Phi}(L)<s \leq T\right) d A_{L}(s)\right] \\
& =\sum_{\emptyset \neq L \subset M} I^{\Phi}(L)
\end{aligned}
$$


Corolário 1.29. Se $P\left(S_{i}=S_{j}\right)=0, i \neq j$, então

$$
I^{\Psi}(\chi)=\sum_{\{i\} \subset M} I^{\Phi}(\{i\})
$$




\section{Capítulo 2}

\section{Importância em um Sistema Modelado por uma Cadeia de Markov não homogênea}

\subsection{Introdução}

Neste capítulo estamos interessados em calcular a importância de um componente de um sistema de engenharia $Y_{s}$ modelado por um processo markoviano, com espaço de estados finito de tamanho $N$ admitindo uma representação semimartingal relacionada a seu ambiente, uma família de sub- $\sigma$-álgebras $\left(\Im_{t}\right)_{t \geq 0}$. O sistema depende de um conjunto de componentes que são reparados imediatamente através de um reparo mínimo depois de sua falha, deixando o estado do sistema inalterado.

Assumimos que o processo pontual multivariado dos componentes que compõem o sistema e o próprio sistema não tem falhas simultâneas e as relações entre eles se dão através das suas intensidades de falhas.

Na maioria dos casos de interesse prático, é impossível medir todo o impacto do ambiente no sistema, e assim, observarmos o sistema ao nível de seus componentes. Isto produz uma representação semimartingal que relaciona a dinâmica dos compo- 
nentes à do sistema. A informação (filtragem) dada pela família de sub- $\sigma$-álgebras geradas pelo processo pontual multivariado dos componentes, produz um processo de ganho de inovação correspondente à sua evolução no tempo. Como em Bueno (2000) definimos uma medida de importância do componente $i$ para a confiabilidade de sistema como o valor esperado negativo do ganho de inovação relacionado à primeira falha do componente $i$. Em um caso particular esta medida corresponde a medida de importância de Norros (1986), e generaliza o conceito da importância de Natvig. Resultados de importância de confiabilidade podem ser achadom em Aven (1986), Boland e El-Neweihi (1985), Bueno (2000) e Xie (1988).

Na próxima seção damos uma formulação matemática do problema e recordamos a importância de confiabilidade de um componente $i$ para a confiabilidade do sistema descrita por Bueno (2000). Na Seção 2.3 calculamos a confiabilidade dos componentes e na Seção 2.4 encontramos uma solução explicita para o ganho de inovação no caso $N=2$.

\subsection{Formulação Matemática}

Em várias situações um sistema de engenharia $\left(Y_{t}\right)_{t \geq 0}$, definido em um espaço de probabilidade $(\Omega, \Im, P)$, é determinado por uma representação semimartingal regular (SMR) com relação a seu ambiente $\left(\Im_{t}\right)_{t \geq 0}$, uma família crescente de sub- $\sigma$ álgebras de $\Im$, contínua à direita e completas ( satisfazendo as condições usuais de Dellacherie ), isto é:

$$
Y_{t}=Y_{0}+\int_{0}^{t} f_{s} d s+m_{t}
$$

onde $f_{t}$ é um processo $\Im_{t}$-progressivo, tal que, $E\left[\int_{0}^{t}\left|f_{s}\right| d s\right]<\infty P$-q.c., e $m_{t}$ é um $\Im_{t}$-martingal de média zero. 
Sem perda de generalidade consideramos $\left(\Im_{t}\right)_{t \geq 0}$ definida por

$$
\Im_{t}=\Im_{t}^{Y} \vee \sigma\left\{N_{i}(s), 0 \leq s \leq t, 1 \leq i \leq m\right\}
$$

onde $\Im_{t}^{Y}=\sigma\left\{Y_{s}, 0 \leq s \leq t\right\}$ e $N_{i}(t)=\sum_{j=1}^{\infty} I\left(T_{j} \leq t\right) I\left(V_{j}=i\right)$ é um processo pontual marcado gerado pelos reparos mínimo do componente $i$ com tempos de vidas $T_{j}$ em $\left\{V_{j}=i\right\}$, onde $V_{j}$ é a marca da $j$-ésima falha. Na realidade, $T_{j}$ é o $j$-ésimo tempo de falha dos componentes, $S_{i}, 1 \leq i \leq m$, ordenados de forma crescente. Na maioria dos casos de interesse prático pode-se mostrar a existência da representação anterior diretamente, porém, é impossível observar todo o impacto do ambiente representado pelas $\sigma$-álgebras $\left(\Im_{t}\right)_{t \geq 0}$ sobre o sistema, de forma que nos restringiremos a observar a sub- $\sigma$-álgebras $\left(\Re_{t}\right)_{t \geq 0}$

$$
\Re_{t}=\sigma\left\{N_{i}(s), s \leq t, i=1, \ldots, m\right\} .
$$

Consideremos o processo pontual marcado multivariado $\left(N_{1}(t), \ldots, N_{m}(t)\right)$ gerado pelas falhas dos $m$ componentes que compõem o sistema com $\Re_{t^{-}}$intensidades prevísiveis

$$
\left(\lambda_{1}^{p}(t), \ldots, \lambda_{m}^{p}(t)\right)
$$

e $\Im_{t^{-}}$intensidades

$$
\left(\lambda_{1}(t), \ldots, \lambda_{m}(t)\right)
$$

Projetando o processo $Y_{t}$ sobre $\Re_{t}$, obtemos a função de estrutura $Z_{t}=E\left[Y_{t} \mid \Re_{t}\right]$, e usando a Teoria da Inovação ( veja Apêndice) podemos provar que $Z_{t}$ tem uma representação de semimartingal regular (SMR)(veja Definição (A.3))

$$
Z_{t}=Z_{0}+\int_{0}^{t} g_{s} d s+\sum_{i=1}^{m} \int_{0}^{t} K_{s}(i) d M_{i}^{p}(s)
$$


onde $g_{t}$ é um processo $\Re_{t}$-progressivo tal que $E\left[\int_{0}^{t}\left|g_{s}\right| d s\right]<\infty \quad P$-q.c., $K_{s}(i)$ é um processo $\Re_{s}$-prevísivel, $i=1, \ldots, m$ e $\int_{0}^{t} K_{s}(i) \lambda_{i}^{p}(s) d s<\infty P$-q.c..

No processo descrito em $(2.4), M_{i}^{p}(s)=N_{i}(s)-\int_{0}^{s} \lambda_{i}^{p}(u) d u, i=1, \ldots, m$ é um $\Re_{s}$-martingal, $M^{p}(s)=\left(M_{1}^{p}(s), \ldots, M_{m}^{p}(s)\right)$ é denominado processo de inovação e o processo $K(s)=\left(K_{s}(1), \ldots, K_{s}(m)\right)$ é o ganho de inovação. Se $E\left[f_{t} \mid \Re_{t}\right]$ é $\Re_{t^{-}}$ progressivo podemos usar $g_{t}=E\left[f_{t} \mid \Re_{t}\right]$.

Brémaud (1981) mostra que

$$
K_{t}(i)=\psi_{1, t}(i)-\psi_{2, t}(i)+\psi_{3, t}(i), i=1, \ldots, m
$$

onde $\psi_{1, t}(i), \psi_{2, t}(i)$ e $\psi_{1, t}(i)$ são processos $\Re_{t}$-prevísiveis definidos por

$$
\begin{gathered}
E\left[\int_{0}^{t} C_{s} Y_{s} \lambda_{i}(s) d s\right]=E\left[\int_{0}^{t} C_{s} \psi_{1, s}(i) \lambda_{i}^{p}(s) d s\right] \\
E\left[\int_{0}^{t} C_{s} Y_{s} \lambda_{i}^{p}(s) d s\right]=E\left[\int_{0}^{t} C_{s} \psi_{2, s}(i) \lambda_{i}^{p}(s) d s\right] \\
E\left[\sum_{0 \leq s \leq t} C_{s} \Delta m_{s} \Delta N_{i}(s)\right]=E\left[\int_{0}^{t} C_{s} \psi_{3, s}(i) \lambda_{i}^{p}(s) d s\right]
\end{gathered}
$$

onde $\left(C_{s}\right)_{s \geq 0}$ é $\Re_{s}$-prevísivel.

Usando o fato de que o $\Re_{s}$-martingal $M_{i}^{p}(s)$ em (2.4) é igual a

$$
N_{i}(s)-\int_{0}^{s} \lambda_{i}^{p}(u) d u, i=1, \ldots, m
$$

temos que

$$
Z_{t}=Z_{0}+\int_{0}^{t}\left(g_{s}+\sum_{i=1}^{m} K_{s}(i) \lambda_{i}^{p}(s)\right) d s+\sum_{i=1}^{m} \int_{0}^{t} K_{s}(i) d N_{i}(s)
$$

e portanto os possiveis saltos (pontos de descontínuidades) do processo $Z_{t}$ são refletidos apenas na segunda integral da equação (2.9) e esta por sua vez é diferente de 
zero apenas nos pontos onde ocorrem falhas. Assim o impacto da primeira falha do componente $i$ no processo $Z$ ocorre no intante $S_{i}$ e é dado por:

$$
Z_{S_{i}}-Z_{S_{i}-}=\sum_{j=1}^{m} \int_{S_{i}-}^{S_{i}} K_{s}(j) d N_{j}(s)=K_{S_{i}}(i) .
$$

Por outro lado, na decomposição semimartingal de $Z_{t}$ descrita em (2.4), a parte integral pode ser interpretada como um dispositivo que atualiza as estimativas $E\left[Y_{t} \mid \Re_{t}\right]$ do processo $Y_{t}$ ao longo do tempo. Se o integrando $K_{s}(i)$ não é positivo para todo $i$, então toda falha provoca uma perda no valor esperado. Estes argumentos nos levam à seguinte definição:

Definição 2.1. A importância da confiabilidade do componente $i$ para a confiabilidade do sistema é o valor esperado negativo do ganho de inovação no primeiro tempo de falha $S_{i}$ do componente $i$, isto é,

$$
I(i)=-E\left[K_{S_{i}}(i)\right]=E\left\{E\left[Y_{S_{i}-} \mid \Re_{S_{i}}\right]-E\left[Y_{S_{i}} \mid \Re_{S_{i}}\right]\right\} .
$$

Como um caso particular, podemos obter a versão da importância de Natvig, obtida por Norros (1986b), quando $Y_{t}=E\left[T \mid \Im_{t}\right]$ e $T$ é o tempo de vida do sistema.

Teorema 2.2. Se $T$ é o tempo de vida de um sistema coerente e $Y_{t}=E\left[T \mid \Im_{t}\right]$, onde $\Im_{t}$ é definida como em (2.2) então a importância da confiabilidade do componente $i$ para a confiabilidade do sistema é dado por

$$
I(i)=-E\left[K_{S_{i}}(i)\right]=\operatorname{COV}\left(Y_{S_{i}}, \int_{0}^{S_{i}} \lambda_{i}^{p}(s) d s\right) .
$$


Prova: Aplicando a formula de integração de Dellacherie, temos

$$
\begin{aligned}
E\left[Z_{S_{i}-}\right] & =E\left[\int_{0}^{\infty} Z_{t-} d N_{i}(s)\right] \\
& =E\left[\int_{0}^{\infty} Z_{t-} d \Lambda_{i}^{p}(s)\right] \\
& =E\left[\int_{0}^{\infty} Z_{t} d \Lambda_{i}^{p}(s)\right] \\
& =E\left[Z_{S_{i}} \Lambda_{i}^{p}\left(S_{i}\right)\right] \\
& =E\left[E\left[Y_{S_{i}} \mid \Re_{S_{i}}\right] \Lambda_{i}^{p}\left(S_{i}\right)\right] \\
& =E\left[E\left[\Lambda_{i}^{p}\left(S_{i}\right) Y_{S_{i}} \mid \Re_{S_{i}}\right]\right] \\
& =E\left[\Lambda_{i}^{p}\left(S_{i}\right) Y_{S_{i}}\right] .
\end{aligned}
$$

Além disso,

$$
E\left[Z_{S_{i}}\right]=E\left[\int_{0}^{\infty} Z_{t} d N_{i}(t)\right]=E\left[E\left[Y_{S_{i}} \mid \Re_{S_{i}}\right]\right]=E\left[Y_{S_{i}}\right]
$$

Como os compensadores $\Lambda_{i}^{p}(t)$ nos seus pontos finais $S_{i}, \Lambda_{i}^{p}\left(S_{i}\right), i=1, \ldots, m$ são variáveis aleatórias independentes e com distribuição exponencial de média 1, temos

$$
\begin{aligned}
E\left[K_{S_{i}}(i)\right] & =E\left[Z_{S_{i}}\right]-E\left[Z_{S_{i}-}\right] \\
& =E\left[Y_{S_{i}}\right] E\left[\Lambda_{i}^{p}\left(S_{i}\right)\right]-E\left[\Lambda_{i}^{p}\left(S_{i}\right) Y_{S_{i}}\right] \\
& =-\operatorname{COV}\left(Y_{S_{i}}, \int_{0}^{S_{i}} \lambda_{i}^{p}(s) d s\right)
\end{aligned}
$$

e assim o teorema esta provado. 


\subsection{Importância do Componente}

Consideramos um sistema em multiestados (veja Apêndice), modelado por uma cadeia de Markov não homogênea $\left(X_{t}\right)_{t \geq 0}$, contínua à direita, com valores em $S=$ $\{1, \ldots, N\}$ e parâmetros infinitesimais $q_{i}(t), q_{i j}(t), t \geq 0$, limitados, onde

$$
\begin{gathered}
q_{i}(t)=\lim _{h \rightarrow 0+} \frac{1}{h} P\left(X_{t+h} \neq i \mid X_{t}=i\right), \\
q_{i j}(t)=\lim _{h \rightarrow 0+} \frac{1}{h} P\left(X_{t+h}=j \mid X_{t}=i\right), i, j \in S, i \neq j, \\
q_{i i}(t)=-q_{i}(t)=-\sum_{j \neq i} q_{i j}(t), t \geq 0 .
\end{gathered}
$$

Podemos interpretar o estado 1 como o estado de funcionamento perfeito e o estado $N$ caracterizando condições péssimas de funcionamento. Os outros estados são os estágios intermediários de funcionamento. O sistema $X_{t}$ depende de $m$ componentes com tempos de vida $S_{i}, i=1, \ldots, m$, denotado por $T_{i}, i=1,2, \ldots$, quando as falhas são ordenadas.

Sejam $N_{i}(t)=\sum_{j=1}^{\infty} I\left(T_{j} \leq t\right) I\left(V_{j}=i\right)$ um processo pontual marcado gerado pelos reparos mínimos dos componentes $i, i=1, \ldots, m$ com tempos de vida $T_{j}$ em $\left\{V_{j}=i\right\}$, onde $V_{j}$ é a $j$-ésima marca e $\left(N_{i}(t), i=1, \ldots, m\right)$ é o processo pontual marcado multivariado.

Ambos os processos $\left(N_{i}(t), i=1, \ldots, m\right)$ e $X_{t}$ são definidos em um mesmo espaço de probabilidade $(\Omega, \Im, P)$, são adaptados à $\sigma$-álgebra

$$
\Im_{t}=\Im_{t}^{X} \vee \sigma\left\{N_{i}(s), 0 \leq s \leq t, 1 \leq i \leq m\right\},
$$

onde $\Im_{t}^{X}=\sigma\left\{X_{s}, 0 \leq s \leq t\right\}$ e não tem saltos simultâneos. 
Na dinâmica do tempo a mudança do estado do sistema influencia os estados de seus componentes através de suas intensidades, isto é, as $\Im_{t}$-intensidades dos processos $N_{i}(t), i=1, \ldots, m$,

$$
\lambda_{i}(t)=\mu_{X_{t}}(i, t), i=1, \ldots, m
$$

são dirigidas através de $\left(X_{t}\right)_{t \geq 0}$.

No que segue calcularemos a importância de confiabilidade do componente $i$ para a confiabilidade do sistema ao nível $j$.

Como consequência imediata do Lema $A .26$ temos que o processo

$$
Y_{t}(j)=I\left(X_{t}=j\right)
$$

tem uma representação $\Im_{t}$-semimartingal regular:

$$
Y_{t}(j)=I\left(X_{t}=j\right)=I\left(X_{0}=j\right)+\int_{0}^{t} \sum_{i=1}^{N} I\left(X_{t}=i\right) q_{i j}(s) d s+m_{t}(j)
$$

onde $m_{t}(j)$ é um $\Im_{t}$-martingal, contínuo à direita com limites à esquerda e $m_{0}(j)=$ $0, j=1, \ldots, N$. O nosso interesse é encontrar um estimador para o processo $\left(Y_{t}(j), j \in\right.$ $S)$ e obter os processos de ganhos de inovação $K_{t}(j, i), i=1, \ldots, m, j \in S$, em situações onde o processo $X_{t}$ não é observado e as únicas informações são com respeito à $\sigma$-álgebra

$$
\Re_{t}=\sigma\left\{N_{i}(s), 0 \leq s \leq t, i=1, \ldots, m\right\} .
$$

Um vez que $\left|Y_{t}^{2}(j)\right| \leq 1, j \in S, t \geq 0$, pelo criterio quadrático, (veja o Apêndice) temos que o melhor estimador para o processo $\left(Y_{t}(j), j \in S\right)$ em $\Re_{t}$ é dado pela sua projeção neste espaço, ou seja,

$$
Z_{t}(j)=E\left[Y_{t}(j) \mid \Re_{t}\right], j \in S
$$


Teorema 2.3. O processo $Z_{t}=\left(Z_{t}(1), \ldots, Z_{t}(N)\right)$ é dado por

$$
\begin{aligned}
Z_{t}(j) & =P\left(Y_{0}=j\right)+\sum_{i=1}^{N} \int_{0}^{t} Z_{s}(i) q_{i j}(s) d s \\
& +\sum_{k=1}^{m} \int_{0}^{t} K_{s}(j, k)\left(d N_{k}(s)-\sum_{l \in S} \mu_{l}(k, s) Z_{s^{-}}(l) d s\right)
\end{aligned}
$$

onde

$$
K_{t}(j, i)=-Z_{t^{-}}(j)+\frac{\mu_{j}(i, t) Z_{t^{-}}(j)}{\sum_{l \in S} \mu_{l}(i, t) Z_{t^{-}}(l)},
$$

e $Z_{t^{-}}(j)=\lim _{s \uparrow t} Z_{s}(j), j=1, \ldots, N$

Prova: Por simplicidade provamos para o caso $m=1$, para $m>1$ a prova é similar. Usando a decomposição (2.13) do processo $Y_{t}(j)$ e aplicando o teorema da projeção (veja o Teorema A.12) com respeito a filtragem $\Re_{t}$ definida em (2.14) temos a seguinte representação para o processo $Z_{t}(j)=E\left[Y_{t}(j) \mid \Re_{t}\right]$,

$$
\begin{aligned}
Z_{t}(j) & =P\left(X_{0}=j\right)+\sum_{i=1}^{N} \int_{0}^{t} Z_{s}(i) q_{i j}(s) d s \\
& +\int_{0}^{t} K_{s}(j)\left(d N(s)-\sum_{l \in S} \mu_{l}(s) Z_{s}(l) d s\right)
\end{aligned}
$$

onde $K_{t}(j)$ é o ganho de inovação a ser calculado e $E\left[\mu_{X_{s}}(s) \mid \Re_{s}\right]=\sum_{l \in S} \mu_{l}(s) Z_{s}(l)$ é a $\Re_{s}$-intensidade de $N(s)$, para $l=1, \ldots, N$ uma vez que $\mu_{l}(s), l=1, \ldots, N$ são determinísticos.

O processo $\left(K_{s}\right)_{s \geq 0}$ é dado pela fórmula $K=\psi_{1}-\psi_{2}+\psi_{3}$, veja (2.5), onde os processos $\psi_{j}, j=1,2,3$ são $\Re_{t}$-previsiveis, obtidos através das equações (2.6), (2.7) e (2.8) respectivamente. Como $Y_{t}(j)$ e $N(t)=\sum_{k=1}^{m} N_{k}(t)$ não tem saltos em comum 
temos $\psi_{3}=0$. É imediato que $\psi_{2, t}(j)=Z_{t^{-}}(j)$. O processo $\psi_{1, t}(j)$ é obtido por:

$$
E\left[\int_{0}^{t} C_{s} Y_{s}(j) \mu_{X_{s}}(s) d s\right]=E\left[\int_{0}^{t} C_{s} \psi_{1, s}(j) \sum_{l \in S} \mu_{s}(l) Z_{s}(l) d s\right] .
$$

Como $\mu_{X_{s}}(s) Y_{s}(j)=\mu_{j}(s) Y_{s}(j)$ e $Z_{s}(l) d s=Z_{s^{-}}(l) d s, l \in S$, temos

$$
E\left[\int_{0}^{t} C_{s} Y_{s}(j) \mu_{X_{s}}(s) d s\right]=E\left[\int_{0}^{t} C_{s} Y_{s}(j) \mu_{j}(s) d s\right] .
$$

Usando (2.18) e (2.19), concluimos que

$$
\psi_{1, t}=\frac{\mu_{j}(t) Z_{t^{-}}(j)}{\sum_{l \in S} \mu_{l}(t) Z_{t^{-}}(l)}
$$

Portanto

$$
K_{t}(j)=-Z_{t^{-}}(j)+\frac{\mu_{j}(t) Z_{t^{-}}(j)}{\sum_{l \in S} \mu_{l}(t) Z_{t^{-}}(l)}
$$

e o teorema está provado.

Reformulando a Definição (2.1) para este caso particular obtemos:

Definição 2.4. A importância da confiabilidade do componente $i$ para a confiabilidade do sistema no nível $j, j \in S$, é o valor esperado negativo do processo do ganho de inovação correspondente ao primeiro instante de falha $S_{i}$ para o estado $j$ :

$$
I(i, j)=-E\left[K_{S_{i}}(j, i)\right]=E\left[Z_{S_{i}^{-}}(j)\right]-E\left[\frac{\mu_{j}\left(i, S_{i}\right) Z_{S_{i}^{-}}(j)}{\sum_{l \in S} \mu_{l}\left(i, S_{i}\right) Z_{S_{i}^{-}}(l)}\right] .
$$


Observações 2.5. i) Pelo Teorema 2.3 podemos escrever:

$$
\begin{aligned}
& Z_{t}(j)=P\left(Y_{0}=j\right)+\sum_{l \in S} \int_{0}^{t} Z_{s}(l) q_{l j}(s) d s \\
& +\sum_{k=1}^{m} \int_{0}^{t}\left[-Z_{s^{-}}(j)+\frac{\mu_{j}(k, s) Z_{s^{-}}(j)}{\sum_{l \in S} \mu_{l}(k, s) Z_{s^{-}}(l)}\right] d N_{k}(s) \\
& -\sum_{k=1}^{m} \int_{0}^{t}\left[-Z_{s^{-}}(j)+\frac{\mu_{j}(k, s) Z_{s^{-}}(j)}{\sum_{l \in S} \mu_{l}(k, s) Z_{s^{-}}(l)}\right] \sum_{l \in S} \mu_{l}(k, s) Z_{s^{-}}(l) d s, \\
& =P\left(Y_{0}=j\right)+\underbrace{\sum_{l \in S} \int_{0}^{t} Z_{s}(l) q_{l j}(s) d s}_{I}+\underbrace{\sum_{k=1}^{m} \int_{0}^{t} Z_{s^{-}}(j) \sum_{l \in S} \mu_{l}(k, s) Z_{s^{-}}(l) d s}_{I I}, \\
& -\underbrace{\sum_{k=1}^{m} \int_{0}^{t} \mu_{j}(k, s) Z_{s^{-}}(j) d s}_{I I I} \\
& +\sum_{k=1}^{m}\left[\sum_{n \geq 0}\left(-Z_{T_{n}-}(j)+\frac{\mu_{j}\left(k, T_{n}\right) Z_{T_{n}-}(j)}{\sum_{l \in S} \mu_{l}\left(k, T_{n}\right) Z_{T_{n}}(l)}\right) I\left(T_{n} \leq t, V_{n}=k\right)\right] . \\
& \text { Como } I+I I-I I I=\int_{0}^{t} \sum_{l \in S} Z_{s}(l)\left[q_{l j}(s)+Z_{s}(j)\left(\sum_{k=1}^{m}\left(\mu_{l}(k, s)-\mu_{j}(k, s)\right)\right)\right] d s, \text { temos } \\
& Z_{t}(j)=P\left(Y_{0}=j\right)+\int_{0}^{t} \sum_{l \in S} Z_{s}(l)\left[q_{l j}(s)+Z_{s}(j)\left(\sum_{k=1}^{m}\left(\mu_{l}(k, s)-\mu_{j}(k, s)\right)\right)\right] d s \\
& +\sum_{k=1}^{m}\left[\sum_{n \geq 0}\left(-Z_{T_{n}}-(j)+\frac{\mu_{j}\left(k, T_{n}\right) Z_{T_{n}}(j)}{\sum_{l \in S} \mu_{l}\left(k, T_{n}\right) Z_{T_{n}}-(l)}\right) I\left(T_{n} \leq t, V_{n}=k\right)\right] \cdot(2.20)
\end{aligned}
$$

Equivalentemente, se $t$ está entre os saltos do processo $N(t)=\sum_{k=1}^{m} N_{k}(t)$, isto é, $T_{n} \leq t<T_{n+1}$, temos

$$
\begin{aligned}
Z_{t}(j)=Z_{T_{n}}(j)+ & \int_{T_{n}}^{t} \sum_{l \in S} Z_{s}(l)\left[q_{l j}(s)\right. \\
& \left.+Z_{s}(j)\left(\sum_{k=1}^{m}\left(\mu_{l}(k, s)-\mu_{j}(k, s)\right)\right)\right] d s
\end{aligned}
$$


Nos saltos

$$
Z_{T_{n}}(j)=\sum_{k=1}^{m}\left[\frac{\mu_{j}\left(k, T_{n}\right) Z_{T_{n}^{-}}(j)}{\sum_{l \in S} \mu_{l}\left(k, T_{n}\right) Z_{T_{n}^{-}}(l)} I\left(V_{n}=k\right)\right], j \in S .
$$

ii) Entre os saltos $T_{n}<t<T_{n+1}, n \in\{0,1, \ldots\},\left(T_{0}=0\right)$, vale:

$$
\frac{d Z_{t}(j)}{d t}=\sum_{l \in S} Z_{t}(l)\left[q_{l j}(t)+Z_{t}(j)\left(\sum_{k=1}^{m}\left(\mu_{l}(k, t)-\mu_{j}(k, t)\right)\right)\right] .
$$

\subsection{Aplicação: O Caso para $N=2$ Estados}

Quando $N=2$ podemos obter uma solução explicita para equação diferencial (2.23). Se assumirmos que $P\left(X_{0}=1\right)=1$, então para todo $t$ tal que $T_{n}<t<T_{n+1}$ temos:

$$
\frac{d Z_{t}(1)}{d t}=\sum_{l=1}^{2} Z_{t}(l)\left[q_{l 1}(t)+Z_{t}(1)\left(\sum_{k=1}^{m}\left(\mu_{l}(k, t)-\mu_{1}(k, t)\right)\right)\right] .
$$

Como $Z_{s}(2)=1-Z_{s}(1)$

$$
\begin{aligned}
\frac{d Z_{t}(1)}{d t} & =-Z_{t}(1)\left[Z_{t}(1)\left(\sum_{k=1}^{m}\left(\mu_{2}(k, t)-\mu_{1}(k, t)\right)\right)\right. \\
& \left.+q_{12}(t)+q_{21}(t)-\sum_{k=1}^{m}\left(\mu_{2}(k, t)-\mu_{1}(k, t)\right)\right]+q_{21}(t) .
\end{aligned}
$$

O processo $Z_{t}(1)$ pode ser deteminado explicitamente da equação diferencial (2.25), considerando com o valor inicial $Z_{0}(1)=1$. Procedemos com a seguinte aplicação:

Teorema 2.6. Seja

$$
q_{1}(t)=q_{12}(t)>0, q_{21}(t)=0 e \frac{q_{12}(t)}{\sum_{k=1}^{m}\left(\mu_{2}(k, t)-\mu_{1}(k, t)\right)}=c,
$$

onde c é uma constante. Então a equação (2.25) tem a seguinte solução:

Entre os saltos, $T_{n} \leq t<T_{n+1}, n \in\{0,1, \ldots\}$. 
1) Se $c \neq 1$ temos

$$
Z_{t}(1)=\frac{(c-1) Z_{T_{n}}(1) h(t)}{Z_{T_{n}}(1)(1-h(t))+c-1}
$$

onde,

$$
h(t)=\exp \left\{-(c-1) \int_{T_{n}}^{t} \sum_{k=1}^{m}\left(\mu_{2}(k, s)-\mu_{1}(k, s)\right) d s\right\} .
$$

2) $\operatorname{Sec}=1$ temos

$$
Z_{t}(1)=\frac{Z_{T_{n}}(1)}{1+Z_{T_{n}}(1) \sum_{k=1}^{m} \int_{T_{n}}^{t}\left(\mu_{2}(k, s)-\mu_{1}(k, s)\right) d s} .
$$

Se $t=T_{n}$ para algum $n \in\{0,1, \ldots\}$, temos

$$
Z_{T_{n}}(1)=\sum_{k=1}^{m}\left[\frac{\mu_{1}\left(k, T_{n}\right) Z_{T_{n}}(1)}{\left(\mu_{1}\left(k, T_{n}\right)-\mu_{2}\left(k, T_{n}\right)\right) Z_{T_{n}}(1)+\mu_{2}\left(k, T_{n}\right)} I\left(V_{n}=k\right)\right],
$$

onde $Z_{T_{0}}(1)=1$.

Prova: Considerando a condição (2.26), podemos reescrever (2.25) da seguinte forma:

Se $c \neq 1$,

$$
\frac{d Z_{t}(1)}{Z_{t}(1)\left[Z_{t}(1)+c-1\right]}=-\sum_{k=1}^{m}\left(\mu_{2}(k, t)-\mu_{1}(k, t)\right) d t
$$

se $c=1$,

$$
\frac{d Z_{t}(1)}{Z_{t}^{2}(1)}=-\sum_{k=1}^{m}\left(\mu_{2}(k, t)-\mu_{1}(k, t)\right) d t .
$$

Portanto $Z_{t}(1)$ pode ser obtido explicitamente como a solução das equações diferenciais acima dado o valor inicial $Z_{0}(1)=1$ e o resultado esta provado. 
Corolário 2.7. Se $q_{12}(t)=q>0, \mu_{1}(k, t)=\mu_{1}(k)$, e $\mu_{2}(k, t)=\mu_{2}(k), k=1, \ldots, m$, são constantes, então:

$$
\begin{aligned}
\text { Para } q \neq \sum_{k=1}^{m}\left(\mu_{2}(k)-\mu_{1}(k)\right) & =\bar{c} \text { temos } \\
Z_{t}(1) & =\frac{(q-\bar{c}) Z_{T_{n}}(1) h(t)}{\bar{c} Z_{T_{n}}(1)(1-h(t))+q-\bar{c}}
\end{aligned}
$$

onde,

$$
h(t)=\exp \left\{(\bar{c}-q)\left(t-T_{n}\right)\right\}
$$

para $T_{n} \leq t<T_{n+1}, n \in\{0,1, \ldots\}$.

Para $q=\sum_{k=1}^{m}\left(\mu_{2}(k)-\mu_{1}(k)\right)=\bar{c}$ temos

$$
Z_{t}(1)=\frac{Z_{T_{n}}(1)}{1+\widehat{Z}_{T_{n}}(1) \bar{c}\left(t-T_{n}\right)},
$$

para todo $t \geq 0$, tal que, $T_{n} \leq t<T_{n+1}, n \in\{0,1, \ldots\}$.

Observações 2.8. i) O processo $Z_{t}(2)$ é determinado explicitamente pelo fato de que $Z_{t}(2)=1-Z_{t}(1)$.

ii)Se $q_{12}(t)=q, \mu_{1}(k, t)=\mu_{1}(k)$, and $\mu_{2}(k, t)=\mu_{2}(k)$ são constantes, então $o$ caso $N=2$ é semelhante a um problema bem conhecido como o problema de detecção do tempo de desordem:

Em uma tempo aleatório não-observável $T$ existem ocorrências de trocas de características de algum fenômeno aleatório observável (como, por exemplo, uma troca de intensidades). O problema é detectar, "se possivel,"este tempo de desordem através das médias condicionais dos processos aleatórios observados (veja Brémaud (1981)).

iii) Sob as condições do Corolário 2.7, para $0<q<\bar{c}$, temos que $Z_{t}(2)$ é crescente ( portanto $Z_{t}(1)$ é decrescente) até o nivel $Z_{t}(2)<\frac{q}{\bar{c}}=r$. Quando $Z_{t}(2)$ salta 
acima deste nível, então $Z_{t}(2)$ decresce $\left(Z_{t}(1)\right.$ cresce) entre os saltos mas não abaixo do nivel $r$.

iv) Quando $\mu_{1}(k)=\mu_{2}(k), k=1, \ldots, m$, são constantes, ou seja, as taxas de falhas não dependem do estado do processo $X_{t}$, os saltos de $Z_{t}(2)$ são nulos, e além disso, $Z_{t}(2)=P\left(T \leq t \mid N_{i}(s), s \leq t, i=1, \ldots, m\right)=1-\exp (-q t)$ é completamente deterministico.

Exemplo 2.9. (Estimatição Recursiva do Ganho de Innovação.)

Seja

$$
\begin{gathered}
N=2, m=2, q_{1}(t)=q_{12}(t)=\frac{1}{2}, q_{21}(t)=0, \mu_{1}(1, t)=1, \\
\mu_{2}(1, t)=2, \mu_{1}(2, t)=2, \mu_{2}(2, t)=3, t \geq 0, \text { e } P\left(Y_{0}=1\right)=1 .
\end{gathered}
$$

Pelo Corolario 2.7 temos:

$\operatorname{Para} T_{n}<t<T_{n+1}$

$$
\begin{aligned}
& K_{t}(1, k)=-Z_{t}(1)+\frac{\mu_{1}(k, t) Z_{t}(1)}{\mu_{2}(k, t)-Z_{t}(1)\left(\mu_{2}(k, t)-\mu_{1}(k, t)\right.}, k=1,2, \\
& K_{t}(2, k)=-Z_{t}(2)+\frac{\mu_{2}(k, t) Z_{t}(2)}{Z_{t}(2)\left(\mu_{2}(k, t)-\mu_{1}(k, t)\right)+\mu_{1}(k, t)}, k=1,2,
\end{aligned}
$$

onde,

$$
\begin{gathered}
Z_{t}(1)=\frac{3 Z_{T_{n}}(1) \exp \left(\frac{3}{2}\left(t-T_{n}\right)\right)}{4 Z_{T_{n}}(1)\left(\exp \left(\frac{3}{2}\left(t-T_{n}\right)\right)-1\right)+3}, \\
Z_{T_{n}}(1)=\frac{Z_{T_{n}^{-}}(1)}{2-Z_{T_{n}^{-}}(1)} I\left(V_{n}=1\right)+\frac{2 Z_{T_{n}^{-}}(1)}{3-Z_{T_{n}^{-}}(1)} I\left(V_{n}=2\right)
\end{gathered}
$$




$$
Z_{T_{n}^{-}}(1)=\frac{3 Z_{T_{n-1}}(1) \exp \left(\frac{3}{2}\left(T_{n}-T_{n-1}\right)\right)}{4 Z_{T_{n-1}}(1)\left(\exp \left(\frac{3}{2}\left(T_{n}-T_{n-1}\right)\right)-1\right)+3}
$$

e $Z_{t}(2)=1-Z_{t}(1), Z_{T_{0}}(1)=1$

Para $t=T_{n}, n \in\{0,1, \ldots\}$ onde $T_{0}=0$, temos

$$
\begin{aligned}
K_{T_{n}}(j, k) & =-Z_{T_{n}^{-}}(j)+\frac{\mu_{j}\left(k, T_{n}\right) Z_{T_{n}^{-}}(j)}{\mu_{2}\left(k, T_{n}\right)-Z_{T_{n}^{-}}(1)\left(\mu_{2}\left(k, T_{n}\right)-\mu_{1}\left(k, T_{n}\right)\right)} \\
& =-Z_{T_{n}^{-}}(j)+\frac{\mu_{j}\left(k, T_{n}\right) Z_{T_{n}^{-}}(j)}{\mu_{2}\left(k, T_{n}\right)-Z_{T_{n}^{-}}(1)}
\end{aligned}
$$

$k=1,2$, e $j=1,2$.

Exemplo 2.10. Diferentes valores para $q, \mu_{1}(k), \mu_{2}(k), k=1,2$ são escolhidos. Para cada conjunto de parâmetros 100 simulações foram realizadas gerando amostras do processo $(N(1), N(2))$ onde cada realização resultou em uma realização de $Z .(j), S_{k}$, e $K_{S_{k}}(j, k), k=1,2, j=1,2$ onde $S_{k}$ é o tempo da primeira falha do componente $k$.

A Tabela 2.1 mostra, para cada conjunto de parâmetros $q, \mu_{1}(k), \mu_{2}(k), k=1,2$, as médias amostrais denotadas por $K(j, k), j=1,2, k=1,2$ das 100 realizações de $K_{S_{k}}(j, k)$, e suas respectivas variâncias amostrais denotadas por $V K(j, k)$.

$-K(j, k)$ é um estimador não-viesado para $-E\left[K_{S_{k}}(j, k)\right]=I(k, j)$ onde $I(k, j)$ é a importância da confiabilidade do componente $k$ para a confiabilidade do sistema no nível $j$. 


\begin{tabular}{|r|l|l|l|l|}
\hline $\mathrm{q}$ & 0.5 & 0.5 & 2 & 10 \\
\hline$\mu_{1}(1)$ & 1 & 0.25 & 0.25 & 3 \\
\hline$\mu_{2}(1)$ & 2 & 1 & 0.5 & 5 \\
\hline$\mu_{1}(2)$ & 2 & 2 & 1 & 2 \\
\hline$\mu_{2}(2)$ & 3 & 3 & 1 & 8 \\
\hline$-K(1,1)$ & 0.1196031 & 0.2774918 & 0.03230153 & 0.05897765 \\
\hline$V K(1,1)$ & 0.002638685 & 0.004367538 & 0.002513032 & 0.001971568 \\
\hline$-K(2,1)$ & 0.04978108 & 0.04795007 & 0 & 0.1127323 \\
\hline$V K(2,1)$ & 0.0007942565 & 0.0007094964 & 0 & 0.01380347 \\
\hline$-K(1,2)$ & -0.1196031 & -0.2774918 & -0.03230153 & -0.05897765 \\
\hline$V K(1,2)$ & 0.002638685 & 0.004367538 & 0.002513032 & 0.001971568 \\
\hline$-K(2,2)$ & -0.04978108 & -0.04795007 & 0 & -0.1127323 \\
\hline$V K(2,2)$ & 0.0007942565 & 0.0007094964 & 0 & 0.01380347 \\
\hline
\end{tabular}

Tabela 2.1: 


\section{Capítulo 3}

\section{Um modelo de Burn-in}

\subsection{Introdução}

O Burn-in é um procedimento usado em engenharia para eliminar itens defeituosos em uma linha de produção. Uma linha de produção consiste usualmente na fabricação de componentes que são estruturalmente organizados formando desse modo um sistema. Podemos considerar, por exemplo, sistemas que são circuítos eletrônicos compostos por vários tipos de chips. Os sistemas, assim como os componentes, operam por um período de tempo e depois falham. Geralmente sistemas formados por componentes eletrônicos tem tempos de vidas relativamente longos ou podem falhar precocemente.

Para assegurar que somente os objetos (sistemas ou componentes) "perfeitos"sejam colocados no mercado o fabricante submeterá por um período de tempo especificado todos os componentes ( ou uma amostra representativa destes) a testes que simulam situações típicas do uso do componente no mercado ou até mais severas. Estes tipos de testes também podem ser realizados em sistemas com o objetivo de determinar os que são defeituosos ou os que apresentam defeitos estruturais. Estes testes ( ou procedimentos) tem uma variedade de nomes, dependendo da área de 
aplicação, e usaremos o termo burn-in para descrevê-los. Muitas das idéias tradicionais concernentes ao burn-in são discutidas no livro de Jensen e Peterson (1982).

Quando decidimos por um procedimento de burn-in, devemos considerar alguns critérios básicos segundo os quais passamos a determinar o tempo ótimo de Burnin. Para tanto consideramos uma estrutura de custo, devido às falhas ao longo do tempo, descritas por um processo $\left(Z_{t}\right)_{t \geq 0}$ e uma estrutura de ganho por unidade de tempo, caso não haja falhas, determinada por um processo $\left(X_{t}\right)_{t \geq 0}$. Analisaremos o problema de burn-in segundo dois critérios:

Problema 1: Encontrar o instante de burn-in ( $\Im_{t}$-tempo de parada) ótimo que maximize a diferença entre os processos de ganho e de custo, isto é, $K_{t}^{1}=X_{t}-Z_{t}$.

Problema 2: Encontrar o instante de burn-in ( $\Im_{t}$-tempo de parada) ótimo que minimize a razão entre os processos de custo e de ganho, isto é, $K_{t}^{2}=\frac{Z_{t}}{X_{t}}$.

O Problema 1 foi desenvolvido por Aven e Jensen (1999) e é apresentado neste trabalho com o objetivo de intepretação e exemplificação.

Em nosso contexto e baseado nas informações dos tempos de vida de $m$ objetos colocados em teste usaremos uma representação semimartingal regular (SMR) (veja Definição A.3) para determinar os tempos ótimos de Burn-in.

\subsection{Formulação Matemática}

Consideremos os tempos de vida de $m$ objetos (sistemas ou componentes), $T_{j}, j=$ $1, \ldots, m$, que são submetidos a um procedimento de burn-in. Assumimos que $T_{j}, j=$ $1, \ldots, m$, são variáveis aleatórias finitas, positivas, independentes e estão definidas em um mesmos espaço de probabilidade completo $(\Omega, \Im, P)$. 
Durante o procedimento os objetos são colocados em condições severas de uso (condições de forte estresses) de modo que a taxa de falha durante e depois do burn-in, sejam $\lambda_{j}^{0}(t), \lambda_{j}^{1}(t)$, respectivamente, onde, $\lambda_{j}^{0}(t) \geq \lambda_{j}^{1}(t)$ para todo $t \geq 0$.

A formulação matemática das observações é determinada por um família de sub$\sigma$-álgebras de $\Im, \mathbf{F}=\left(\Im_{t}\right)_{t \geq 0}$ onde

$$
\Im_{t}=\sigma\left\{I\left(T_{j}>s\right), 0 \leq s \leq t, j=1, \ldots, m\right\} .
$$

Vamos assumir que o tempos de vidas $T_{j}, j=1, \ldots, m$, tem médias finitas, isto é, $E T_{i}<\infty, i=1, \ldots, m$ e admitam uma representação $\Im_{t}$-semimartingal regular, isto é

$$
I\left(T_{j} \leq t\right)=\int_{0}^{t} I\left(T_{j}>s\right) \lambda_{j}^{Y_{s}}(s) d s+M_{j}(t), j=1, \ldots, m
$$

onde $Y_{t}=I(\tau<t)$ e $\tau$ é um $\Im_{t}$-tempo de parada que representa o tempo de burn-in, e $M_{j}$ é um $\Im_{t}$-martingal de média zero e limitado em $L^{2}\left(\sup _{t \geq 0} E\left|M_{j}(t)\right|^{2}<\infty\right)$.

Observação 3.1. A representação (3.1) (veja Aven e Jensen (1999)) pode ser obtida da seguinte maneira: Observe que o tempo de vida do j-ésimo objeto pode ser escrito como

$$
T_{j}=L_{j} \wedge \tau+R_{j} I\left(L_{j}>\tau\right)
$$

onde $L_{j}, R_{j}, j=1, \ldots, n$, são variáveis aleatórias independentes; $a \wedge b$ denota $o$ mínimo de a e b; $L_{j}$ é tempo de vida do item $j$ quando é exposto a um nível de estresse e $R_{j}$ é o tempo de operação se o item sobrevive a fase do burn-in $\tau$. Sejam $F_{j}$ a função de distribuição do tempo de vida $T_{j}, H_{j}$ a função de distribuição de $L_{j}, j=1, \ldots, n$, tais que $H_{j}(0)=F_{j}(0)=0, \bar{H}_{j}(t)=1-H_{j}(t), \bar{F}_{j}(t)=1-F_{j}(t)$. Além disso, assumiremos que $H_{j}$ e $F_{j}$ são absolutamente contínuas, com funções 
densidades $h_{j}$ e $f_{j}$, respectivamente e que o tempo de operação $R_{j}$ tem função de sobrevivência condicional correspondente a $F_{j}$ :

$$
P\left(T_{j} \leq t+s \mid \tau=t<L_{j}\right)=P\left(R_{j} \leq s \mid \tau=t<L_{j}\right)=\frac{F_{j}(t+s)-F_{j}(t)}{\bar{F}_{j}(t)}, t, s \in \mathbb{R}_{+}
$$

Os processo de custos e de recompensa são definidos da seguinte maneira:

Consideremos uma recompensa, denotada por $C, C>0$, por unidade de tempo operacional para os objetos que sobrevivem ao tempo de burn-in. O processo de custo é composto dos custos das falhas durante o procedimento de burn-in que denotaremos por $C_{B}, C_{B}>0$ e dos custos das falhas depois do procedimento de burn-in denotados por $C_{F}, C_{F}>C_{B}$.

Fixando o tempo de burn-in, $\tau=t$, podemos definir o processo de custo total por

$$
Z_{t}=C_{B} \sum_{j=1}^{m} I\left(T_{j} \leq t\right)+C_{F} \sum_{j=1}^{m} I\left(T_{j}>t\right)=\left(C_{B}-C_{F}\right) \sum_{j=1}^{m} I\left(T_{j} \leq t\right)+m C_{F}
$$

e através de (3.1) podemos representa-lo como um semimartingal regular (SMR):

$$
Z_{t}=m C_{F}+\left(C_{B}-C_{F}\right) \int_{0}^{t} \sum_{j=1}^{m} I\left(T_{j}>s\right) \lambda_{j}^{0}(s) d s+\sum_{j=1}^{m} M_{j}(t) \text { ? }
$$

O processo recompensa é definido por

$$
\hat{X}_{t}=C \sum_{j=1}^{m}\left(T_{j}-t\right)^{+}
$$

O processo de custo total $Z_{t}$ é $\Im_{t}$-adaptado. Entretanto $\hat{X}_{t}$ não é, isto é, não podemos observar o processo recompensa $\hat{X}_{t}$ em $\Im_{t}$. Consideramos o processo

$$
X_{t}=E\left[\hat{X}_{t} \mid \Im_{t}\right]=C \sum_{j=1}^{m} I\left(T_{j}>t\right) \mu_{j}(t)
$$


onde $\mu_{j}(t)=E\left[\left(T_{j}-t\right)^{+} \mid T_{j}>t\right]=\frac{1}{\bar{F}_{j}(t)} \int_{t}^{\infty} \bar{F}_{j}(x) d x, j=1, \ldots, m$. Agora $\left(X_{t}\right)_{t \geq 0}$ e $\left(Z_{t}\right)_{t \geq 0}$ são $\Im_{t}$-adaptados com trajetórias contínuas à direita.

Assumindo que a média residual $\mu_{j}(t)=\frac{1}{\bar{F}_{j}(t)} \int_{t}^{\infty} \bar{F}_{j}(x) d x, j=1, \ldots, m$, tem derivadas em relação a $t, t \geq 0$, dada por $\mu_{j}^{\prime}(t)=-1+\lambda_{j}^{1}(t) \mu_{j}(t)$, Aven e Jensen (1999) provou o seguinte resultado:

Teorema 3.2. (Aven e Jensen (1999))

O processo $X_{t}=C \sum_{j=1}^{m} I\left(T_{j}>t\right) \mu_{j}(t)$ definido acima, tem uma representação semimartingal regular (SMR) dada por:

$$
X_{t}=X_{0}+C \int_{0}^{t} \sum_{j=1}^{m} I\left(T_{j}>s\right)\left[-1-\mu_{j}(s)\left(\lambda_{j}^{0}(s)-\lambda_{j}^{1}(s)\right)\right] d s+N_{t},
$$

onde $N_{t}$ é um martingal limitado em $L^{2}$, e $X_{0}=C \sum_{j=1}^{m} \mu_{j}(0)$.

Prova: Para obter uma representação semimartingal de $X_{t}$, é suficiente conseguir uma representação para o processo $I\left(T_{j}>t\right) \mu_{j}(t)$. Como $\mu_{j}($.$) e I\left(T_{j}>\right.$.) são contínuas à direita e de variação limitada em $[0, t]$, podemos usar a fórmula de integração por partes de Stieltjes obtendo

$$
\begin{aligned}
I\left(T_{j}>t\right) \mu_{j}(t) & =I\left(T_{j}>0\right) \mu_{j}(0)+\int_{0}^{t} \mu_{j}\left(s^{-}\right) d I\left(T_{j}>s\right) \\
& +\int_{0}^{t} I\left(T_{j}>s\right) d \mu_{j}(s) .
\end{aligned}
$$

Substituindo

$$
I\left(T_{j}>s\right)=1+\int_{0}^{s}\left(-I\left(T_{j}>x\right) \lambda_{j}^{0}(x)\right) d x+M_{j}(s)
$$


(veja (3.1)) nesta fórmula e usando a continuidade de $\mu$ temos

$$
\begin{aligned}
I\left(T_{j}>t\right) \mu_{j}(t) & =\mu_{j}(0)+\int_{0}^{t}\left[-\mu_{j}(s) I\left(T_{j}>s\right) \lambda_{j}^{0}(s)+I\left(T_{j}>s\right) \mu_{j}^{\prime}(s)\right] d s \\
& +\int_{0}^{t} \mu_{j}(s) d M_{j}(s) \\
& =\mu_{j}(0)+\int_{0}^{t} I\left(T_{j}>s\right)\left[-1-\mu_{j}(s)\left(\lambda_{j}^{0}(s)-\lambda_{j}^{1}(s)\right)\right] d s \\
& +\widetilde{M}_{j}(t)
\end{aligned}
$$

onde $\widetilde{M}_{j}(t)=\int_{0}^{t} \mu_{j}(s) d M_{j}(s)$ é um martingal de média zero, com limite em $L^{2}$. Isto nos garante a seguinte representação semimartingal para $X$ :

$$
X_{t}=C \sum_{j=1}^{m} \mu_{j}(0)+C \int_{0}^{t} \sum_{j=1}^{m} I\left(T_{j}>s\right)\left[-1-\mu_{j}(s)\left(\lambda_{j}^{0}(s)-\lambda_{j}^{1}(s)\right)\right] d s+N_{t},
$$

onde $N_{t}=C \sum_{j=1}^{m} \widetilde{M}_{j}(t)$, é um martingal de média zero e limitado em $L^{2}$ (portanto uniformemente integrável).

Definindo

$$
C_{T}^{\mathrm{F}}=\left\{\tau: \tau \text { é um } \Im_{t}-\text { tempo de parada }, \tau \leq T\right\},
$$

onde $T=\max \left\{T_{1}, T_{2}, \ldots, T_{m}\right\}$ como a coleção dos $\Im_{t}$-tempos de paradas admissiveis, consideramos dois problemas:

Problema 1. Seja o processo $K_{t}^{1}=X_{t}-Z_{t}$. O problema de burn-in é encontrar um tempo de parada ótimo $\sigma \in C_{T}^{\mathrm{F}}$ tal que:

$$
K^{*}=E K_{\sigma}^{1}=\sup \left\{E K_{\tau}^{1}: \tau \in C_{T}^{\mathrm{F}}\right\} \text {. }
$$

Problema 2. Seja o processo $K_{t}^{2}=\frac{Z_{t}}{X_{t}}$. O problema de burn-in é encontrar um tempo de parada ótimo $\sigma \in C_{T}^{\mathrm{F}}$ tal que:

$$
K_{*}=E K_{\sigma}^{2}=\inf \left\{E K_{\tau}^{2}: \tau \in C_{T}^{\mathrm{F}}\right\} .
$$


Na busca de uma solução para os problemas acima, vamos usar uma condição denominada caso monótono e uma regra de parada chamada de IOPF (infinitesimalolhada-para-frente), cujas definições são dadas por:

Definição 3.3. (MON) Seja $Z$ um semimartingal regular (SMR), com a seguinte representação:

$$
Z_{t}=Z_{0}+\int_{0}^{t} f_{s} d s+M_{t}
$$

Então a seguinte condição

$$
\left\{f_{t} \leq 0\right\} \subseteq\left\{f_{t+h} \leq 0\right\}, \forall t, h \in \mathbb{R}_{+}, \bigcup_{t \in \mathbb{R}_{+}}\left\{f_{t} \leq 0\right\}=\Omega
$$

é chamada de caso monótono. O tempo de parada

$$
\left.\sigma=\inf \left\{t \in \mathbb{R}_{+}: f_{t} \leq 0\right\}\right\}
$$

é chamada regra de parada IOPF (infinitesimal-olhada-para-frente).

Note que a regra de parada IOPF denotada por $\sigma$ é o candidato natural para resolver problemas de maximização, onde a condição (MON) esteja satisfeita. Jensen (1989) prova o seguinte teorema:

Teorema 3.4. (Jensen (1989))

Seja $Z$ um semimartingal regular (SMR) com a representação (3.4) e seja $\sigma$ a regra de parada IOPT. Se a condição (3.5) esta satisfeita e a parte martingal $M$ é u.i., então

$$
E Z_{\sigma}=\sup \left\{E Z_{\tau}: \tau \text { tempo de parada }\right\}
$$




\subsection{Soluções para os Problemas}

\subsubsection{Uma Solução para o Problema 1}

Da condição (3.1) e pelo Teorema 3.2 temos que o processo $K_{t}^{1}=X_{t}-Z_{t}$ tem uma representação semimartingal regular (SMR),

$$
K_{t}^{1}=X_{0}-Z_{0}+\int_{0}^{t} \sum_{j=1}^{m} I\left(T_{j}>s\right) g_{j}(s) d s+L_{t},
$$

onde

$$
\begin{gathered}
g_{j}(s)=C\left(-1-\mu_{j}(s)\left(\lambda_{j}^{0}(s)-\lambda_{j}^{1}(s)\right)\right)+\left(C_{F}-C_{B}\right) \lambda_{j}^{0}(s) \\
X_{0}=C \sum_{j=1}^{m} \mu_{j}(0), Z_{0}=m C_{F} \text { e } L_{t}=C \sum_{j=1}^{m} \widetilde{M}_{j}(t)+\left(C_{F}-C_{B}\right) \sum_{j=1}^{m} M_{j}(t) \text { com }
\end{gathered}
$$
$\widetilde{M}_{j}(t), M_{j}(t)$ martingais u.i. de média zero. Assim $L=\left(L_{t}\right)_{t \geq 0}$ é um martingal u.i. de média zero.

Teorema 3.5. Suponhamos que as funções $g_{j}, j=1, \ldots, m$ definidas em (3.7) satisfaçam a seguinte condição

$$
\sum_{j \in \gamma} g_{j}(t) \leq 0 \Rightarrow g_{j}(s) \leq 0, \forall j \in \gamma \subseteq\{1, \ldots, m\}, \forall s \geq t
$$

então as condições

$$
\begin{gathered}
A_{t}=\left\{\sum_{j=1}^{m} I\left(T_{j}>t\right) g_{j}(t) \leq 0\right\} \subseteq\left\{\sum_{j=1}^{m} I\left(T_{j}>t+h\right) g_{j}(t+h) \leq 0\right\}, t, h \geq 0,(3.9) \\
e \bigcup_{t \in \mathbb{R}_{+}} A_{t}=\Omega \text {, estão satisfeitas, ou seja, vale a condiçãao (3.5). }
\end{gathered}
$$

Prova: Desde que para todo $\omega \in \Omega$ e todo $t \in \mathbb{R}_{+}$, exista um $\gamma \subseteq\{1, \ldots, m\}$ tal que

$$
\sum_{j=1}^{m} I\left(T_{j}>t\right) g_{j}(t)=\sum_{j \in \gamma} g_{j}(t)
$$


então os eventos $A_{t}$ e $\left\{\sum_{j \in \gamma} g_{j}(t) \leq 0\right\}$ são equivalentes, para algum $\gamma \subseteq\{1, \ldots, m\}$.

Pela condição (3.8) temos

$$
\begin{aligned}
\left\{\sum_{j \in \gamma} g_{j}(t) \leq 0\right\} & \subseteq\left\{\sum_{j \in \gamma} g_{j}(t+h) \leq 0\right\} \\
& \subseteq\left\{\sum_{j=1}^{m} I\left(T_{j}>t+h\right) g_{j}(t+h) \leq 0\right\} .
\end{aligned}
$$

Assim $A_{t} \subseteq A_{t+h}$ para todo $t, h \geq 0$.

Para mostrar que $\bigcup_{t \in \mathbb{R}_{+}} A_{t}=\Omega$, defina a seguinte classe de eventos

$$
B_{s}=\left\{\omega: T=\max \left\{T_{1}, \ldots, T_{m}\right\} \leq s\right\},
$$

note que, como $T<\infty \quad$ P-q.c. então $B_{s} \neq \emptyset$, além disso, $\bigcup_{t \in \mathbb{R}_{+}} B_{t}=\Omega$, e mais $B_{s} \subseteq A_{s}$ para todo $s>0$, portanto $\bigcup_{t \in \mathbb{R}_{+}} A_{t}=\Omega$.

Proposição 3.6. Se $g_{j}, j=1, \ldots, m$, definidas como em (3.7), satisfaz a condição (3.8) então

$$
\sigma=\inf \left\{t \in \mathbb{R}_{+}: \sum_{j=1}^{m} I\left(T_{j}>t\right) g_{j}(t) \leq 0\right\}
$$

é o regra de parada ótima, isto é,

$$
E K_{\sigma}^{1}=\sup \left\{E K_{\tau}^{1}: \tau \Im_{t}-\text { tempo de parada }\right\}
$$

e portanto

$$
\sigma^{*}=\inf \left\{t \in \mathbb{R}_{+}: \sum_{j=1}^{m} I\left(T_{j}>t\right) g_{j}(t) \leq 0\right\} \wedge T
$$


é o tempo ótimo de burn-in para o problema 1.

Prova: Temos $K_{t}^{1}$ é SMR cuja a parte martingal $L_{t}$ é u.i.. Pelo Teorema 3.5 a condição de monotocidade é valida e podemos aplicar o Teorema 3.4 e a proposição esta provada.

Os seguintes casos especiais ilustram o resultado da Proposição 3.6.

\section{Observações 3.7.}

(i)Burn-in sempre.

Se $g_{j}(t)>0$ para todo $t \in \mathbb{R}_{+}, j=1, \ldots, m$, então $\sigma^{*}=T=\max \left\{T_{1}, \ldots, T_{m}\right\}$, i.e., o teste é realizado até que todos os itens falhem.

(ii) Não burn-in.

Se $g_{j}(0) \leq 0, j=1, \ldots, m$, então $\sigma^{*}=0$. Este caso sempre ocorre se $C_{B}=C_{F}$.

(iii) Itens Idênticos.

Se as taxas de falhas coincidem, i.e., $\lambda_{1}^{0}(t)=\ldots=\lambda_{m}^{0}(t)$ e $\lambda_{1}^{1}(t)=\ldots=\lambda_{m}^{1}(t)$ para todo $t \geq 0$, então $g_{j}(t)=g_{1}(t)$ para todo $j=1, \ldots, m$ e a condição (3.8) se 
reduz a

$$
g_{1}(s) \leq 0 \text { para } s \geq t_{1}=\inf \left\{t \in \mathbb{R}_{+}: g_{1}(t) \leq 0\right\}
$$

Se esta condição é satisfeita, o tempo de parada ótimo é da forma $\sigma^{*}=t_{1} \wedge$ $\max \left\{T_{1}, \ldots, T_{m}\right\}$.

(iv) O caso exponencial.

Se as taxas de falhas são constantes, iguais a $\lambda_{j}^{0}$ e $\lambda_{j}^{1}$, respectivamente, então $\mu_{j}$ e portanto $g_{j}$ são constantes, e $\sigma^{*}(\omega) \in\left\{0, T_{1}(\omega), \ldots, T_{m}(\omega)\right\}$, pois a condição (3.8) é satisfeita. Se além disso os itens são idênticos então temos que $\sigma^{*}=0$ ou $\sigma^{*}=\max \left\{T_{1}, \ldots, T_{m}\right\}$

(v)Troca de níveis de informação.

Se considerarmos uma subfiltragem $\left(\mathbb{A}_{t}\right)_{t \geq 0}$ de $\left(\Im_{t}\right)_{t \geq 0}$, onde $T$ é $\mathbb{A}_{t}$-adaptado, então o teorema da projeção (veja Teorema A.12) pode ser aplicado ao processo $K_{t}^{1}$ definido em (3.6) e obtemos assim um novo processo $\mathbb{A}_{t}$-mensuravel

$$
\begin{aligned}
\hat{K}_{t} & =X_{0}-Z_{0} \\
& +\int_{0}^{t} \sum_{j=1}^{m} E\left[I\left(T_{j}>s\right) \mid \mathbb{A}_{s}\right]\left[C \left(-1-\mu_{j}(s)\left(\lambda_{j}^{0}(s)-\lambda_{j}^{1}(s)\right)\right.\right. \\
& \left.+\left(C_{F}-C_{B}\right) \lambda_{j}^{0}(s)\right] d s+E\left[L_{t} \mid \mathbb{A}_{t}\right] .
\end{aligned}
$$

Podemos reescrever a Proposição 3.6 trocando

$$
\sigma^{*}=\inf \left\{t \in \mathbb{R}_{+}: \sum_{j=1}^{m} I\left(T_{j}>t\right) g_{j}(t) \leq 0\right\} \wedge T,
$$


por

$$
\hat{\sigma}^{*}=\inf \left\{t \in \mathbb{R}_{+}: \sum_{j=1}^{n} E\left[I\left(T_{j}>t\right) \mid \mathbb{A}_{t}\right] g_{j}(t) \leq 0\right\} \wedge T .
$$

Como exemplos, sejam:

(a) Se $\mathbb{A}_{t}=\sigma\{I(T>s), s \leq t\}$, então

$$
\hat{\sigma}^{*}=\inf \left\{t \in \mathbb{R}_{+}: \sum_{j=1}^{n} P\left(T_{j}>t\right) g_{j}(t) \leq 0\right\} \wedge T .
$$

(b) Se $\mathbb{A}_{t}=\sigma\left\{I(T>s), I\left(T_{i}>s\right), i \in \pi \subset\{1,2, \ldots, m\}, s \leq t\right\}$, então

$$
\hat{\sigma}^{*}=\inf \left\{t: \sum_{j \in \pi^{c}} P\left(T_{j}>t\right) g_{j}(t)+\sum_{j \in \pi} I\left(T_{j}>t\right) g_{j}(t) \leq 0\right\} \wedge T
$$

Exemplo 3.8. Se os tempos de vida (antes e depois do estresse) são exponenciais, com taxas de falhas $\lambda_{j}^{0}(t)=\lambda_{j}^{0}$ (constantes) e $\lambda_{j}^{1}(t)=\lambda_{j}^{1}$ (constantes) e $\lambda_{j}^{0}>\lambda_{j}^{1}, j=$ $1, \ldots, m$, temos:

$$
\mu_{j}(t)=\frac{\int_{t}^{\infty} e^{-\lambda_{j}^{1} s} d s}{e^{-\lambda_{j}^{1} t}}=\frac{1}{\lambda_{j}^{1}}
$$

$e$

$$
g_{j}(t)=g_{j}=\left(C_{F}-C_{B}\right) \lambda_{j}^{0}-\frac{C \lambda_{j}^{0}}{\lambda_{j}^{1}} .
$$

Para $m=2$, analisamos as seguintes condições

C1. Se $g_{1}+g_{2}>0$ então temos o seguinte:

i) Se $g_{2} \leq 0$ e $g_{1}>0$ temos $\lambda_{2}^{1} \leq \frac{C}{C_{F}-C_{B}}$ e $\lambda_{1}^{1}>\frac{C}{C_{F}-C_{B}}$. Aplicando a Proposição 3.6 obtemos $\sigma^{*}=T_{1} I\left(T_{1}<T_{2}\right)+T I\left(T_{1}>T_{2}\right)$ o tempo ótimo de burn-in.

ii) Se $g_{2}>0$ e $g_{1} \leq 0$ temos $\lambda_{2}^{1}>\frac{C}{C_{F}-C_{B}}$ e $\lambda_{1}^{1} \leq \frac{C}{C_{F}-C_{B}}$. Aplicando a Proposição 3.6 obtemos $\sigma^{*}=T_{2} I\left(T_{1}>T_{2}\right)+T I\left(T_{1}<T_{2}\right)$ o tempo ótimo de burn-in.

iii) $S e g_{2}>0$ e $g_{1}>0$ temos $\lambda_{2}^{1}>\frac{C}{C_{F}-C_{B}}$ e $\lambda_{1}^{1}>\frac{C}{C_{F}-C_{B}}$. Aplicando a Proposição 3.6 obtemos que $\sigma^{*}=T=\max \left\{T_{1}, T_{2}\right\}$ é o tempo ótimo de burn-in. 
C2. Há somente um caso a considerar quando $g_{1}+g_{2} \leq 0$ de forma que se satisfaça a condição (3.8). Neste caso, $g_{1} \leq 0$ e $g_{2} \leq 0$, e o tempo ótimo de burn-in é $\sigma^{*}=0$.

\subsubsection{Uma Solução para o Problema 2}

No problema 2, desejamos encontrar um $\Im_{t}$-tempo de parada ótimo $\sigma \in C_{T}^{\Im}$ satisfazendo

$$
K_{*}=E K_{\sigma}^{2}=\inf \left\{E K_{\tau}^{2}: \tau \in C_{T}^{\mathrm{F}}\right\}
$$

onde $K_{\tau}^{2}=\frac{E Z_{\tau}}{E X_{\tau}}$

Em outras palavras, a qualquer tempo $t$ o observador decide se para ou continua com o procedimento de burn-in baseado nas informações disponíveis até aquele instante.

Considerando que as relações entre variáveis aleatórias reais são $P$-q.c. uma primeira etapa para resolver este problema de otimização é estabelecer limites para $K_{*}$ em (3.13) e estes limites são dados pela seguinte proposição:

Proposição 3.9. Seja

$$
q=\sup \left\{\frac{f(t, \omega)}{g(t, \omega)}: 0 \leq t<T(\omega), \omega \in \Omega\right\}
$$

onde

$$
f(t, \omega)=\left(C_{B}-C_{F}\right) \sum_{j=1}^{n} I\left(T_{j}(\omega)>t\right) \lambda_{j}^{0}(t)<0,
$$

$e$

$$
g(t, \omega)=C \sum_{j=1}^{n} I\left(T_{j}(\omega)>t\right)\left[-1-\mu_{j}(t)\left(\lambda_{j}^{0}(t)-\lambda_{j}^{1}(t)\right)\right]<0 .
$$


Então $b_{l} \leq K^{*} \leq b_{u}$, onde

$$
\begin{gathered}
b_{u}=\frac{E Z_{0}}{E X_{0}} e \\
b_{l}= \begin{cases}0 & \text { se } E\left[Z_{0}-q X_{0}\right]<0 \\
\frac{E Z_{0}}{E X_{0}} & \text { se } E\left[Z_{0}-q X_{0}\right] \geq 0 .\end{cases}
\end{gathered}
$$

\section{Prova:}

Como $0 \in C_{T}^{\mathbb{E}}$ devemos encontrar somente a cota inferior $b_{l}$. Como $M, N$ são martingais u.i. e de médias zero, o teorema da parada opcional para martingal nos garante que $E M_{\tau}=E N_{\tau}=0$ para todo $\tau \in C_{T}^{\mathbb{F}}$.

Note que $q \geq 0$ pois $f(t, \omega)<0$ e $g(t, \omega)<0$, (veja (3.14) e (3.15)), e assim

$$
\begin{aligned}
q E X_{\tau}-q E X_{0} & =q E\left[\int_{0}^{\tau} g(s, \omega) d s\right]=E\left[\int_{0}^{\tau} q g(s, \omega) d s\right] \\
& \leq E\left[\int_{0}^{\tau} \frac{f(s, \omega)}{g(s, \omega)} g(s, \omega) d s\right]=E\left[\int_{0}^{\tau} f(s, \omega) d s\right] .
\end{aligned}
$$

Portanto, para todo $\tau \in C_{T}^{\mathrm{F}}, \tau<T$ temos

$$
E K_{\tau}^{2} \geq \frac{E Z_{0}+q E X_{\tau}-q E X_{0}}{E X_{\tau}}=\frac{E\left[Z_{0}-q X_{0}\right]}{E X_{\tau}}+q .
$$

Usando o fato de que $E X_{0} \geq E X_{\tau}$ para todo $\tau \in C_{T}^{\mathrm{F}}$ e que 0 é uma cota inferior para $E K_{t}^{2}$, prova-se a tese.

Como uma consequência imediata temos o corolário:

Corolário 3.10. Se $0 \leq q \leq \frac{n C_{F}}{C \sum_{j=1}^{n} \mu_{j}(0)}$ então

$$
K_{*}=\frac{n C_{F}}{C \sum_{j=1}^{n} \mu_{j}(0)}
$$


e $\sigma^{*}=0$ é o tempo ótimo de burn-in.

Para resolvermos o problema de otimização (3.13) utilizando a regra de parada IOPF é necessário transformá-lo em um problema de maximização. Uma técnica bem conhecida que transforma um problema de minimização em um problema equivalente de maximização é a seguinte: Note que $K_{\tau}^{2}=\frac{E Z_{\tau}}{E X_{\tau}} \geq K_{*}$ é equivalente a $K_{*} E X_{\tau}-E Z_{\tau} \leq 0$ para todo $\tau \in C_{T}^{\mathbb{F}}$, onde a igualdade é obtida com o tempo de parada ótimo.

Assim, devemos encontrar $\sigma \in C_{T}^{\mathbb{F}}$ com

$$
E H_{\sigma}=\sup \left\{E H_{\tau}: \tau \in C_{T}^{\mathbb{F}}\right\}=0,
$$

onde $H_{t}=K_{*} X_{t}-Z_{t}$ e $K_{*}=\inf \left\{K_{\tau}^{2}: \tau \in C_{T}^{\mathbb{F}}\right\}$.

Este novo problema de parada pode ser resolvido por meio da representação semimartingal do processo $H_{t}$. Para $t \in[0, T)$,

$$
H_{t}=K_{*} X_{0}-Z_{0}+\int_{0}^{t}\left(K_{*} g(s)-f(s)\right) d s+R_{t},
$$

onde $R_{t}=K_{*} N_{t}-M_{t}$ é martingal de média zero e $f(s), g(s)$ são definidas como em (3.14) e (3.15) respectivamente.

Usaremos a condição de monotonicidade (3.5) para encontrar tal solução. Observe que se o integrando

$$
h_{s}=K_{*} g(s)-f(s)
$$

satisfizer as condições do caso monótono (3.5), então o Teorema 3.4 se aplica e o tempo de parada baseado na regra IOPF, $\sigma=\inf \left\{t \in \mathbb{R}_{+}: h_{t} \leq 0\right\} \wedge T$ é o tempo ótimo, desde que $R_{t}$ seja u.i..

Obviamente, pelo fato de que $g(s,)<$.0 e $f(s,)<$.0 para todo $\omega \in \Omega$ e $0 \leq$ $s<T(\omega)$, o caso monótono (3.5) é verdadeiro se a razão $r(t)=\frac{f(t)}{g(t)}$ é descrescente, 
com $r(0)>K_{*}$ e $\lim _{t \rightarrow \infty} r(t)<K_{*}$, um vez que os eventos $\left\{\omega: r(t) \leq K_{*}\right\}$ e $\{\omega:$ $\left.K_{*} g(t)-f(t) \leq 0\right\}$ são equivalentes.

Um generalização da noção de monotocidade é a seguinte:

Definição 3.11. Sejam $a, b \in \mathbb{R} \bigcup\{-\infty, \infty\}$ constantes,com $a \leq b$. Então a função $r: \mathbb{R}_{+} \mapsto \mathbb{R}$ é chamada

(i) $(a, b)$-crescente se para todo $t, h \in \mathbb{R}_{+}$

$$
r(t) \geq a \Rightarrow r(t+h) \geq r(t) \wedge b
$$

(ii) $(a, b)$-decrescente se para todo $t, h \in \mathbb{R}_{+}$

$$
r(t) \leq b \Rightarrow r(t+h) \leq r(t) \vee a .
$$

Em outras palavras, uma função $r(t)$ é $(a, b)$-decrescente se ela passa decrescendo pelos níveis $b$ e $a$ e nunca mais retornam a tais níveis. Entre o níveis $b$ e $a$ a função $r(t)$ é não crescente, ou seja, se $r(t) \leq x$ para algum $x \in[a, b]$ então, $r(t+h) \leq x$ para todo $h \geq 0$ (veja por exemplo a figura 3.1). Além disso, uma função $r(t)$ $(0,0)$-decrescente satisfaz a condição de monotocidade (3.5) se $r(\infty) \leq 0$ (P-q.c.).

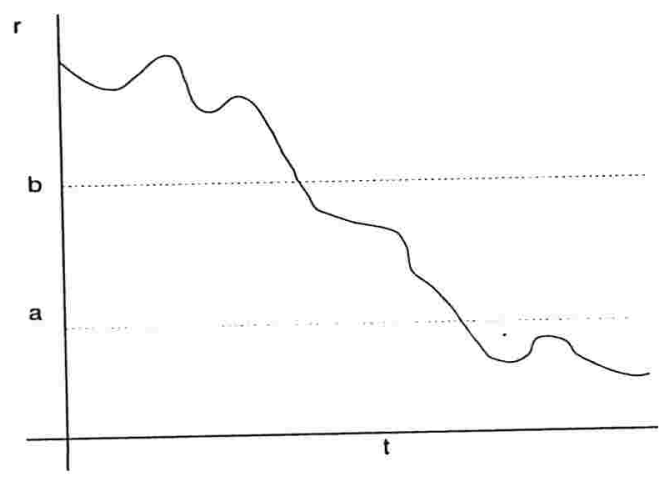

Figura 3.1: $r(t)(a, b)$-decrescente 
Proposição 3.12. Se $r(t)=\frac{f(t)}{g(t)}$ tem caminhos $\left(b_{l}, b_{u}\right)$-decrescente em $[0, T)$, então $x g(t)-f(t)$ satisfaz a condição de monotocidade (3.5) para todo $x \in\left[b_{l}, b_{u}\right]$.

Prova: Note que, para todo $x \geq 0$,

$$
r(t) \leq x \Leftrightarrow \frac{f(t)}{g(t)} \leq x
$$

Como $f(t, \omega)<0$ e $g(t, \omega)<0$ para todo $t \in[0, T)$ e para todo $\omega \in \Omega$, temos

$$
\frac{f(t)}{g(t)} \leq x \Leftrightarrow f(t) \geq x g(t) \Leftrightarrow x g(t)-f(t) \leq 0 .
$$

Além disso, como $r(t)=\frac{f(t)}{g(t)}$ tem caminhos $\left(b_{l}, b_{u}\right)$-decrescente em $[0, T)$, então $r(t)$ é $(x, x)$-decrescente para todo $x \in\left[b_{l}, b_{u}\right] e$

$$
\{w: r(t) \leq x\} \subseteq\{\omega: r(t+h) \leq x\}
$$

para todo $t, h \in \mathbb{R}_{+}$. Consequentemente

$$
\{\omega: x g(t)-f(t) \leq 0\} \subseteq\{\omega: x g(t+h)-f(t+h) \leq 0\}
$$

para todo $t, h \in \mathbb{R}_{+}$e a condição (3.5) é verdadeira para todo $x \in\left[b_{l}, b_{u}\right]$.

Proposição 3.13. Suponha que a função

$$
g_{j}(x, t)=-C x-C x \mu_{j}(t)\left(\lambda_{j}^{0}(t)-\lambda_{j}^{1}(t)\right)+\left(C_{F}-C_{B}\right) \lambda_{j}^{0}(t), \forall x \in\left[b_{l}, b_{u}\right],
$$

satisfaz a seguinte condição

$$
\sum_{j \in \gamma} g_{j}(x, t) \leq 0 \Rightarrow g_{j}(x, s) \leq 0, \forall j \in \gamma \subseteq\{1, \ldots, n\}, \forall s \geq t
$$


Então $r(t)=\frac{f(t)}{g(t)}$ tem caminhos $\left(b_{l}, b_{u}\right)$-decrescente em $[0, T)$.

Prova: Pelas definições de $f(t)$ e $g(t)$ (veja (3.14) e (3.15)), para todo $\omega \in \Omega$, para todo $t \in \mathbb{R}_{+}$e para todo $x \in\left[b_{l}, b_{u}\right]$ existe algum $\gamma \subseteq\{1,2, \ldots, m\}$ tal que

$$
x g(t)-f(t)=\sum_{j \in \gamma} g_{j}(x, t)
$$

Pela condição (3.21), temos que para todo $\gamma \subseteq\{1,2, \ldots, m\}$

$$
\sum_{j \in \gamma} g_{j}(x, t) \leq 0 \Rightarrow \sum_{j \in \gamma} g_{j}(x, t+h) \leq 0
$$

para $t, h \in \mathbb{R}_{+}$. Portanto para todo $\omega \in \Omega$ fixo,

$$
x g(t)-f(t) \leq 0 \Rightarrow x g(t+h)-f(t+h) \leq 0,
$$

para $t, h \in \mathbb{R}_{+}$, o que é equivalente a dizer que para todo $x \in\left[b_{l}, b_{u}\right]$ a função $r(t)=$ $\frac{f(t)}{g(t)}$ tem caminhos $(x, x)$-decrescente em $[0, T)$ e portanto é $\left(b_{l}, b_{u}\right)$-decrescente em $[0, T)$.

Defina

$$
\rho_{x}=\inf \{t: x g(t)-f(t) \leq 0\} \wedge T, \inf \emptyset=\infty, x \in\left[b_{l}, b_{u}\right]
$$

onde $f(t), g(t)$ são como em (3.14) e (3.15) respectivamente e $b_{l}, b_{u}$ são os limites inferiores e superiores de $K_{*}$ obtidas pela Proposição 3.9 O nível de parada ótimo $x_{*}$ para a razão $r(t)=\frac{f(t)}{g(t)}$ pode ser determinado mediante a equação $E H_{\sigma}=0 \mathrm{e}$ coincide com $K_{*}$ como demonstra o seguinte teorema: 
Teorema 3.14. Suponha que a função

$$
g_{j}(x, t)=-C x-C x \mu_{j}(t)\left(\lambda_{j}^{0}(t)-\lambda_{j}^{1}(t)\right)+\left(C_{F}-C_{B}\right) \lambda_{j}^{0}(t), \forall x \in\left[b l, b_{u}\right]
$$

satisfaz a condição (3.21).

Então, $\sigma=\sigma_{x_{*}}, \operatorname{com} x_{*}=\inf \left\{x \in\left[b_{l}, b_{u}\right]: x E X_{\sigma_{x}}-E Z_{\sigma_{x}}=0\right\}$ é o tempo ótimo de burn-in e $x^{*}=K_{*}$.

Prova: Pela Proposição 3.13 temos que $r(t)=\frac{f(t)}{g(t)}$ tem caminhos $\left(b_{l}, b_{u}\right)$ decrescente em $[0, T)$ e portanto pela Proposição 3.12 temos que $x g(t)-f(t)$ satisfaz a condição de monotocidade (3.5) para todo $x \in\left[b_{l}, b_{u}\right]$. Além disso, como $b_{l} \leq K_{*} \leq b_{u}$ e a parte martingal de $H_{t}=K_{*} X_{t}+Z_{t}$ é u.i., o tempo de parada ótimo é

$$
\sigma_{*}=\inf \left\{t: K_{*} g(t)-f(t) \leq 0\right\} \wedge T=\rho_{K_{*}}
$$

Para mostrar que $x_{*}=K_{*}$ basta observar que $\left.E H_{\sigma_{*}}=\sup \left\{E H_{\tau}: \tau \in C_{T}^{\mathrm{F}}\right\}=0\right\}$ e que $K_{*}=\inf \left\{E K_{\tau}^{2}: \tau \in C_{T}^{\mathrm{F}}\right\} \operatorname{com} K_{*} \in\left[b_{l}, b_{u}\right]$.

\section{Observações 3.15.}

(i)Burn-in sempre.

$\operatorname{Seg}(x, t)>0$ para $(x, t) \in\left[b_{l}, b_{u}\right] \times \mathbb{R}_{+}, j=1, \ldots, n$, então $\sigma_{*}=T=\max \left\{T_{1}, \ldots, T_{n}\right\}$, i.e., burn-in até que todos os itens falhem.

(ii) Não burn-in. 
Se $g(x, 0) \leq 0$ para $(x, 0) \in\left[b_{l}, b_{u}\right] \times \mathbb{R}_{+}, j=1, \ldots, n$, então $\sigma_{*}=0$.

(iii) Itens Idênticos.

Se as taxas de falhas se coincidem, i.e., $\lambda_{1}^{0}(t)=\ldots=\lambda_{n}^{0}(t)$ e $\lambda_{1}^{1}(t)=\ldots=\lambda_{n}^{1}(t)$ para todo $t \geq 0$, então $g_{j}(x, t)=g_{1}(x, t)$ para todo $j=1, \ldots, n$ e a condição (3.21) se reduz a

$$
g_{1}(x, s) \leq 0 \text { para } s \geq t_{1}=\inf \left\{t \in \mathbb{R}_{+}: g_{1}(x, t) \leq 0\right\}, x \in\left[b_{l}, b_{u}\right]
$$

(iv)Troca de níveis de informação.

Se considerarmos uma subfiltragem $\left(\mathbb{A}_{t}\right)_{t \geq 0}$ de $\left(\Im_{t}\right)_{t \geq 0}$, onde $T$ é $\mathbb{A}_{t}$ - adaptado, então o teorema da projeção pode ser aplicado sob o processo $H_{t}$ definido em (3.20) e obtemos assim um novo processo $\mathbb{A}_{t}$-mensuravel

$$
\begin{aligned}
\hat{H}_{t} & =K_{*} X_{0}-Z_{0} \\
& +\int_{0}^{t} \sum_{j=1}^{m} E\left[I\left(T_{j}>s\right) \mid \mathbb{A}_{s}\right]\left[K _ { * } C \left(-1-\mu_{j}(s)\left(\lambda_{j}^{0}(s)-\lambda_{j}^{1}(s)\right)\right.\right. \\
& \left.+\left(C_{F}-C_{B}\right) \lambda_{j}^{0}(s)\right] d s+E\left[R_{t} \mid \mathbb{A}_{t}\right] .
\end{aligned}
$$

Então podemos reescrever o Teorema 3.14 trocando

$$
\sigma_{x}=\inf \left\{t \in \mathbb{R}_{+}: \sum_{j=1}^{m} I\left(T_{j}>t\right) g_{j}(x, t) \leq 0\right\} \wedge T,
$$


por

$$
\hat{\sigma}_{x}=\inf \left\{t \in \mathbb{R}_{+}: \sum_{j=1}^{n} E\left[I\left(T_{j}>t\right) \mid \mathbb{A}_{t}\right] g_{j}(x, t) \leq 0\right\} \wedge T .
$$

Veja por exemplo:

(a) Se $\mathbb{A}_{t}=\sigma\{I(T>s), s \leq t\}$, então

$$
\hat{\sigma}_{x}=\inf \left\{t \in \mathbb{R}_{+}: \sum_{j=1}^{n} P\left(T_{j}>t\right) g_{j}(x, t) \leq 0\right\} \wedge T .
$$

(b) Se $\mathbb{A}_{t}=\sigma\left\{I(T>s), I\left(T_{i}>s\right), i \in \pi \subset\{1,2, \ldots, m\}, s \leq t\right\}$, então

$$
\hat{\sigma}_{x}=\inf \left\{t: \sum_{j \in \pi^{c}} P\left(T_{j}>t\right) g_{j}(x, t)+\sum_{j \in \pi} I\left(T_{j}>t\right) g_{j}(x, t) \leq 0\right\} \wedge T .
$$

Exemplo 3.16. Sejam $m=2$ itens produzidos e submetidos ao teste (procedimento) de burn-in (condição de forte estresses) com taxas de falhas exponenciais dadas por: $\lambda_{j}^{0}(t)=\lambda_{j}^{0}$ (taxa de falha do item $j$ durante o procedimento) e $\lambda_{j}^{1}(t)=\lambda_{j}^{1}$ (taxa de falha do item $j$ depois do procedimento), com $\lambda_{j}^{0}>\lambda_{j}^{1}, j=1,2$. Assim pela Proposição 3.9 os limites para $K_{*}$ é dado por $b_{u}=\frac{2 C_{F} \lambda_{1}^{1} \lambda_{2}^{1}}{C\left(\lambda_{1}^{1}+\lambda_{2}^{1}\right)} e$

$$
b_{l}= \begin{cases}0 & \text { se } q>b_{u} \\ b_{u} & \text { c.c. } .\end{cases}
$$

onde $q=\max \left\{A_{1,2}, A_{1}, A_{2}\right\}$

$$
A_{1,2}=\frac{\left(C_{F}-C_{B}\right)\left(\lambda_{1}^{0}+\lambda_{2}^{0}\right) \lambda_{1}^{1} \lambda_{2}^{1}}{C\left(\lambda_{1}^{0} \lambda_{2}^{1}+\lambda_{2}^{0} \lambda_{1}^{1}\right)}, A_{1}=\frac{\left(C_{F}-C_{B}\right) \lambda_{1}^{1}}{C}, \text { e } A_{2}=\frac{\left(C_{F}-C_{B}\right) \lambda_{2}^{1}}{C} .
$$

Para evitarmos o caso trivial $\left(b_{u}=b_{l}\right)$, vamos supor que $q>b_{u}$.

Se $b_{u}<\min \left\{A_{1,2}, A_{2}, A_{1}\right\}$, temos $r(t)=\frac{f(t)}{g(t)}>b_{u}$ onde $f(t)=\left(C_{B}-\right.$ $\left.C_{F}\right)\left(\lambda_{1}^{0} I\left(T_{1}>t\right)+\lambda_{2}^{0} I\left(T_{2}>t\right)\right)$ e $g(t)=-C\left(\frac{\lambda_{1}^{0} I\left(T_{1}>t\right)}{\lambda_{1}^{1}}+\frac{\lambda_{2}^{0} I\left(T_{2}>t\right)}{\lambda_{2}^{1}}\right) \mathrm{com}$ $C_{F}>C_{B}$, e portanto $x g(t)-f(t)>0$ para todo $x \in\left[b_{l}, b_{u}\right]$ e para todo $t \in[0, T)$. Portanto nestas condições temos que $\rho_{x}=T$ para todo $x \in\left[b_{l}, b_{u}\right]$ e $x_{*}=\inf \{x \in$ $\left.\left[b_{l}, b_{u}\right]: x E X_{\sigma_{x}}-E Z_{\sigma_{x}}=0\right\}=\inf \left\{x \in\left[b_{l}, b_{u}\right]: x=\frac{E Z_{T}}{E X_{T}}\right\}$. 
Se $A_{1} \leq b_{u}<\min \left\{A_{1,2}, A_{2}\right\}$ temos que

$$
\rho_{x}= \begin{cases}T_{2} I\left(T_{2}<T_{1}\right)+T I\left(T_{2}>T_{1}\right) & \text { se } A_{1} \leq x \leq b_{u} \\ T & \text { se } 0 \leq x<A_{1}\end{cases}
$$

Se $A_{2} \leq b_{u}<\min \left\{A_{1,2}, A_{1}\right\}$ temos que

$$
\rho_{x}= \begin{cases}T_{1} I\left(T_{2}>T_{1}\right)+T I\left(T_{2}<T_{1}\right) & \text { se } A_{2} \leq x \leq b_{u} \\ T & \text { se } 0 \leq x<A_{2} .\end{cases}
$$

Observe que em geral, o calculo de $x_{*}$ só é possivel através de simulação. 


\section{Apêndice A}

\section{Algumas Definições}

Neste capítulo apresentamos algumas definições e algums resultados relativos à teoria de processos estocásticos e da teoria clássica de confiabilidade em duas seções. Na primeira seção denominada por "Modelos Estocásticos de Falhas" abordamos um pouco da teoria de processos estocástico com enfoque em processos de contagem e suas particularidades. Estes resultados podem ser obtidos nas seguintes referências, Aven e Jensen (1999), Bremaud (1982) e em Dellacherie e Meyer (1980). Na segunda seção denominada por "Definições em Confiabilidade" apresentamos algums resultados clássicos em Teoria da Confiabilidade usando como referência o livro "Statistical Theory of Reliability and Life Testing: Probability Models" escrito por Barlow e Proschan (1981).

\section{A.1 Modelos Estocásticos de Falhas}

Seja $(\Omega, \Im, P)$ um espaço básico de probabilidade. A informação no instante $t$ é dada pela pré-t-história $\Im_{t}$. A filtragem $\mathbf{F}=\left(\Im_{t}\right)_{t \geq 0}$ é uma familia crescente de pré-t-história onde assumimos as seguintes condições, conhecidas como "condições usuais de Dellacherie": $\Im_{t} \subset \Im$ contém todos os $P$-conjuntos de medida nula de 
$\Im$ e $\Im_{t}=\Im_{t^{+}}=\cap_{s>t} \Im_{s}$, i.e. $\Im_{t}$ são contínuas à direita e completas. Além disso definimos $\Im_{\infty}=\bigvee_{t \geq 0} \Im_{t}$ como a menor $\sigma$-algebra que contem todos os eventos de $\Im_{t}$ para todo $t \in \mathbb{R}_{+}$.

Por simplicidade de notação, vamos assumir ao longo de todo o texto que as relações como $C,=, \leq,<,=$ entre conjuntos mensuraveis e variáveis aleatórias, respectivamente, sempre serão com probabilidade um, com isso o termo $P$-q.c. será omitido. Para esperanças condicionais não vamos fazer diferença entre uma versão e a classe de equivalência $P$-q.c. dessas versões.

\section{Definição A.1. (Processos Progressivos)}

Um processo $X=\left(X_{t}\right)_{t \geq 0}$ é $\mathbf{F}$-progressivo ou progressivamente mensurável, se para todo $t$ a aplicação $(s, \omega) \rightarrow X_{s}(\omega) \operatorname{com}(s, \omega) \in[0, t] \times \Omega$ é mensurável com respeito a $\sigma$-álgebra $\mathbb{B}([0, t]) \otimes \Im_{t}$, onde $\mathbb{B}([0, t])$ é a $\sigma$-álgebra de Borel em $[0, t]$.

Pode-se provar que todo processo adaptado, contínuo à esquerda ou à direita é progressivamente mensurável (Dellacherie e Meyer (1980)). Se X é progressivo, então $Y=\left(Y_{t}\right), Y_{t}=\int_{0}^{t} X_{s} d s$ também é progressivo.

Um conceito fundamental na teoria geral de processos estocásticos é o de previsibilidade:

\section{Definição A.2. (Processos Previsiveis)}

Seja $\mathbf{F}$ uma filtragem em um espaço de probabilidade básico e seja $\mathbb{P}(\mathbf{F})$ a $\sigma$ álgebra em $(0, \infty) \times \Omega$ gerada pelos sistemas de conjuntos

$$
(s, t] \times A, 0 \leq s<t, A \in \Im_{s}, t>0 .
$$


$\mathbb{P}(\mathbf{F})$ é chamada de $\sigma$-álgebra $\mathbf{F}$-previsivel em $(0, \infty) \times \Omega$. Um processo $X=\left(X_{t}\right)$ é chamado F-previsível, se $X_{0}$ é $\Im_{0}$-mensurável e a aplicação $(t, \omega) \rightarrow X_{t}(\omega)$ de $(0, \infty) \times \Omega$ em $\mathbb{R}$ é mensurável com respeito a $\mathbb{P}(\mathbf{F})$.

Todo processo adaptado e contínuo à esquerda é previsível e todo processo Fprevisível é F-progressivo.

Para obtermos o significado do termo previsível, observamos que para um processo $X \quad$ F-previsível, o valor $X_{t}$ pode ser previsto mediante informações disponíveis antes do tempo $t$, isto é, $X_{t}$ é mensurável com respeito a $\Im_{t-}=\bigvee_{s<t} \Im_{s}=\sigma\left\{A_{s}, A_{s} \in\right.$ $\left.\Im_{s}, 0 \leq s<t\right\}$. Processos desse tipo são elementos muito importante na teoria dos processos pontuais.

Definição A.3. (Semimartingais Regulares)

Um processo estocástico $Z=\left(Z_{t}\right)_{t \leq 0}$ é chamado um semimartingal regular ("smooth semimartingale") se ele tem um decomposição da forma

$$
Z_{t}=Z_{0}+\int_{0}^{t} f_{s} d s+M_{t}
$$

onde $f=\left(f_{t}\right)_{t \geq 0}$ é um processo estocástico progressivamente mensurável com

$$
E\left[\int_{0}^{t}\left|f_{s}\right| d s\right]<\infty
$$

para todo $t \in \mathbb{R}_{+}, E\left|Z_{0}\right|<\infty, M=\left(M_{t}\right)_{t \geq 0}$ em um martingal contínuo à direita com limites à esquerda e $M_{0}=0$.

Em geral usa-se a notação abreviada: $Z=(f, M)$. 


\section{Definição A.4. (Processos Pontuais "Univariados)}

Um processo pontual(univariado) é uma sequência crescente de variáveis aleatórias positivas $\left(T_{n}\right), n \in\{0,1, \ldots\}$ que podem assumir valores $+\infty: 0 \leq T_{1} \leq T_{2} \leq \ldots$ As desigualdades são estritas a menos que $T_{n}=\infty$. Vamos assumir que o processo é não explosivo, isto é,

$$
T_{\infty}=\lim _{n \rightarrow \infty} T_{n}=\infty
$$

Note que o processo pontual é completamente caracterizado pela medida aleatória $\mu$ em $(0, \infty)$ definida por

$$
\mu(\omega, A)=\sum_{k \geq 1} I\left(T_{k}(\omega) \in A\right)
$$

para todo conjunto de Borel $A \in(0, \infty)$.

Um maneira equivalente para descrever um processo pontual é pelo processo de contagem $N=\left(N_{t}\right)_{t \geq 0}$, com

$$
N_{t}(\omega)=\sum_{k \geq 1} I\left(T_{k}(\omega) \leq t\right)
$$

que é, para cada realização $\omega$, um função escada contínua à direita com saltos de tamanho 1 e $N_{0}(\omega)=0 . N_{t}$ conta os números de pontos $T_{n}$, que ocorrem até o instante $t$. Em geral dizemos que o processo de contagem associado $N$ é um processo pontual.

Uma generalização deste conceito é o de processo pontual multivariado. Se $\left(T_{n}\right), n \in\{1,2, \ldots\}$ é um processo pontual e $\left(V_{n}\right), n \in\{1, \ldots\}$, uma sequência de variáveis aleatórias com valores no conjunto finito $\left\{a_{1}, \ldots, a_{m}\right\}$, então a sequência de pares $\left(T_{n}, V_{n}\right), n \in\{1,2, \ldots\}$ é chamado de processo pontual multivariado. $O$ processo $N_{t}=\left(N_{t}(1), \ldots, N_{t}(m)\right)$ onde,

$$
N_{t}(i)=\sum_{k \geq 1} I\left(T_{k}(\omega) \leq t\right) I\left(V_{k}=a_{i}\right), i \in\{1, \ldots, m\}
$$


é o processo de contagem $m$-variado associado.

Dado um processo pontual univariado $\left(T_{n}\right), n \in\{1,2, \ldots\}$ e o processo de contagem associado $\left(N_{t}\right)_{t \geq 0}$, com $E N_{t}<\infty$ para todo $t \in \mathbb{R}_{+}$em um espaço de probabilidade filtrado $(\Omega, \Im, \mathbf{F}, P)$, podemos considerar a definição de seu compensador:

\section{Definição A.5. (Compensador)}

Seja $N$ um processo de contagem integrável, adaptado a uma filtragem $\mathbf{F}$. O único processo crescente $\mathbf{F}$-previsivel $A=\left(A_{t}\right)_{t \geq 0}$, tal que

$$
E \int_{0}^{\infty} C_{s} d N_{s}=E \int_{0}^{\infty} C_{s} d A_{s}
$$

é verdadeiro para todo processo não negativo $\mathbf{F}$-previsivel $C$, é chamado o compensador de $N$ com respeito $a \mathbf{F}$.

Teorema A.6. (Dellacherie e Meyer (1980))

Se $N$ é um processo pontual integrável, adaptado a filtragem $\mathbf{F}$. Então $A$ é o $\mathbf{F}$-compensador de $N$ se e somente se a diferença $N-A$ é um $\mathbf{F}$-martingal com média zero.

Se considerarmos o compensador como uma medida aleatória $A(d t)$ em $(0, \infty)$, então podemos interpretar esta medida na forma infinitesimal pela expressão heurística

$$
A(d t)=E\left[d N_{t} \mid \Im_{t-}\right]
$$

Em muito casos (na realidade os caso de nosso interesse) o $\mathbf{F}$-compensador de um processo de contagem pode ser representado como uma integral na forma

$$
A_{t}=\int_{0}^{t} \lambda_{s} d s
$$


onde $\left(\lambda_{t}\right)_{t \geq 0}$ é um processo estocástico não negativo $\mathbf{F}$-progressivo, isto é, $N$ tem uma representação $\mathrm{SSM}, N=(\lambda, M)$.

\section{Definição A.7. (Intensidade)}

Seja $N$ um processo de contagem integrável com uma $\mathbf{F}$-SMR representação (veja

$$
N_{t}=A_{t}+M_{t}=\int_{0}^{t} \lambda_{s} d s+M_{t}
$$

onde $\left(\lambda_{t}\right)_{t \geq 0}$ é um processo estocástico não negativo. Então $\lambda$ é chamado a $\mathbf{F}$ intensidade de $N$.

\section{Observações A.8.}

(i) Pode-se mostrar (veja Brémaud (1981)) que se a intensidade for previsível então ela é unica e por outro lado, se existe uma intensidade, então sempre podemos encontrar uma versão previsivel.

(ii) A interpretação heurística

$$
\lambda_{t} d t=E\left[d N_{t} \mid \Im_{t-}\right]
$$

é muito similar à interpretação da taxa de risco de uma variável aleatória positiva.

Considerando a filtragem baseada na história interna do processo de contagem $N$, isto é, $\mathbf{F}^{N}=\left(\Im_{t}^{N}\right)_{t \geq 0}, \Im_{t}^{N}=\sigma\left\{N_{s}, 0 \leq s \leq t\right\}$, Brémaud (1981) provou que:

Teorema A.9. (Brémaud (1981))

Seja $N$ um processo de contagem e $\mathrm{F}^{N}$ sua história interna. Para cada $n$ se $G_{n}(\omega, B)$ é distribuição condicional regular dos tempos entre as chegadas $U_{n+1}=$ 
$T_{n+1}-T_{n}, n \in\{0,1, \ldots\}, T_{0}=0$, dados o passado $\Im_{T_{n}}^{N}$ no $\mathbf{F}^{N}$-tempo de parada $T_{n}$, i.e., $G_{n}(\omega, B)=P\left(U_{n+1} \in B \mid \Im_{T_{n}}^{N}\right)(\omega)$.

(i) Então para $T_{n}<t \leq T_{n+1}$ o compensador $A$ é dado por

$$
A_{t}=A_{T_{n}}+\int_{0}^{t-T_{n}} \frac{G_{n}(d x)}{G_{n}([x, \infty))} .
$$

(ii) Se a distribuição condicional $G_{n}$ admite uma densidade $g_{n}$ para todo $n$, então $a \mathbf{F}^{N}$-intensidade $\lambda$ é dada por

$$
\lambda_{t}=\sum_{n \geq 0} \frac{g_{n}\left(t-T_{n}\right) .}{1-\int_{0}^{t-T_{n}} g_{n}(x) d x} I\left(T_{n}<t \leq T_{n+1}\right) .
$$

Exemplo A.10. (Processo de Renovação)

Se os tempos entres chegadas $U_{n+1}=T_{n+1}-T_{n}, n \in\{0,1, \ldots\}, T_{0}=0$, são variáveis aleatórias i.i.d. com função distribuição comum $F$, densidade $f$ e taxa de falha $r: r(t)=f(t) /(1-F(t))$, então, a sequência $\left(T_{n}\right)_{n \geq 1}$ determina um processo de renovação. Considerando $\Im_{t}^{N}=\sigma\left\{N_{s}, 0 \leq s \leq t\right\}, t \in \mathbb{R}_{+}$, e aplicando o Teorema A.9 temos que a intensidade em $\left\{T_{n}<t \leq T_{n+1}\right\}$ é dada por $\lambda_{t}=r\left(t-T_{n}\right)$. Deste resultado obtemos a representação $S M R, N=(\lambda, M)$,

$$
N_{t}=\int_{0}^{t} \lambda_{s} d s+M_{t}
$$

com intensidade

$$
\lambda_{t}=\sum_{n \geq 0} r\left(t-T_{n}\right) I\left(T_{n}<t \leq T_{n+1}\right)
$$

Definição A.11. (Subfiltragem)

Sejam $\mathbf{A}=\left(\mathcal{A}_{t}\right)_{t \geq 0}$ e $\mathbf{F}=\left(\Im_{t}\right)_{t \geq 0}$ duas filtragem em um mesmo espaço de probabilidade $(\Omega, \Im, P)$. Então A é chamada uma subfiltragem de $\mathbf{F}$ se $\mathcal{A}_{t} \subset \Im_{t}$ para todo $t \in \mathbb{R}_{+}$. 
Teorema A.12. (Teorema da Projeção, Jensen U. (1989))

Seja $Z=(f, M)$ um $\mathbf{F}-S M R$ e $\mathbf{A}$ uma subfiltragem de $\mathbf{F}$. Então $\widehat{Z}$ com

$$
\widehat{Z}_{t}=\widehat{Z}_{0}+\int_{0}^{t} \hat{f}_{s} d s+\bar{M}_{t}
$$

é um A-SMR, onde

(i) $\widehat{Z}$ é $\mathbf{A}$-adaptado com caminhos q.c. contínuos à direita, com limite à esquerda e $\widehat{Z}_{t}=E\left[Z_{t} \mid \mathcal{A}_{t}\right]$ para todo $t \in \mathbb{R}_{+}$;

(ii) $\hat{f}$ é A-progressivo com $\hat{f}_{t}=E\left[f_{t} \mid \mathcal{A}_{t}\right]$ para quase todo $t \in \mathbb{R}_{+}$(Medida de Lebesgue);

(iii) $\bar{M}$ é um A-martingal.

Se além disso, $Z_{0}, \int_{0}^{t}\left|f_{s}\right| d s \in L^{2}$ ( $f$ é quadrado integrável) e $M$ é um martingal quadrado integrável e de média zero, então $\widehat{Z}_{0}, \int_{0}^{t}\left|\hat{f}_{s}\right| d s \in L^{2}$ e $\bar{M}$ é um martingal quadrado integrável e de média zero.

O teorema da projeção é muito usado para resolver problemas de parada ótima em diferentes níveis de informação. Seja por exemplo, $Z=(f, M)$ é um F-SMR e se $\widehat{Z}=(\hat{f}, \bar{M})$ é o correspondente $\mathbf{A}$-SMR com respeito a subfiltragem $\mathbf{A}$ de $\mathbf{F}$. Para determinar o máximo de $E Z_{\tau}$ em um conjunto $C^{\mathbf{A}}$ de $\mathbf{A}$ - tempo de parada admissíveis $\tau$, isto é, para resolver um problema de parada ótima sob o nível de informação $\mathbf{A}$, podemos usar sucessivos condicionamento e através das esperanças condicionais obtermos

$$
\sup \left\{E Z_{\tau}: \tau \in C^{\mathbf{A}}\right\}=\sup \left\{E \widehat{Z}_{\tau}: \tau \in C^{\mathbf{A}}\right\}
$$

Seja $T$ o tempo de vida de um sistema definido em um espaço de probabilidade básico e $Z_{t}=I(T \leq t)$, seu indicador. Obviamente $Z$ é um processo de contagem 
correspondente ao processo pontual simples $\left(T_{n}\right)$ com $T_{1}=T$ em $T_{n}=\infty$ para $n \geq 2$. As trajetórias desse processo são constantes exceto no salto de 0 para $1 \mathrm{em}$ $T$. O modelo de tempo de vida geral é definido por

$$
I(T \leq t)=\int_{0}^{t} I(T \geq s) \lambda_{s} d s+M_{t}, t \in \mathbb{R}_{+},
$$

isto é, uma representação $\mathbf{F}$-semimartingal regular para o processo $Z_{t}$ com $\mathbf{F}$ martingal $M$ de média zero, e um processo estocástico não negativo $\lambda=\left(\lambda_{t}\right)$. O modelo de tempo de vida geral é então determinado pela filtragem $\mathbf{F}$ e a correspodente $\mathbf{F}$-SMR representando o processo indicador.

Definição A.13. (Processos de Riscos)

O processo $\lambda=\left(\lambda_{t}\right)_{t \geq 0}$ da SMR-representação (A.4) é chamado de processo taxa de risco (taxa de falha) com respeito a $\mathbf{F}$ e o compensador $\Lambda_{t}=\int_{0}^{t} I(T \geq s) \lambda_{s} d s$ é chamado de processo $\mathbf{F}$-risco.

Definição A.14. (Tempos de Paradas)

Uma variável aleatória positiva $\tau$ é chamada um $\mathbf{F}$-tempo de parada se $\{\tau \leq$ $t\} \in \Im_{t}$ para todo $t \in \mathbb{R}_{+}$. Um $\mathbf{F}$-tempo de parada $\tau$ é:

(i) previsivel se existe uma sequencia crescente $\left(\tau_{n}\right), n \in\{1,2, \ldots\}$ de tempos de paradas, $\tau_{n}<\tau$ que $\lim _{n \rightarrow \infty} \tau_{n}=\tau$;

(ii) totalmente inacessível se $P(\tau=\sigma<\infty)=0$ para todo tempo de parada $\sigma$ F-previsível. 
Lema A.15. (Dellacherie e Meyer (1980))

Seja $(\Omega, \Im, \mathbf{F}, P)$ um espaço de probabilidade filtrado e $T$ um $\mathbf{F}$-tempo de parada.

(i) Se o processo $V=\left(V_{t}\right), V_{t}=I(T \leq t)$, tem uma representação $S M R$

$$
V_{t}=\int_{0}^{t} I(T \geq s) \lambda_{s} d s+M_{t}, t \in \mathbb{R}_{+}
$$

então $T$ é um tempo de parada totalmente inacessivel e o martigal $M$ é quadrado integrável de média zero.

(ii) Se T é um tempo de parada totalmente inacessivel então o processo $V=$ $\left(V_{t}\right), V_{t}=I(T \leq t)$, tem uma unica (P-q.c.) decomposição $V=\Lambda+M$, onde $M$ é um martingal uniformemente integrável e $\Lambda$ é contínua (P-q.c., compensador previsivel).

O seguinte teorema mostra a relação entre a troca de medidas de probabilidade absolutamente contínuas e a troca das intensidades induzidas por estas. A transformação da medida $d N_{t} d P$ na medida $d N_{t} d Q$ onde $Q$ é absolutamente contínua em relação a $P$, através do Teorema de Radon-Nikodym (veja Chung (1968)) pode ser interpretada pela transformação nas intensidades $\lambda_{t} \rightarrow \widetilde{\lambda}_{t}$.

Teorema A.16. (Teorema de Girsanov, Brémaud (1982))

Seja $\left(N_{t}(1), \ldots, N_{t}(k)\right)$ um processo de contagem $k$-variado, adaptado a alguma história $\Im_{t}$, e seja $\lambda_{t}(i), i=1, \ldots, k$, as $\left(P, \Im_{t}\right)$-intensidades previsiveis de $N_{t}(i), i=$ $1, \ldots, k$, respectivamente. Sejam $\mu_{t}(i), i=1, \ldots, k$, processos $\Im_{t}$-previsíveis, não negativos tais que para todo $t \geq 0$ e todo $1 \leq i \leq k$

$$
\int_{0}^{t} \mu_{s}(i) \lambda_{s}(i) d s<\infty(P-q . c .)
$$


Defina o processo $L_{t}$ por

$$
L_{t}=\prod_{i=1}^{k} L_{t}(i)
$$

onde

$$
L_{t}(i)=\left(\prod_{n \geq 1} \mu_{T_{n}}(i) I\left(T_{n} \leq t, V_{n}=i\right)\right) \exp \left\{\int_{0}^{t}\left(1-\mu_{s}(i)\right) \lambda_{s}(i) d s\right\},
$$

onde $T_{n}$ é o tempo da n-ésima falha e $V_{n}$ sua respectiva marca. Então :

(i) $L_{t}$ é um $\left(P, \Im_{t}\right)$-martingal local;

(ii) se $E\left[L_{\infty}\right]=1$, podemos definir a medida de probabilidade $Q$ por

$$
\frac{d Q}{d P}=L_{\infty}
$$

então, para todo $i=1, \ldots, k, N_{t}(i)$ tem $a\left(Q, \Im_{t}\right)$-intensidade $\tilde{\lambda}_{t}(i)=\mu_{t}(i) \lambda_{t}(i)$.

Observações A.17. (i) Note que $L_{t}=E_{P}\left[\frac{d Q}{d P} \mid \Im_{t}\right]$. Se $L_{t}$ é u.i. então $L_{t}$ é um $\left(P, \Im_{t}\right)$-martingal;

(ii) Seja $\frac{d Q}{d P}=L_{\infty}$ a derivada de Radon-Nikodym, onde $E_{P}\left[L_{\infty}\right]=1$. Se $M=$ $\left(M_{t}\right)_{t \geq 0}$ é um $\left(P, \Im_{t}\right)$-martingal, então $\frac{M}{L}=\left(\frac{M_{t}}{L_{t}}\right)_{t \geq 0}$ é um $\left(Q, \Im_{t}\right)$-martingal. Da mesma forma, se $M=\left(M_{t}\right)_{t \geq 0}$ é um $\left(Q, \Im_{t}\right)$-martingal, então $M L=\left(M_{t} L_{t}\right)_{t \geq 0}$ é $u m\left(P, \Im_{t}\right)$-martingal.

Teorema A.18. (Representação integral para Martingais, Bremaud (1982))

Se $\left(N_{t}(1), \ldots, N_{t}(m)\right)$ é um processo pontual m-variado em $(\Omega, \Im, P), P$-não explosivo e seja $\Im_{t}^{N}$ a história interna do processo. Suponha que para cada $i, 1 \leq$ $i \leq m, N_{t}(i)$ admita a $\Im_{t}^{N}$-intensidade previsivel $\lambda_{t}(i)$. Se $M_{t}$ é um $\Im_{t}^{N}$-martingal 
contínuo à direita da forma $M_{t}=E\left[M_{\infty} \mid \Im_{t}^{N}\right]$ onde $M_{\infty}$ é alguma variável aleatória em $\Im, P$-integrável, ou $M_{t}$ é um martingal quadrado integrável, isto é,

$$
\sup _{t \geq 0} E\left|M_{t}\right|^{2}<\infty
$$

Então para todo $t \geq 0$, existem processos $\Im_{t}^{N}$-previsiveis $H_{t}(i), 1 \leq i \leq m$, satisfazendo

$$
\int_{0}^{t}\left|H_{s}(i)\right| \lambda_{s}(i) d s<\infty \quad P-q . c .
$$

tal que

$$
M_{t}=M_{0}+\sum_{i=1}^{m} \int_{0}^{t} H_{s}(i)\left(d N_{s}(i)-\lambda_{s}(i) d s\right) \quad P-q . c .
$$

\section{A Teoria da Inovação}

Consideremos um processo estocástico $X_{t}$, definido em um espaço de probabilidade $(\Omega, \Im, P)$, contínuo à direita com valores em $S=\{1, \ldots, m\}$ e duas histórias:

i) a história global $\Im_{t}=\Im_{t}^{N} \vee \Im_{t}^{X}$,

ii) a história observada $\Re_{t}=\Im_{t}^{N}$,

onde a história observada $\Im_{t}^{N}$ é a história interna de um processo pontual integrável $m$-variado $\left(N_{t}(1), \ldots, N_{t}(m)\right)$ com $\Im_{t}$-intensidade $\left(\lambda_{t}(1), \ldots, \lambda_{t}(m)\right)$ e $\Im_{t}^{N}$-intensidade $\left(\lambda_{t}^{p}(1), \ldots, \lambda_{t}^{p}(m)\right)$ onde $\Im_{t}$ é história global dos processos $\left(\Im_{t}=\Im_{t}^{X} \vee \Im_{t}^{N}, \Im_{t}^{N}=\right.$ $\left.\sigma\left\{N_{s}(i): 0 \leq s \leq t, i=1, \ldots, m\right\}\right)$.

Em geral o objetivo é encontrar expressões para o processo $E\left[h_{t} \mid \Re_{t}\right]$, onde $h_{t}$ é algum processo integrável adaptado a $\Im_{t}$. Nosso objetivo é encontrar uma expressão 
para

$$
Z_{t}=E\left[Y_{t} \mid \Im_{t}^{N}\right]
$$

onde $Y_{t}=h\left(X_{t}\right)$ é um processo integrável e $h$ é um função mensurável de $S$ com valores reais.

Note que se o processo $Y_{t}$ é quadrado integrável temos que $Z_{t}=E\left[Y_{t} \mid \Im_{t}^{N}\right]$ é o melhor estimador de $Y_{t}$ no tempo $t$, um vez que pelo criterio quadrático (veja Dellacherie e Meyer (1980)) temos

$$
E\left[\left|Y_{t}-E\left[Y_{t} \mid \Im_{t}^{N}\right]\right|^{2}\right] \leq E\left[\left|Y_{t}-W\right|^{2}\right]
$$

para qualquer variável aleatória quadrado integrável $W$ que é $\Im_{t}^{N}$-mensurável. Se admitimos que o processo $Y_{t}$ é limitado e tem uma representação $\Im_{t}$-semimartingal regular conforme a Definição $A .3$, ou seja

$$
Y_{t}=Y_{0}+\int_{0}^{t} f_{s} d s+m_{t}
$$

onde $f_{s}$ é um processo $\Im_{t}$-progressivo satisfazendo $E\left[\int_{0}^{t}\left|f_{s}\right| d s\right]<\infty, t \geq 0$, e $m_{t}$ é um martigal de média zero, concluimos, do teorema da projeção (Teorema A.12) que o processo $Z_{t}=E\left[Y_{t} \mid \Im_{t}^{N}\right]$ tem uma representação semimartingal regular em $\Im_{t}^{N}$.

Definição A.19. (Equações de ganho de Inovação)

Para cada $1 \leq i \leq m$, vamos denotar pelos processos $\psi_{1, t}(i), \psi_{2, t}(i)$, e $\psi_{3, t}(i), \Im_{t}^{N}$ previsiveis que satisfazem

$$
\begin{aligned}
E\left[\int_{0}^{t} C_{s} Y_{s} \lambda_{s}(i) d s\right] & =E\left[\int_{0}^{t} C_{s} \psi_{1, s}(i) \lambda_{s}^{p}(i) d s\right] \\
E\left[\int_{0}^{t} C_{s} Y_{s} \lambda_{s}^{p}(i) d s\right] & =E\left[\int_{0}^{t} C_{s} \psi_{2, s}(i) \lambda_{s}^{p}(i) d s\right] \\
E\left[\sum_{0 \leq s \leq t} C_{s} \Delta m_{s} \Delta N_{s}(i)\right] & =E\left[\int_{0}^{t} C_{s} \psi_{3, s}(i) \lambda_{s}^{p}(i) d s\right]
\end{aligned}
$$


onde $\left(C_{s}\right)_{s \geq 0}$ é $\Re_{s}$-prevísivel.

Dessas considerações acima podemos provar o seguinte teorema.

Teorema A.20. (Expressão do Ganho de Inovação, Brémaud (1982))

$\operatorname{Sejam} \Im_{t}=\Im_{t}^{X} \vee \Im_{t}^{N}, \Im_{t}^{N}=\sigma\left\{N_{s}(i): 0 \leq s \leq t, i=1, \ldots, m\right\}$ e $\Re_{t}=\Im_{t}^{N}$ as histórias global e observadas do processos pontual integrável $m$-variado

$$
\left(N_{t}(1), \ldots, N_{t}(m)\right)
$$

com $\Im_{t}$-intensidades $\left(\lambda_{t}(1), \ldots, \lambda_{t}(m)\right)$ e $\Re_{t}$-intensidades $\left(\lambda_{t}^{p}(1), \ldots, \lambda_{t}^{p}(m)\right)$ se o processo $Y_{t}$ é limitado e tem $\Im_{t}$-representação semimartingal (A.10), então o processo $Z_{t}=E\left[Y_{t} \mid \Im_{t}^{N}\right]$ tem a seguinte representação

$$
Z_{t}=E\left[Y_{0}\right]+\int_{0}^{t} E\left[f_{s} \mid \Im_{s}^{N}\right] d s+\sum_{i=1}^{m} \int_{0}^{t} K_{s}(i)\left(d N_{s}(i)-\lambda_{s}(i) d s\right) P-q . c .
$$

onde para todo $1 \leq i \leq m$

$$
K_{t}(i)(\omega)=\psi_{1, t}(i)(\omega)-\psi_{2, t}(i)(\omega)+\psi_{3, t}(i)(\omega)
$$

onde os $\psi(i)$ 's são definidos pela equação (A.11).

Observação A.21. Pela primeira equação de (A.11), temos que o processo

$$
\psi_{1, t}(i)=Z_{t-}
$$

Se $Y_{t}$ e $N_{t}(i)$ não tem saltos em comum, $\psi_{3, t}(i)=0$. 
Definição A.22. (Cadeia de Markov)

Um processo de saltos é um processo contínuo à direita com trajetorias contantes por partes. Seja $X=\left(X_{t}\right)_{t \geq 0}$ um processo de saltos definido em $(\Omega, \Im, P)$ tomando valores em $S=\{1,2, \ldots, m\}$ ou em $S=\{1,2, \ldots\}$ e adaptado a filtragem natural do processo $\Im_{t}^{X}=\sigma\left\{X_{s}, 0 \leq s \leq t\right\}$. Então $\left(X_{t}\right)_{t \geq 0}$ é dito uma cadeia de markov com valores no espaço de estados $S$ se

$$
P\left(X_{t}=i \mid \Im_{s}, s \leq r\right)=P\left(X_{t}=i \mid \Im_{r}\right), q . c .
$$

para todo $0 \leq r \leq t$ e $i \in S$.

Observação A.23. Em geral usa-se a seguinte notação para as probabilidades condicionais definidas em (A.15).

$$
P\left(X_{t}=i \mid X_{s}, s \leq r\right)=P\left(X_{t}=i \mid X_{r}\right), q . c . .
$$

Para todo $i, j \in S$ e $0 \leq s \leq t$, denotaremos por

$$
p_{i j}(t, s)=P\left(X_{t}=j \mid X_{s}=i\right)
$$

a probabilidade de transição de $i$ para $j$.

Definição A.24. (q-Propriedade)

Seja $Q(t)=\left(q_{i j}(t)\right), i, j \in S$ uma matriz. Dizemos que $Q(t)$ satisfaz a $q$ Propriedade se:

(i) $q_{i j}(t)$ é Borel mensuravel para todo $i, j \in S$ e $t \geq 0$.

(ii) $q_{i j}(t)$ é uniformemente limitada, isto é, existe uma constante $K$ tal que $\left|q_{i j}(t)\right| \leq K, \forall i, j \in S$, e $t \geq 0$.

(iii) $q_{i j}(t) \geq 0$ para $j \neq i$, e $q_{i i}(t)=\sum_{j \neq i} q_{i j}(t), t \geq 0$. 
Para qualquer função $f$ de valor real definida em $S$ e $i \in S$ defina o seguinte operador:

$$
Q(t) f(.)(i)=\sum_{j \in S} q_{i j}(t) f(j)=\sum_{j \neq i} q_{i j}(t)(f(j)-f(i))
$$

Definição A.25. (Gerador)

A matriz $Q(t), t \geq 0$, é um gerador infinitesimal (ou simplesmente um gerador) da cadeia de markov $X=\left(X_{t}\right)_{t \geq 0}$ se ela satisfaz a $q$-Propriedade e para toda função $f$ de valor real definida em $S$

$$
f\left(X_{t}\right)-\int_{0}^{t} Q(s) f(.)\left(X_{s}\right) d s
$$

é um martingal.

Lema A.26. ( Yin e Zhang (1998))

Se $S=\{1,2, \ldots, m\}$. Então o processo $X_{t} \in S, t \geq 0$ é uma Cadeia de Markov gerada por $Q(t)$ se somente se

$$
\left(I\left(X_{t}=1\right), \ldots, I\left(X_{t}=m\right)\right)-\int_{0}^{t}\left(I\left(X_{s}=1\right), \ldots, I\left(X_{s}=m\right)\right) Q(s) d s
$$

é um martingal.

\section{A.2 Definições em Confiabilidade}

Nesta seção daremos algumas definições relativas à teoria da confiabilidade as quais usaremos ao longo deste trabalho. 
Consideremos um sistema composto de $n$ componentes, $C_{1}, \ldots, C_{n}$ com tempos de vida aleatórios $S_{i}, i=1, \ldots, n$. Seja $\Phi:\{0,1\}^{n} \rightarrow\{0,1\}$ a função de estrutura do sistema, que assumimos ser uma função monótona. Os possíveis estados dos componentes e do sistema serão indicados por "1"e "0" que representa o funcionamento e a falha do sistema (componente) respectivamente. A função $\Phi_{t}=\Phi\left(\mathbf{X}_{t}\right)$ descreve o estado do sistema no instante $t$, onde $\mathbf{X}_{t}=\left(X_{t}(1), \ldots, X_{t}(n)\right)$ e $X_{t}(i)$ denota a função indicadora

$$
X_{t}(i)=I\left(S_{i}>t\right)= \begin{cases}1, & \text { se } S_{i}>t \\ 0 & \text { se } S_{i} \leq t\end{cases}
$$

o qual é 1 , se o componente $i$ esta funcionando até o instante $t$, e 0 caso contrário. O tempo de vida do sistema, o qual denotaremos por $T$ é dado por $T=$ inf $\{t \in$ $\left.\mathbb{R}_{+}: \Phi_{t}=0\right\}$. Usaremos a seguinte notação para descrever o tempo de vida $T$ de um sistema com função estrutura $\Phi$ composto de tempos vidas $S_{i}, i=1, \ldots, n$ por:

$$
T=\Phi(\mathbf{S})=\Phi\left(S_{1}, \ldots, S_{n}\right)
$$

\section{Exemplo A.27.}

Considere um sistema composto de três componentes, o qual está funcionando se o componente 1 e pelo menos um dos componentes 2 ou 3 estão funcionando (veja figura abaixo):

Neste exemplo $\Phi_{t}=X_{t}(1)\left\{1-\left(1-X_{t}(2)\right)\left(1-X_{t}(3)\right)\right\}$ é facilmente obtido com $T=\inf \left\{t \in \mathbb{R}_{+}: \Phi_{t}=0\right\}=S_{1} \wedge\left(S_{2} \vee S_{3}\right)$, onde usamos a notação $a \wedge b$ e $a \vee b$ para denotar $\min \{a, b\}$ e $\max \{a, b\}$, respectivamente.

Vejamos agora algumas definições: 


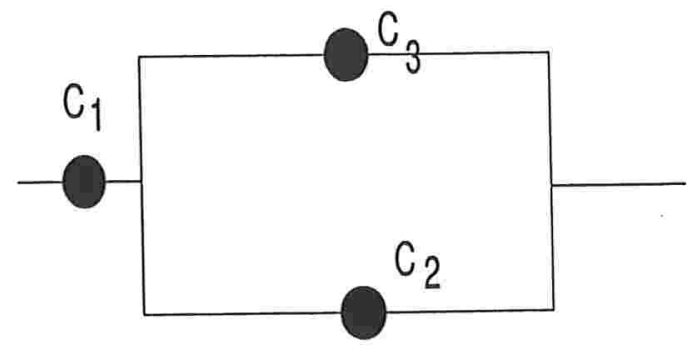

Figura A.1: $\Phi_{t}=X_{t}(1)\left\{1-\left(1-X_{t}(2)\right)\left(1-X_{t}(3)\right)\right\}$

\section{Definição A.28. (Sistemas Monótonos)}

Um sistema é dito monótono se sua função estrutura $\Phi$ é não decrescente em todo seu argumento com, $\Phi(\mathbf{0})=0$ e $\Phi(\mathbf{1})=1$.

Os sistemas considerados neste trabalho são denominados sistemas coerentes que constituem uma subclasse dos sistemas monótonos. Antes de definirmos o que é um sistema coerente precisamos de algumas notações.

$\mathrm{O}$ vetor $\left({ }_{i}, \mathrm{x}\right)$ denota um vetor estado, onde o estado do $i$-ésimo componente é igual a 1 ou $0 ;\left(1_{i}, \mathbf{x}\right)$ denota um vetor estado onde o estado do $i$-ésimo componente é igual a 1 e $\left(0_{i}, \mathbf{x}\right)$ denota um vetor estado onde o estado do $i$-ésimo componente é igual a 0 ; o estado do componente $j, j \neq i$ é igual a $x_{j}$. Se queremos especificar o estado de algums componentes, digamos $i \in J(J \subset\{1,2, \ldots, n\})$, usamos a notação $\left({ }_{J}, \mathbf{x}\right)$. Por exemplo, $\left(0_{J}, \mathbf{x}\right)$ denota o vetor estado onde os estados dos componentes em $J$ são todos 0 e o estado dos componentes $i, i \notin J$ iguais a $x_{i}$.

\section{Definição A.29. (Sistemas Coerentes)}

Um sistema $\Phi$ é dito coerente se é monótono e todos os seus componentes são relevantes, i.e., existe pelo menos um vetor $\left({ }_{\cdot i}, \mathbf{x}\right)$ tal que $\Phi\left(0_{i}, \mathbf{x}\right)=0$ e $\Phi\left(1_{i}, \mathbf{x}\right)=1$. 
Definição A.30. (Conjuntos de Corte Minimais)

Um conjunto de corte $K$ é um conjunto de componentes que falhando causa a falha do sistema, i.e., $\Phi\left(0_{K}, 1\right)=0$. Um conjunto de corte é minimal se ele não pode ser reduzido sem perder esta propriedade.

Definição A.31. (Conjuntos de Caminhos Minimais)

Um conjunto de caminho $S$ é um conjunto de componentes que se estão funcionando então o sistema funciona, i.e., $\Phi\left(1_{S}, 0\right)=1$. Um conjunto de caminho é minimal se ele não pode ser reduzido sem perder esta propriedade.

Teorema A.32. (Estrutura série-paralelo e paralelo-série)

Se $\Phi$ é uma estrutura coerente de $n$ componentes com conjuntos de cortes minimais $K_{1}, \ldots, K_{k}$ e conjunto de caminhos minimais $P_{1}, \ldots, P_{s}$, então:

$$
\begin{aligned}
\Phi\left(\mathbf{X}_{t}\right) & =\min _{1 \leq j \leq k} \max _{i \in K_{j}} X_{t}(i) \\
& =\max _{1 \leq j \leq s} \min _{i \in P_{j}} X_{t}(i) .
\end{aligned}
$$

Ou de forma equivalente, o tempo de vida do sistema $T$ pode ser escrito por:

$$
\begin{aligned}
T & =\min _{1 \leq j \leq k} \max _{i \in K_{j}} S_{i} \\
& =\max _{1 \leq j \leq s} \min _{i \in P_{j}} S_{i} .
\end{aligned}
$$

\section{Sistemas Monótonos em Multiestados}

Consideramos um sistema formado por $n$ componentes, numerados de 1 a $n$. Assim como no caso binário, $x_{i}$ representa o estado do componente $i, i=1, \ldots, n$, com 
valores no conjunto de estados $S_{i}=\left\{0, \ldots, M_{i}\right\}$,

$$
x_{i 0}, x_{i 1}, \ldots, x_{i M_{i}},\left(x_{i 0}<x_{i 1}<\ldots<x_{i M_{i}}\right) .
$$

Os estados $x_{i j}$ representa, por exemplo, os diferentes níveis de funcionamento, do pior, $x_{i 0}$, para o melhor, $x_{i M_{i}}$.

Similarmente, $\Phi=\Phi(\mathbf{x})$ denota os estados (níveis) do sistema. Denotaremos os vários valores que $\Phi$ pode tomar por

$$
\Phi_{0}, \Phi_{1}, \ldots, \Phi_{M},\left(\Phi_{0}<\Phi_{1}<\ldots<\Phi_{M}\right) .
$$

Se $M_{i}=1, i=1, \ldots, n$, e $M=1$ então o modelo é binário.

Definição A.33. (Sistemas Monótonos em Multiestados)

Um sistema em multiestados é dito monótono se:

(i) sua função estrutura $\Phi$ é não decrescente em todo seu argumento, $e$

(ii) $\Phi\left(x_{10}, \ldots, x_{n 0}\right)=\Phi_{0}$ e $\Phi\left(x_{1 M_{1}}, \ldots, x_{n M_{n}}\right)=\Phi_{M}$. 


\section{Referências Bibliográficas}

[1] E. Arjas: The failure and hazard processes in multivariate reliability system, Mathematics of Operations Research, 6 (1981), 551-562.

[2] E. Arjas: Survival Models and Martingale Dynamics, Scandinavian Journal Statistics, 16 (1989) 177-225.

[3] E. Arjas e F. Norros: Life lenghts and association: a dynamic approach, Mathematics of Operations Research, 9(1)(1984), 151-158.

[4] T. Aven e U. Jensen: Stochatic Models in Reliability, Springer-Verlag, New York (1999).

[5] T. Aven: On the computation of certain measures of importance of system components, Microel. Reliab. 26 (1986), 279-281.

[6] R. E. Barlow and F. Proschan: Importance of system components and fault tree events, Stochastic Processes and their Applications, 3 (1975), 153-173.

[7] R. E. Barlow and F. Proschan : Statistical Theory of Reliability and Life Testing: Probability models, Hold, Rinehart and Wiston, Silver Spring, MD, (1981). 
[8] Z. W. Birnbaum : On the importance of different components in a multicomponent system, In Multivariate Analysis II, P.R. Krishnaiah, Academic Press, New York (1969).

[9] P.J. Boland, E. El-Neweihi: Measures of component importance in reliability Theory, Computation of Operations Research, 22,(4)(1985), 455-463.

[10] P. Bremaud: Point Processes and Queues, Springer-Verlag, New York (1982). J O CAPÍ́ $<0$

[11] V.C. Bueno: Component importance in a random environment, Statistics and Probability Letters, 48 (2000), 173-179.

[12] V.C. Bueno and J.E. Menezes: Pattern's Reliability Importance Under Dependence Condition And Different Information Levels, RT-USP/MAE, $n^{o}-3$, São Paulo (2004).

[13] K.L. Chung: A Course in Probability Theory, Harcourt, Brace and World (1968).

[14] C. Dellacherie e P.A. Meyer: Probabilities and Potential, B. North-Holland, Amsterdam (1980).

[15] S. Iyer : The Barlow-Proschan importance and its generalizations with dependent components, Stochastic Processes and their Applications, 42 (1992), 353-359.

[16] J. Jacod: Multivariate point processes: predictable projection, Radon-Nikodym derivatives, representation of martingales, Wahrscheinlichkeitstheoric Verw. Gabiete, 31 (1975), 235-253.

[17] F. Jensen e N. Peterson : Burn-in, Wiley-Interscience Pubrication, (1982). 
[18] U. Jensen : A general replacement model, ZOR-Methods and Models of Operation Research, 34 (1990), 423-439.

[19] U. Jensen : Monotone stopping rules for stochastic processes in a semimartingale representation with applications, Optimization, 20 (1989), 837-852.

[20] J.E. Menezes: A Burn-in Model, RT-USP/MAE, nº12, São Paulo (2004).

[21] P. A. Meyer : Demostration simplifiér dûu théoréme de Knight, Seminaire de Probabilite, V, Springer Verlag, Berlin (1971).

[22] B. Natvig : New light on measure of importance of systems components, Scandinavian Journal of Statistics, 12 (1985), 43-54.

[23] I. Norros: A compensator representation of multivariate lifelength distributions, with applications, Scandinavian Journal of Statistics, 13 (1986a),99-112.

[24] I. Norros : Notes on Natvig's measure of importance of system's components, Journal of Applied Probability, 23 (1986b),736-747.

[25] M. Xie : A note on the Natvig measure, Scandinavian Journal of Statistics, 15 (1988), 211-214.

[26] G. George Yin and Q. Zhang: Continuous-Time Markov Chains and Applications, Springer, New York (1998). 